\begin{tabular}{|c|c|c|c|c|c|c|}
\hline \multirow{4}{*}{ Impact Factor: } & ISRA (India) & $=3.117$ & SIS (USA) & $=0.912$ & ICV (Poland) & $=6.630$ \\
\hline & ISI (Dubai, UAE & $=0.829$ & РИНЦ (Russia & $=0.156$ & PIF (India) & $=1.940$ \\
\hline & GIF (Australia) & $=0.564$ & ESJI (KZ) & $=8.716$ & IBI (India) & $=4.260$ \\
\hline & JIF & $=1.500$ & SJIF (Morocco & $=5.667$ & OAJI (USA) & $=0.350$ \\
\hline
\end{tabular}

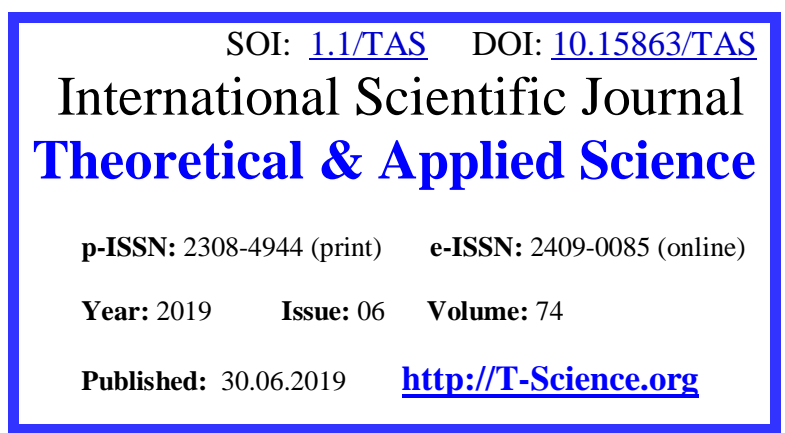

SECTION 31. Economic research, finance, innovation, risk management.

UDC 685: 54335.17
QR - Issue

QR - Article
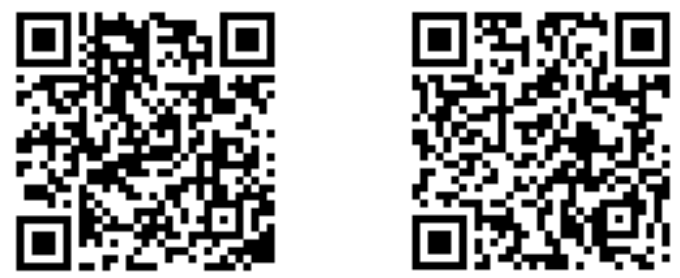

Artur Alexandrovich Blagorodov Institute of Entrepreneurship and Service sector (branch)

DSTU

bachelor, g. Shakhty

blaghorodov@list.ru

Dmitri Olegovich Bordukh

Institute of Entrepreneurship and Service sector (branch)

DSTU

bachelor, g. Shakhty

bordukh95@bk.ru

Angelina Vladimirovna Kopylova

Institute of Entrepreneurship and Service sector (branch)

DSTU

bachelor, g. Shakhty

angelinakopylova22121999@mail.ru

Vladimir Timofeevich Prokhorov

ISOP (f) DGTU

Doctor of technical sciences, professor, professor of the department "Designing, technology and design", Shakhty prohorov@sssu.ru

Igor Mikhailovich Maltsev Institute of Entrepreneurship and Sevice sector (branch) DSTU associate Professor, Ph. D.-m H., g. Shakhty

Natalia Vassilievna Tikhonova Kazan national research technological University Doctor of technical sciences, professor

Kazan, Republic of Tatarstan

\title{
EFFICIENCY CRITERIA, DESIGNED TO PRODUCE AN ADEQUATE RESPONSE TO A REASONABLE ASSESSMENT OF MANUFACTURING QUALITY ASSORTMENT OF CHILDREN'S FOOTWEAR
}

Abstract: The article presents the results of the survey of students, teachers and specialists - graduates who are now working at the enterprises of light industry SFD and North Caucasus Federal District regions, on the criteria for assessing the competitiveness of enterprises of light industry in the manufacture of products of light industry. In addition, the authors analyze the causes that trigger the production of products of light industry, not demanded by consumers because of its poor quality, due to its non-compliance with state standards and technical regulations. To import has all the prerequisites taken by customs barriers and entered sanctions, the main ways of development of competitive and marketable products, which are possible only if managers are professionally trained and politically responsible for the results of its operations, formulated the theoretical basis of the evaluation of the effectiveness of innovative technological solutions for the production of import of children's shoes at the enterprises of the Southern 


\begin{tabular}{|c|c|c|c|c|c|c|}
\hline \multirow{4}{*}{ Impact Factor: } & ISRA (India) & $=3.117$ & SIS (USA) & $=0.912$ & ICV (Poland) & $=6.630$ \\
\hline & ISI (Dubai, UAI & $=0.829$ & РИНЦ (Russia & $=0.156$ & PIF (India) & $=1.940$ \\
\hline & GIF (Australia) & $=0.564$ & ESJI (KZ) & $=8.716$ & IBI (India) & $=4.260$ \\
\hline & JIF & $=1.500$ & SJIF (Morocco & $=5.667$ & OAJI (USA) & $=0.350$ \\
\hline
\end{tabular}

Federal District and the North Caucasian Federal District regions. Representation of the correlation of the range of products and innovative solutions adopted for the production of these products.

Key words: software, questionnaire, assessment of the validity, relevance, respondents, expertise, product range, children's shoes, coefficient of concordance, the reliability criteria, import substitution, innovative technological solutions, assortment, competitiveness, demand, comfort, product range of children's shoes.

Language: English

Citation: Blagorodov, A. A., Bordukh, D. O., Kopylova, A. V., Prokhorov, V. T., Maltsev, I. M., \& Tikhonova, N. V. (2019). Efficiency criteria, designed to produce an adequate response to a reasonable assessment of manufacturing quality assortment of children's footwear. ISJ Theoretical \& Applied Science, 06 (74), 701-734

Soi: http://s-o-i.org/1.1/TAS-06-74-86 Doi: crossef https://dx.doi.org/10.15863/TAS.2019.06.74.86

\section{Introduction}

The absence in the Southern Federal District and the North Caucasian Federal District shoe enterprises and filling markets with domestic products not only provokes a deficit, but significantly impairs social status living in these regions, as for most people it was the only source of income, as these enterprises are town-forming and provides all the infrastructure of life population, causing not only employment, which in itself is very important, but also ensures the supply to these regions means to resolve all their social problems [1-2].

Hope regional and federal branches of the fact that everything can be solved at the expense of the ruthless exploitation of natural resources, which is not only criminal but also a road to nowhere. And talk about what our domestic products are not in demand are groundless.
Out of this situation - well-developed product range and assortment overall policy framework of the unity of all branches of government, namely: municipal, regional and federal, in alliance with manufacturers to offer consumers their regions are not only in demand and competitive products, but most importantly - economically justifiable and ensuring sustainable businesses receive TEP, providing them a warning from bankruptcy and guaranteeing stability [3].

\section{Main part.}

Respondents were offered the questionnaire with the criteria of competitiveness assessment and the demand for children's shoes. The respondents were the children themselves, their parents, occasional buyers of children's shoes and footwear manufacturers for children (Tables 1 - 4).

\section{Table 1 Criteria for assessing the competitiveness of and demand for the eyes of children's shoes of the child}

\begin{tabular}{|l|l|l|}
\hline number & $\begin{array}{l}\text { The list of factors to evaluate the competitive capacity of enterprises SFD and North } \\
\text { Caucasus Federal District regions }\end{array}$ & \\
\hline one & Form forefoot & \\
\hline 2 & Quality children's shoes & \\
\hline 3 & The flexibility of children's shoes & \\
\hline four & Price of children's shoes & \\
\hline five & comfort & \\
\hline 6 & The level of service of parents and children in shops and malls & \\
\hline 7 & Colour & \\
\hline eight & The warranty period for children's shoes & \\
\hline 9 & The height of the heel portion elation -up to 40 mm & \\
\hline ten & Elation heel height of the shoe 40 mm -svyshe & \\
\hline eleven & Weight & \\
\hline 12 & Maintainability children's shoes its expediency & \\
\hline 13 & Materials for the bottom of shoes & \\
\hline 14 & Materials for uppers & \\
\hline 15 & Point of sale of footwear for children -Interior store or shopping center & \\
\hline sixteen & What types of children's shoes are preferred: Winter & \\
\hline 17 & autumn & \\
\hline 18 & spring & \\
\hline nineteen & summer & \\
\hline 20 & Durability fastening shoe bottom & \\
\hline 21 & Variety assortment of shoes for children in shops and malls & \\
\hline 22 & Compliance towards fashion & \\
\hline
\end{tabular}




\begin{tabular}{|c|c|c|c|c|c|c|}
\hline \multirow{4}{*}{ Impact Factor: } & ISRA (India) & $=3.117$ & SIS (USA) & $=0.912$ & ICV (Poland) & $=6.630$ \\
\hline & ISI (Dubai, UAE & $=0.829$ & РИНЦ (Russia & $=0.156$ & PIF (India) & $=1.940$ \\
\hline & GIF (Australia) & $=0.564$ & ESJI (KZ) & $=8.716$ & IBI (India) & $=4.260$ \\
\hline & JIF & $=1.500$ & SJIF (Morocce & $=5.667$ & OAJI (USA) & $=0.350$ \\
\hline
\end{tabular}

Table 2 - Criteria for assessing the competitiveness of and demand for children's shoes eyes of ordinary buyers

\begin{tabular}{|l|l|l|}
\hline number & $\begin{array}{l}\text { The list of factors to evaluate the competitive capacity of enterprises SFD and North } \\
\text { Caucasus Federal District regions }\end{array}$ & Rank \\
\hline one & Weight & \\
\hline 2 & Colour & \\
\hline 3 & Quality children's shoes & \\
\hline four & Functionality of children's shoes & \\
\hline five & Characterization of materials for shoe uppers & \\
\hline 6 & Compliance towards fashion & \\
\hline 7 & Price & \\
\hline eight & Characterization of materials for the bottom of shoes & \\
\hline 9 & comfort & \\
\hline ten & Elation heel height of the shoe - 40 mm & \\
\hline eleven & Elation heel height of the shoe 40 mm -svyshe & \\
\hline 12 & repairability & \\
\hline 13 & The warranty period for children's shoes & \\
\hline 14 & What types of children's shoes are preferred: Winter & \\
\hline 15 & autumn & \\
\hline sixteen & spring & \\
\hline 17 & summer & \\
\hline 18 & Durability fastening shoe bottom & \\
\hline
\end{tabular}

Table 3 - Criteria for assessing the competitiveness of and demand for the eyes of children's shoes companies footwear manufacturers SFD and North Caucasus Federal District regions

\begin{tabular}{|l|l|l|}
\hline \multirow{2}{*}{ number } & $\begin{array}{l}\text { The list of factors to evaluate the competitive capacity of enterprises SFD and North } \\
\text { Caucasus Federal District regions }\end{array}$ & Rank \\
\hline one & Weight & \\
\hline 2 & Colour & \\
\hline 3 & Quality children's shoes & \\
\hline four & Functionality of children's shoes & \\
\hline five & Characterization of materials for shoe uppers & \\
\hline 6 & Compliance towards fashion & \\
\hline 7 & Price & \\
\hline eight & Characterization of materials for the bottom of shoes & \\
\hline 9 & comfort & \\
\hline ten & Elation heel height of the shoe -40 mm & \\
\hline eleven & Elation heel height of the shoe 40 mm -svyshe & \\
\hline 12 & repairability & \\
\hline 13 & The warranty period for children's shoes & \\
\hline
\end{tabular}

Table 4 - Criteria for assessing the competitiveness of and demand for the eyes of children's shoes parents

\begin{tabular}{|l|l|l|}
\hline number & $\begin{array}{l}\text { The list of factors to evaluate the competitive capacity of enterprises SFD and North } \\
\text { Caucasus Federal District regions }\end{array}$ & Rank \\
\hline one & Weight & \\
\hline 2 & Colour & \\
\hline 3 & Quality children's shoes & $\begin{array}{l}\text { Color fastness applied to the shoe upper material to dry and wet abrasion and exposure } \\
\text { to sweat }\end{array}$ \\
\hline four & Flexibility & \\
\hline five & Durability fastening shoe bottom & \\
\hline 6 & Price & \\
\hline 7 & comfort & \\
\hline eight &
\end{tabular}




\begin{tabular}{|c|c|c|c|c|c|c|}
\hline \multirow{4}{*}{ Impact Factor: } & ISRA (India) & $=3.117$ & SIS (USA) & $=0.912$ & ICV (Poland) & $=6.630$ \\
\hline & ISI (Dubai, UAI & $=0.829$ & РИНЦ (Russia & $=0.156$ & PIF (India) & $=1.940$ \\
\hline & GIF (Australia) & $=0.564$ & ESJI (KZ) & $=8.716$ & IBI (India) & $=4.260$ \\
\hline & JIF & $=1.500$ & SJIF (Morocce & $=5.667$ & OAJI (USA) & $=0.350$ \\
\hline
\end{tabular}

\begin{tabular}{|l|l|l|}
\hline 9 & The deformation of the toe cap and backdrop & \\
\hline ten & repairability & \\
\hline eleven & The warranty period for children's shoes & \\
\hline
\end{tabular}

Characteristics of respondents the survey results - the children, their parents, ordinary consumers and manufacturers to assess the competitive potential of the Southern Federal District and the North Caucasian Federal District regions are shown in Tables 5-8.

After the 2008 crisis, the world has spent a lot of effort trying to get the economy back the same rapid growth as before. But the assumption that the problems caused by the crisis, at times, erroneous, and we should accept this and understand that the economy in the new " the post-post-crisis world" will work in new ways. About this in his article at Project Syndicate says the founder and president of the World Economic Forum in Davos, Klaus Schwab [].

K. Schwab identifies six characteristics of this new world.

-economic growth it will be slower, but potentially more stable than before the crisis;

- motive force growth will technological changes, and their impact will be more extensive and deeper than, for example, had the industrial revolution and its consequences in the XIX and XX century. "The current industrial revolution hit the economy like a tsunami, almost without warning, and with ruthless force", - K. Schwab warns;

-rate of change will be high due to the relationships, existing in the world today, the changes affect both the economic structure, government, security arrangements and the daily life of the people;

-Each standard should be revised every industry risks being inverted upside down. If you need an illustration, look at Uber, which changed not only the scope of commercial traffic, but also retail in general: "uberizing" goods and services - consumers enjoy, but do not own them;

-The 3D-printing industry will change, because the supply chain will have to disappear or transform. "Gone are the days when the big fish ate the small. Fast fish will dominate the post-crisis world, slow dies", - said K. Schwab;

-economic growth will not push capital and natural resources, and human imagination and innovation.

According to the economist, in spite of the difficulties that would entail new round of technological progress, its total impact will be positive.

The decline in production of children's shoes, like shoes other age and gender groups, linked primarily to the lack of high-quality and affordable hides. Reducing subsidies to agriculture, as well as reducing the number of cattle in farms of Kalmykia, Krasnodar and Stavropol territories and weak base for the processing of hides, in turn, leads to a reduction in the rate of growth of production and hard chrometanned leather.

As a consequence, the domestic market, as the SFD and North Caucasus Federal District and Russia as a whole, began to fill brought from abroad, children's shoes, which, with rare exceptions, does not even have a certificate of quality, and now the children are forced to wear shoes made of artificial leather.

Thus, the restoration of volumes of production of children's shoes is rather urgent task facing the shoemaker, and has great social and economic importance to the Southern Federal District and the North Caucasian Federal District.

For the revival of the production of children's shoes in the Southern and North Caucasus Federal District, in the first place, the necessary organizational and financial support shoe enterprises in the Russian Government, regional and local authorities, in the form of lower VAT, grants a favorable interest rate with a grace period of 3 years, support in providing quality and affordable shoe materials.

Despite the fact that the industry produces children's shoes is undergoing a painful process of restructuring, the elimination of outdated and inefficient capacity, unfair competition from imports, and often simply in the fight against smuggling, today in light industries form a healthy core of several dozen major companies and hundreds of small and medium firms which have found their place in the market, began to produce marketable products.

Strategy of development of light industry up to 2025, the Russian government put in front of the light industry, real, achievable goals, namely []:

- make an inventory and assessment of the technical level of production capacity, which is still preserved. This is necessary in order to prioritize and forecast production update.

- to cancel a three-year import duties and VAT on imported on import technological equipment for textile and light industry, which is not produced in Russia.

- introduce differentiated taxation of fixed assets according to the terms of their operation, thereby stimulate the renewal of their active part.

- exemption from taxation that part of the profit, which is directed to the modernization of production.

- the creation of the country's industry-leasing company, perhaps with the participation of the state capital, in the likeness of "Agropromlizing"

- given that the depreciation of fixed assets of companies have little or no collateral value, to ensure that the federal bodies of executive power and subjects of the Russian Federation acts as a guarantor of realization of the most significant engineering projects. 


\begin{tabular}{|c|c|c|c|c|c|c|}
\hline \multirow{4}{*}{ Impact Factor: } & ISRA (India) & $=3.117$ & SIS (USA) & $=0.912$ & ICV (Poland) & $=6.630$ \\
\hline & ISI (Dubai, UAI & $=0.829$ & РИНЦ (Russia & $=0.156$ & PIF (India) & $=1.940$ \\
\hline & GIF (Australia) & $=0.564$ & ESJI (KZ) & $=8.716$ & IBI (India) & $=4.260$ \\
\hline & JIF & $=1.500$ & SJIF (Morocco & $=5.667$ & OAJI (USA) & $=0.350$ \\
\hline
\end{tabular}

Next problem - the creation of conditions for shoe enterprises of fair competition, excluding the huge scale of illegal imports of cheap low-quality products from abroad. This requires an increase in customs duty on imported shoes.

It is impossible not to recognize that prevailing in the footwear industry SFD and North Caucasus Federal District the situation, not least of all - the result of the inability of many executives shoe enterprises SFD and North Caucasus Federal District to quickly adapt to new requirements imposed by the market, to competition arose, and with Russian and foreign manufacturers.

In developing the range of children's shoes need to consider the factors shaping consumer demand: the main line fashion trends, and the economic, social and climatic specifics of regions of the Southern Federal District and the North Caucasian Federal District.

The combination of software competence assessment survey results with respondents representing different layers of the Southern Federal District and the North Caucasian Federal District regions of the population and develop innovative technological processes for the production of the entire product range of footwear using the dimensionless efficiency factor ( $\mathrm{K}$ eff) form the basis of the producers for making guaranteed solution for justification received solutions in the formation of new industries SFD and North Caucasus Federal District regions in order to fill the markets of these regions import-substituting products with the creation of new jobs, especially in small and medium-sized cities, where there is currently a tense situation with the provision of jobs. This software product allows content producers to federal, regional and municipal branches of government to be confident in making the validity of them taken on the organization of new productions in small and medium-sized cities, turning them into a town-forming and protecting the populations of these cities from the need to migrate in search of a survival of solving many of the social programs of the population of these cities, and keep them from destruction turning some regions of overpopulation due to migration, and others from the destruction that does not contribute to prosperity in general, suitable to the life of the regions. It is only important to make timely decisions and to form such production which is demanded by the population of these cities and in parallel to solve at all levels of government, all social problems of the population in these regions by opening a school, childcare, sports and health centers, to the population of these regions was really comfortable and not to provoke them to migrate here only it were not too late, as it did with the coal regions of the justification for our conclusions about the effectiveness of the software is due to the positive assessment of the investors and managers of enterprises of light industry, such an opinion was made possible by participation by respondents in surveys on topical issues, to obtain an objective assessment and recommendations within range policy for the production of peeled Bova and competitive products, including children's demand for which has for many years is not satisfied, and the use of imported products provokes their formation abnormalities, allergies and other chronic diseases, because this product does not meet the requirements of GOST and technical regulations. Thus, there are all prerequisites for the implementation of our proposals for the formation of efficient production with the production of marketable and competitive products, this requires good will of all parties concerned.

Analysis of the results of the survey respondents on the criteria for assessing the competitiveness of and demand for children's shoes are given in Tables $5-8$ with the participation of children, their parents, consumers and manufacturers, have confirmed the expected result, namely, the fact that the competence of the participants in the survey differ significantly and do not allow one to take the only right decision for themselves the criteria for assessing the competitiveness of and demand for all kinds of shoes for the product range, allows to make a decision about the relevance and competitive capacity of enterprises in the manufacture of footwear for children.

Designed by the authors of the software for the distribution of all the participants in the survey on the extent of their competence on the mentioned process using the concordance coefficient has confirmed its high efficiency for all participants in the survey.

Less expertise have natural children, because they have in completing the questionnaire in the first place, such criteria were defined as the mass of the comfort variety of assortment of shoes in a store, service levels, and not those who warn of the child and the parents of the possible formation of their child pathological foot abnormalities. The results of the survey of parents do not have a high competence of the criteria for assessing the competitiveness of and demand for children's shoes, but they are united by the desire to see in-store quality footwear, in which they put the following understanding: comfort, weight, price and warranty period, although this factor is a rapidly growing child's foot less significant. But maybe this is the voice of the parents in a family where three or more children with no significant difference in age. Surprised by the results of a survey of buyers who consciously choose to shop with children's footwear, and the desire to buy a shoe, which is necessary for their child, it is possible to speak of a conscious choice. Confirmation of our assumptions is the fact that customers - both men and women - were unanimous in saying that the children's shoes should be comfortable, easy and affordable. But on the other hand it confirmed the fact that having a lack of competence and consistency in the evaluation criteria of competitiveness and demand for children's shoes, while they assessed the available in-store product 


\begin{tabular}{|c|c|c|c|c|c|c|}
\hline \multirow{4}{*}{ Impact Factor: } & ISRA (India) & $=3.117$ & SIS (USA) & $=0.912$ & ICV (Poland) & $=6.630$ \\
\hline & ISI (Dubai, UAE & $=0.829$ & РИНЦ (Russia & $=0.156$ & PIF (India) & $=1.940$ \\
\hline & GIF (Australia) & $=0.564$ & ESJI (KZ) & $=8.716$ & IBI (India) & $=4.260$ \\
\hline & JIF & $=1.500$ & SJIF (Morocce & $=5.667$ & OAJI (USA) & $=0.350$ \\
\hline
\end{tabular}

range of footwear exclusively with consumer products. And almost no attention to the criteria that would protect their children from the stack formation abnormalities sad doctors - orthopedists in children's institutions is not enough pay attention to outreach to parents, provoking them to a more informed decision about buying shoes to your child, preferring a shoe that all and only all criteria guarantees a child a warning of his foot from the formation of pathological deviations. Were only competent answers to the questionnaire on the criteria for assessing the competitiveness of and demand for children's footwear manufacturing representatives. Their criteria quality children's shoes, children's shoes functionality, comfort, weight, material properties for the top and bottom of footwear, confirms their understanding of the importance of compliance with state standards and technical regulations governing the conditions for the execution of which ensures children are not only comfortable conditions of the foot, but also protects it from the formation of pathological abnormalities. The good news is the fact that their competence has a high level of consistency with other experts, as evidenced by the concordance coefficient $\mathrm{W}$ value close to unity. That is, the parents have a good reason to buy shoes made from the likes of knowing that their children's foot is not in danger.

Table 5 - Results competency assessment of children who participated in the survey on the assessment competitivenesses and demand for children's shoes

\begin{tabular}{|c|c|c|c|c|c|c|c|c|c|c|c|c|c|c|c|c|c|c|c|c|c|c|c|c|c|}
\hline $\begin{array}{c}\text { Экспе } \\
\text { рты }\end{array}$ & $\begin{array}{l}\text { Факто } \\
\text { ры }\end{array}$ & 1 & 2 & 3 & 4 & 5 & 6 & 7 & 8 & 9 & $\begin{array}{l}1 \\
\mathbf{0}\end{array}$ & $\begin{array}{l}1 \\
1\end{array}$ & $\begin{array}{l}1 \\
2\end{array}$ & $\begin{array}{l}1 \\
3\end{array}$ & $\begin{array}{l}1 \\
4\end{array}$ & $\begin{array}{l}1 \\
5\end{array}$ & $\begin{array}{l}1 \\
6\end{array}$ & $\begin{array}{l}1 \\
7\end{array}$ & $\begin{array}{l}1 \\
8\end{array}$ & $\begin{array}{l}1 \\
9\end{array}$ & $\begin{array}{l}2 \\
0\end{array}$ & $\begin{array}{l}2 \\
1\end{array}$ & $\begin{array}{l}2 \\
2\end{array}$ & $\begin{array}{l}2 \\
3\end{array}$ & 24 \\
\hline 2-й & & 3 & 2 & $\begin{array}{l}1 \\
4 \\
\end{array}$ & $\begin{array}{l}1 \\
3 \\
\end{array}$ & 8 & 9 & $\begin{array}{l}1 \\
5 \\
\end{array}$ & 5 & $\begin{array}{l}1 \\
6 \\
\end{array}$ & $\begin{array}{l}1 \\
0 \\
\end{array}$ & $\begin{array}{l}1 \\
2 \\
\end{array}$ & $\begin{array}{l}1 \\
7 \\
\end{array}$ & 1 & $\begin{array}{l}1 \\
8 \\
\end{array}$ & 4 & $\begin{array}{l}1 \\
9 \\
\end{array}$ & 6 & $\begin{array}{l}1 \\
0 \\
\end{array}$ & $\begin{array}{l}2 \\
0 \\
\end{array}$ & $\begin{array}{l}2 \\
1 \\
\end{array}$ & $\begin{array}{l}1 \\
1 \\
\end{array}$ & 7 & & $\begin{array}{l}0,661 \\
769 \\
\end{array}$ \\
\hline 3-й & & 8 & $\begin{array}{l}1 \\
6 \\
\end{array}$ & $\begin{array}{l}2 \\
1 \\
\end{array}$ & 5 & 2 & $\begin{array}{l}1 \\
0 \\
\end{array}$ & 6 & 7 & $\begin{array}{l}1 \\
1 \\
\end{array}$ & $\begin{array}{l}1 \\
7 \\
\end{array}$ & $\begin{array}{l}1 \\
2 \\
\end{array}$ & $\begin{array}{l}1 \\
4 \\
\end{array}$ & 1 & $\begin{array}{l}2 \\
0 \\
\end{array}$ & 3 & $\begin{array}{l}1 \\
3 \\
\end{array}$ & $\begin{array}{l}1 \\
5 \\
\end{array}$ & $\begin{array}{l}1 \\
7 \\
\end{array}$ & $\begin{array}{l}1 \\
9 \\
\end{array}$ & $\begin{array}{l}1 \\
8 \\
\end{array}$ & 4 & 9 & & $\begin{array}{l}0,668 \\
55\end{array}$ \\
\hline 4-й & & $\begin{array}{l}1 \\
0\end{array}$ & $\begin{array}{l}1 \\
3\end{array}$ & $\begin{array}{l}2 \\
1\end{array}$ & $\begin{array}{l}1 \\
4\end{array}$ & 2 & 6 & $\begin{array}{l}1 \\
1\end{array}$ & 4 & 5 & 7 & 9 & $\begin{array}{l}1 \\
9 \\
\end{array}$ & 1 & $\begin{array}{l}1 \\
8\end{array}$ & 3 & $\begin{array}{l}1 \\
5\end{array}$ & $\begin{array}{l}1 \\
6 \\
\end{array}$ & 7 & $\begin{array}{l}1 \\
7 \\
\end{array}$ & $\begin{array}{l}2 \\
0\end{array}$ & 8 & $\begin{array}{l}1 \\
2\end{array}$ & & $\begin{array}{l}0,555 \\
807\end{array}$ \\
\hline 5-й & & $\begin{array}{l}1 \\
5\end{array}$ & 2 & $\begin{array}{l}1 \\
6\end{array}$ & $\begin{array}{l}1 \\
4\end{array}$ & $\begin{array}{l}1 \\
7\end{array}$ & 3 & 2 & 5 & 6 & $\begin{array}{l}1 \\
3 \\
\end{array}$ & 7 & $\begin{array}{l}1 \\
0\end{array}$ & 1 & 8 & $\begin{array}{l}1 \\
8\end{array}$ & $\begin{array}{l}2 \\
1\end{array}$ & 9 & $\begin{array}{l}2 \\
0\end{array}$ & $\begin{array}{l}1 \\
9 \\
\end{array}$ & $\begin{array}{l}1 \\
1\end{array}$ & 4 & $\begin{array}{l}1 \\
2\end{array}$ & & $\begin{array}{l}0,496 \\
609\end{array}$ \\
\hline 6-й & & 1 & 2 & $\begin{array}{l}1 \\
0\end{array}$ & $\begin{array}{l}1 \\
2\end{array}$ & 7 & $\begin{array}{l}1 \\
3 \\
\end{array}$ & $\begin{array}{l}1 \\
1\end{array}$ & 3 & $\begin{array}{l}1 \\
4 \\
\end{array}$ & $\begin{array}{l}1 \\
5 \\
\end{array}$ & 8 & $\begin{array}{l}1 \\
6 \\
\end{array}$ & $\begin{array}{l}1 \\
7 \\
\end{array}$ & $\begin{array}{l}2 \\
1 \\
\end{array}$ & 4 & 9 & $\begin{array}{l}2 \\
0 \\
\end{array}$ & $\begin{array}{l}2 \\
2 \\
\end{array}$ & 5 & 6 & $\begin{array}{l}1 \\
9 \\
\end{array}$ & $\begin{array}{l}1 \\
8 \\
\end{array}$ & & $\begin{array}{l}0,733 \\
333\end{array}$ \\
\hline 7-й & & $\begin{array}{l}1 \\
2 \\
\end{array}$ & $\begin{array}{l}1 \\
1 \\
\end{array}$ & $\begin{array}{l}1 \\
4 \\
\end{array}$ & $\begin{array}{l}1 \\
6 \\
\end{array}$ & $\begin{array}{l}1 \\
0\end{array}$ & 9 & 2 & $\begin{array}{l}2 \\
0 \\
\end{array}$ & 8 & $\begin{array}{l}1 \\
9 \\
\end{array}$ & 7 & $\begin{array}{l}1 \\
8 \\
\end{array}$ & 1 & $\begin{array}{l}1 \\
3 \\
\end{array}$ & $\begin{array}{l}2 \\
2 \\
\end{array}$ & $\begin{array}{l}1 \\
5 \\
\end{array}$ & $\begin{array}{l}1 \\
7 \\
\end{array}$ & 6 & $\begin{array}{l}2 \\
1 \\
\end{array}$ & 5 & 3 & 4 & & $\begin{array}{l}0,417 \\
232 \\
\end{array}$ \\
\hline 8-й & & 2 & $\begin{array}{l}1 \\
9\end{array}$ & 9 & $\begin{array}{l}1 \\
2\end{array}$ & 8 & 3 & $\begin{array}{l}1 \\
1\end{array}$ & $\begin{array}{l}2 \\
0\end{array}$ & 4 & $\begin{array}{l}2 \\
2\end{array}$ & 7 & $\begin{array}{l}1 \\
3 \\
\end{array}$ & 5 & $\begin{array}{l}1 \\
7\end{array}$ & $\begin{array}{l}2 \\
1\end{array}$ & $\begin{array}{l}1 \\
0\end{array}$ & $\begin{array}{l}1 \\
4\end{array}$ & $\begin{array}{l}1 \\
8\end{array}$ & $\begin{array}{l}1 \\
6\end{array}$ & 1 & 6 & $\begin{array}{l}1 \\
5\end{array}$ & & $\begin{array}{l}0,471 \\
469\end{array}$ \\
\hline 9-й & & $\begin{array}{l}1 \\
0 \\
\end{array}$ & 4 & $\begin{array}{l}1 \\
8 \\
\end{array}$ & 3 & 8 & $\begin{array}{l}1 \\
9 \\
\end{array}$ & 9 & $\begin{array}{l}1 \\
4 \\
\end{array}$ & $\begin{array}{l}2 \\
1 \\
\end{array}$ & $\begin{array}{l}1 \\
5 \\
\end{array}$ & 5 & $\begin{array}{l}1 \\
7 \\
\end{array}$ & 1 & $\begin{array}{l}1 \\
2 \\
\end{array}$ & $\begin{array}{l}1 \\
1 \\
\end{array}$ & $\begin{array}{l}1 \\
6 \\
\end{array}$ & $\begin{array}{l}2 \\
0 \\
\end{array}$ & $\begin{array}{l}2 \\
2 \\
\end{array}$ & $\begin{array}{l}1 \\
3 \\
\end{array}$ & 6 & 2 & 7 & & $\begin{array}{l}0,597 \\
175\end{array}$ \\
\hline 10-й & & 6 & 7 & $\begin{array}{l}1 \\
7 \\
\end{array}$ & $\begin{array}{l}1 \\
8 \\
\end{array}$ & $\begin{array}{l}1 \\
6 \\
\end{array}$ & $\begin{array}{l}1 \\
4 \\
\end{array}$ & 5 & $\begin{array}{l}1 \\
9 \\
\end{array}$ & $\begin{array}{l}1 \\
3 \\
\end{array}$ & 8 & 4 & 9 & $\begin{array}{l}1 \\
0 \\
\end{array}$ & $\begin{array}{l}1 \\
1 \\
\end{array}$ & $\begin{array}{l}2 \\
2 \\
\end{array}$ & 3 & $\begin{array}{l}2 \\
1 \\
\end{array}$ & $\begin{array}{l}1 \\
2 \\
\end{array}$ & $\begin{array}{l}2 \\
0 \\
\end{array}$ & $\begin{array}{l}1 \\
5 \\
\end{array}$ & 1 & 2 & & $\begin{array}{l}0,381 \\
356 \\
\end{array}$ \\
\hline 11-й & & $\begin{array}{l}1 \\
0\end{array}$ & 5 & 4 & 9 & 3 & $\begin{array}{l}1 \\
2\end{array}$ & $\begin{array}{l}1 \\
1\end{array}$ & 8 & 1 & $\begin{array}{l}2 \\
2\end{array}$ & 2 & $\begin{array}{l}1 \\
3\end{array}$ & $\begin{array}{l}1 \\
4\end{array}$ & $\begin{array}{l}1 \\
6\end{array}$ & $\begin{array}{l}1 \\
7\end{array}$ & 6 & $\begin{array}{l}2 \\
0\end{array}$ & $\begin{array}{l}1 \\
8\end{array}$ & $\begin{array}{l}2 \\
1\end{array}$ & 7 & $\begin{array}{l}1 \\
9\end{array}$ & $\begin{array}{l}1 \\
5\end{array}$ & & $\begin{array}{l}0,674 \\
576\end{array}$ \\
\hline 12-й & & 8 & 3 & 9 & $\begin{array}{l}1 \\
3 \\
\end{array}$ & 2 & $\begin{array}{l}2 \\
2 \\
\end{array}$ & $\begin{array}{l}1 \\
4 \\
\end{array}$ & $\begin{array}{l}1 \\
1 \\
\end{array}$ & $\begin{array}{l}1 \\
5 \\
\end{array}$ & $\begin{array}{l}1 \\
9 \\
\end{array}$ & 4 & $\begin{array}{l}1 \\
7 \\
\end{array}$ & 6 & $\begin{array}{l}1 \\
6 \\
\end{array}$ & $\begin{array}{l}2 \\
0 \\
\end{array}$ & $\begin{array}{l}1 \\
0 \\
\end{array}$ & $\begin{array}{l}1 \\
8 \\
\end{array}$ & $\begin{array}{l}2 \\
1 \\
\end{array}$ & $\begin{array}{l}1 \\
2 \\
\end{array}$ & 1 & 5 & 7 & & $\begin{array}{l}0,551 \\
13 \\
\end{array}$ \\
\hline 13-й & & 4 & 1 & 9 & 6 & $\begin{array}{l}1 \\
3 \\
\end{array}$ & $\begin{array}{l}1 \\
5 \\
\end{array}$ & 3 & $\begin{array}{l}1 \\
9 \\
\end{array}$ & $\begin{array}{l}1 \\
4 \\
\end{array}$ & 8 & $\begin{array}{l}1 \\
8 \\
\end{array}$ & $\begin{array}{l}2 \\
0 \\
\end{array}$ & $\begin{array}{l}1 \\
7 \\
\end{array}$ & $\begin{array}{l}2 \\
1 \\
\end{array}$ & 5 & $\begin{array}{l}1 \\
6 \\
\end{array}$ & $\begin{array}{l}1 \\
0 \\
\end{array}$ & 2 & $\begin{array}{l}2 \\
2 \\
\end{array}$ & $\begin{array}{l}1 \\
2 \\
\end{array}$ & 7 & $\begin{array}{l}1 \\
1 \\
\end{array}$ & & $\begin{array}{l}0,595 \\
48 \\
\end{array}$ \\
\hline 14-й & & $\begin{array}{l}1 \\
3\end{array}$ & $\begin{array}{l}1 \\
4\end{array}$ & $\begin{array}{l}1 \\
0\end{array}$ & 3 & 1 & 2 & $\begin{array}{l}1 \\
6\end{array}$ & $\begin{array}{l}1 \\
5\end{array}$ & $\begin{array}{l}2 \\
0\end{array}$ & 5 & $\begin{array}{l}2 \\
1\end{array}$ & $\begin{array}{l}1 \\
7\end{array}$ & 4 & $\begin{array}{l}1 \\
1\end{array}$ & $\begin{array}{l}1 \\
9 \\
\end{array}$ & 7 & $\begin{array}{l}1 \\
8\end{array}$ & 6 & $\begin{array}{l}2 \\
2\end{array}$ & 9 & $\begin{array}{l}1 \\
2\end{array}$ & 8 & & $\begin{array}{l}0,564 \\
689\end{array}$ \\
\hline 15-й & & 7 & $\begin{array}{l}1 \\
4\end{array}$ & 3 & $\begin{array}{l}1 \\
1\end{array}$ & $\begin{array}{l}1 \\
7\end{array}$ & $\begin{array}{l}1 \\
9\end{array}$ & 4 & $\begin{array}{l}1 \\
2\end{array}$ & 9 & $\begin{array}{l}2 \\
1\end{array}$ & 1 & $\begin{array}{l}1 \\
8 \\
\end{array}$ & 5 & $\begin{array}{l}2 \\
0\end{array}$ & $\begin{array}{l}2 \\
2\end{array}$ & $\begin{array}{l}1 \\
5\end{array}$ & 8 & $\begin{array}{l}1 \\
6 \\
\end{array}$ & 2 & $\begin{array}{l}1 \\
3 \\
\end{array}$ & 6 & $\begin{array}{l}1 \\
0\end{array}$ & & $\begin{array}{l}0,431 \\
638\end{array}$ \\
\hline 16-й & & 2 & 3 & 5 & 6 & 8 & 4 & $\begin{array}{l}1 \\
0\end{array}$ & $\begin{array}{l}1 \\
5\end{array}$ & 7 & $\begin{array}{l}1 \\
1\end{array}$ & $\begin{array}{l}1 \\
8\end{array}$ & $\begin{array}{l}1 \\
6\end{array}$ & 1 & $\begin{array}{l}1 \\
2\end{array}$ & $\begin{array}{l}2 \\
1\end{array}$ & $\begin{array}{l}1 \\
9\end{array}$ & $\begin{array}{l}1 \\
3 \\
\end{array}$ & $\begin{array}{l}1 \\
4\end{array}$ & $\begin{array}{l}1 \\
7\end{array}$ & $\begin{array}{l}2 \\
2\end{array}$ & $\begin{array}{l}2 \\
0\end{array}$ & 9 & & $\begin{array}{l}0,707 \\
062\end{array}$ \\
\hline 17-й & & 6 & $\begin{array}{l}1 \\
5\end{array}$ & 7 & 8 & $\begin{array}{l}1 \\
1\end{array}$ & $\begin{array}{l}1 \\
0\end{array}$ & 9 & 1 & $\begin{array}{l}2 \\
1\end{array}$ & $\begin{array}{l}2 \\
0\end{array}$ & $\begin{array}{l}1 \\
6\end{array}$ & $\begin{array}{l}1 \\
7\end{array}$ & 2 & $\begin{array}{l}1 \\
2\end{array}$ & 3 & $\begin{array}{l}2 \\
2\end{array}$ & $\begin{array}{l}1 \\
9\end{array}$ & $\begin{array}{l}1 \\
3 \\
\end{array}$ & 4 & $\begin{array}{l}1 \\
8\end{array}$ & $\begin{array}{l}1 \\
4\end{array}$ & 5 & & $\begin{array}{l}0,779 \\
379\end{array}$ \\
\hline 18-й & & 3 & 1 & $\begin{array}{l}2 \\
2\end{array}$ & 6 & $\begin{array}{l}1 \\
9\end{array}$ & $\begin{array}{l}1 \\
3\end{array}$ & $\begin{array}{l}1 \\
4\end{array}$ & $\begin{array}{l}1 \\
1\end{array}$ & $\begin{array}{l}1 \\
7\end{array}$ & $\begin{array}{l}1 \\
8\end{array}$ & 2 & $\begin{array}{l}2 \\
1\end{array}$ & $\begin{array}{l}1 \\
2\end{array}$ & $\begin{array}{l}1 \\
6\end{array}$ & 4 & 5 & $\begin{array}{l}1 \\
0\end{array}$ & $\begin{array}{l}1 \\
5\end{array}$ & $\begin{array}{l}2 \\
0\end{array}$ & 7 & 8 & 9 & & $\begin{array}{l}0,560 \\
452\end{array}$ \\
\hline 19-й & & 2 & 3 & 6 & 7 & $\begin{array}{l}1 \\
2 \\
\end{array}$ & $\begin{array}{l}1 \\
1 \\
\end{array}$ & $\begin{array}{l}1 \\
7 \\
\end{array}$ & $\begin{array}{l}1 \\
3 \\
\end{array}$ & $\begin{array}{l}1 \\
8 \\
\end{array}$ & $\begin{array}{l}1 \\
6 \\
\end{array}$ & 1 & $\begin{array}{l}2 \\
0 \\
\end{array}$ & 5 & $\begin{array}{l}1 \\
4 \\
\end{array}$ & $\begin{array}{l}1 \\
9 \\
\end{array}$ & 8 & $\begin{array}{l}1 \\
5 \\
\end{array}$ & 9 & $\begin{array}{l}1 \\
0 \\
\end{array}$ & $\begin{array}{l}2 \\
2 \\
\end{array}$ & $\begin{array}{l}2 \\
1 \\
\end{array}$ & 4 & & $\begin{array}{l}0,599 \\
153 \\
\end{array}$ \\
\hline 20-й & & 2 & $\begin{array}{l}1 \\
2\end{array}$ & 8 & $\begin{array}{l}1 \\
1\end{array}$ & $\begin{array}{l}1 \\
4\end{array}$ & 7 & $\begin{array}{l}1 \\
5\end{array}$ & $\begin{array}{l}1 \\
0\end{array}$ & $\begin{array}{l}1 \\
7\end{array}$ & 9 & $\begin{array}{l}1 \\
6\end{array}$ & $\begin{array}{l}1 \\
8\end{array}$ & 1 & $\begin{array}{l}2 \\
0\end{array}$ & 5 & $\begin{array}{l}1 \\
9\end{array}$ & 4 & $\begin{array}{l}1 \\
3\end{array}$ & $\begin{array}{l}2 \\
2\end{array}$ & 6 & $\begin{array}{l}2 \\
1\end{array}$ & 3 & & $\begin{array}{l}0,723 \\
446\end{array}$ \\
\hline 21-й & & 1 & $\begin{array}{l}1 \\
4\end{array}$ & $\begin{array}{l}2 \\
1\end{array}$ & 9 & 8 & $\begin{array}{l}1 \\
5\end{array}$ & $\begin{array}{l}1 \\
6\end{array}$ & 7 & 5 & 6 & 4 & $\begin{array}{l}1 \\
8\end{array}$ & $\begin{array}{l}1 \\
9\end{array}$ & $\begin{array}{l}1 \\
7\end{array}$ & $\begin{array}{l}1 \\
0\end{array}$ & $\begin{array}{l}2 \\
0\end{array}$ & $\begin{array}{l}2 \\
2\end{array}$ & $\begin{array}{l}1 \\
1\end{array}$ & $\begin{array}{l}1 \\
2\end{array}$ & $\begin{array}{l}1 \\
3\end{array}$ & 2 & 3 & & $\begin{array}{l}0,613 \\
277\end{array}$ \\
\hline 22-й & & $\begin{array}{l}1 \\
0 \\
\end{array}$ & 1 & $\begin{array}{l}1 \\
8 \\
\end{array}$ & $\begin{array}{l}1 \\
1 \\
\end{array}$ & 5 & $\begin{array}{l}1 \\
2 \\
\end{array}$ & $\begin{array}{l}2 \\
0 \\
\end{array}$ & $\begin{array}{l}1 \\
9 \\
\end{array}$ & 6 & $\begin{array}{l}1 \\
5 \\
\end{array}$ & 7 & 8 & 2 & 9 & 4 & $\begin{array}{l}1 \\
3 \\
\end{array}$ & $\begin{array}{l}1 \\
7 \\
\end{array}$ & \begin{tabular}{|l}
1 \\
5 \\
\end{tabular} & $\begin{array}{l}1 \\
6 \\
\end{array}$ & $\begin{array}{l}2 \\
1 \\
\end{array}$ & 3 & $\begin{array}{l}1 \\
4 \\
\end{array}$ & & $\begin{array}{l}0,504 \\
662 \\
\end{array}$ \\
\hline 23-й & & 1 & 2 & 3 & 4 & 5 & 6 & 7 & 8 & 9 & $\begin{array}{l}1 \\
0 \\
\end{array}$ & $\begin{array}{l}1 \\
1 \\
\end{array}$ & $\begin{array}{l}1 \\
2 \\
\end{array}$ & $\begin{array}{l}1 \\
3 \\
\end{array}$ & $\begin{array}{l}1 \\
4 \\
\end{array}$ & $\begin{array}{l}1 \\
5 \\
\end{array}$ & $\begin{array}{l}1 \\
6 \\
\end{array}$ & $\begin{array}{l}1 \\
7 \\
\end{array}$ & $\begin{array}{l}1 \\
8 \\
\end{array}$ & $\begin{array}{l}1 \\
9 \\
\end{array}$ & $\begin{array}{l}2 \\
0 \\
\end{array}$ & $\begin{array}{l}2 \\
1 \\
\end{array}$ & $\begin{array}{l}2 \\
2 \\
\end{array}$ & & $\begin{array}{l}0,753 \\
672 \\
\end{array}$ \\
\hline
\end{tabular}




\begin{tabular}{|c|c|c|c|c|c|c|}
\hline \multirow{4}{*}{ Impact Factor: } & ISRA (India) & $=3.117$ & SIS (USA) & $=0.912$ & ICV (Poland) & $=6.630$ \\
\hline & ISI (Dubai, UAE & $=0.829$ & РИНЦ (Russia & $=0.156$ & PIF (India) & $=1.940$ \\
\hline & GIF (Australia) & $=0.564$ & ESJI (KZ) & $=8.716$ & IBI (India) & $=4.260$ \\
\hline & JIF & $=1.500$ & SJIF (Morocce & $=5.667$ & OAJI (USA) & $=0.350$ \\
\hline
\end{tabular}

\begin{tabular}{|c|c|c|c|c|c|c|c|c|c|c|c|c|c|c|c|c|c|c|c|c|c|c|c|}
\hline 24-й & 9 & 1 & $\begin{array}{l}1 \\
0 \\
\end{array}$ & $\begin{array}{l}1 \\
1 \\
\end{array}$ & 3 & 2 & $\begin{array}{l}1 \\
3 \\
\end{array}$ & $\begin{array}{l}1 \\
2 \\
\end{array}$ & $\begin{array}{l}1 \\
5 \\
\end{array}$ & $\begin{array}{l}1 \\
9 \\
\end{array}$ & 8 & 7 & $\begin{array}{l}1 \\
4 \\
\end{array}$ & $\begin{array}{l}1 \\
8 \\
\end{array}$ & $\begin{array}{l}2 \\
0 \\
\end{array}$ & 4 & $\begin{array}{l}1 \\
7 \\
\end{array}$ & $\begin{array}{l}2 \\
2 \\
\end{array}$ & $\begin{array}{l}1 \\
6 \\
\end{array}$ & $\begin{array}{l}2 \\
1\end{array}$ & 5 & 6 & \begin{tabular}{|l|l}
0,614 \\
124 \\
\end{tabular} \\
\hline 25-й & $\begin{array}{l}2 \\
0\end{array}$ & 4 & $\begin{array}{l}1 \\
1 \\
1\end{array}$ & $\begin{array}{l}1 \\
8 \\
\end{array}$ & 5 & 6 & 2 & $\begin{array}{l}1 \\
7\end{array}$ & $\begin{array}{l}1 \\
5\end{array}$ & $\begin{array}{l}1 \\
6\end{array}$ & 1 & 8 & $\begin{array}{l}1 \\
0\end{array}$ & $\begin{array}{l}1 \\
4 \\
\end{array}$ & $\begin{array}{l}1 \\
3 \\
\end{array}$ & 7 & $\begin{array}{l}1 \\
2 \\
\end{array}$ & $\begin{array}{l}2 \\
2 \\
\end{array}$ & 9 & $\begin{array}{l}2 \\
1\end{array}$ & 3 & $\begin{array}{l}1 \\
9 \\
\end{array}$ & \begin{tabular}{|l|l}
0,370 \\
339 \\
\end{tabular} \\
\hline 26-й & 3 & 1 & $\begin{array}{l}1 \\
0\end{array}$ & $\begin{array}{l}1 \\
4 \\
\end{array}$ & 4 & 5 & $\begin{array}{l}1 \\
2\end{array}$ & 7 & $\begin{array}{l}1 \\
9\end{array}$ & $\begin{array}{l}1 \\
7\end{array}$ & 6 & $\begin{array}{l}2 \\
1\end{array}$ & $\begin{array}{l}1 \\
3\end{array}$ & $\begin{array}{l}2 \\
2\end{array}$ & 8 & $\begin{array}{l}1 \\
6\end{array}$ & 9 & $\begin{array}{l}2 \\
0\end{array}$ & $\begin{array}{l}1 \\
8\end{array}$ & $\begin{array}{l}1 \\
5\end{array}$ & 2 & $\begin{array}{l}1 \\
1\end{array}$ & \begin{tabular}{|l|}
0,648 \\
305 \\
\end{tabular} \\
\hline 27-й & 7 & 2 & $\begin{array}{l}1 \\
9 \\
\end{array}$ & 8 & 1 & $\begin{array}{l}1 \\
5 \\
\end{array}$ & 6 & $\begin{array}{l}2 \\
0 \\
\end{array}$ & $\begin{array}{l}1 \\
7 \\
\end{array}$ & $\begin{array}{l}1 \\
6 \\
\end{array}$ & 3 & 9 & $\begin{array}{l}1 \\
4 \\
\end{array}$ & $\begin{array}{l}1 \\
3 \\
\end{array}$ & $\begin{array}{l}1 \\
8 \\
\end{array}$ & 5 & $\begin{array}{l}2 \\
2 \\
\end{array}$ & $\begin{array}{l}1 \\
1 \\
\end{array}$ & $\begin{array}{l}1 \\
2 \\
\end{array}$ & $\begin{array}{l}2 \\
1 \\
\end{array}$ & $\begin{array}{l}1 \\
0 \\
\end{array}$ & 4 & \begin{tabular}{|l|}
0,537 \\
288 \\
\end{tabular} \\
\hline 28-й & 8 & 3 & $\begin{array}{l}1 \\
6\end{array}$ & 9 & 1 & $\begin{array}{l}1 \\
7\end{array}$ & 6 & 7 & $\begin{array}{l}1 \\
9\end{array}$ & $\begin{array}{l}1 \\
8\end{array}$ & 2 & $\begin{array}{l}1 \\
0\end{array}$ & $\begin{array}{l}1 \\
5\end{array}$ & $\begin{array}{l}2 \\
0\end{array}$ & $\begin{array}{l}1 \\
4 \\
\end{array}$ & 4 & $\begin{array}{l}2 \\
2\end{array}$ & $\begin{array}{l}1 \\
2 \\
\end{array}$ & $\begin{array}{l}1 \\
3\end{array}$ & $\begin{array}{l}2 \\
1 \\
\end{array}$ & $\begin{array}{l}1 \\
1\end{array}$ & 5 & \begin{tabular}{|l|}
0,624 \\
576
\end{tabular} \\
\hline 29-й & 4 & $\begin{array}{l}1 \\
1 \\
\end{array}$ & 7 & $\begin{array}{l}1 \\
0 \\
\end{array}$ & 1 & 9 & 2 & $\begin{array}{l}1 \\
7 \\
\end{array}$ & $\begin{array}{l}1 \\
4 \\
\end{array}$ & $\begin{array}{l}2 \\
1 \\
\end{array}$ & 8 & $\begin{array}{l}1 \\
9 \\
\end{array}$ & 6 & $\begin{array}{l}2 \\
0 \\
\end{array}$ & \begin{tabular}{|l|}
1 \\
3 \\
\end{tabular} & $\begin{array}{l}2 \\
2 \\
\end{array}$ & 3 & $\begin{array}{l}1 \\
8 \\
\end{array}$ & $\begin{array}{l}1 \\
2 \\
\end{array}$ & $\begin{array}{l}1 \\
6 \\
\end{array}$ & 5 & $\begin{array}{l}1 \\
5 \\
\end{array}$ & \begin{tabular}{|l|}
0,561 \\
299 \\
\end{tabular} \\
\hline 30-й & 1 & 3 & $\begin{array}{l}2 \\
1\end{array}$ & $\begin{array}{l}1 \\
0 \\
\end{array}$ & 8 & 9 & 7 & $\begin{array}{l}1 \\
4 \\
\end{array}$ & $\begin{array}{l}1 \\
2\end{array}$ & $\begin{array}{l}1 \\
3 \\
\end{array}$ & $\begin{array}{l}1 \\
1 \\
\end{array}$ & $\begin{array}{l}2 \\
2 \\
\end{array}$ & $\begin{array}{l}1 \\
5\end{array}$ & $\begin{array}{l}1 \\
7\end{array}$ & 6 & $\begin{array}{l}1 \\
8 \\
\end{array}$ & $\begin{array}{l}1 \\
9\end{array}$ & $\begin{array}{l}1 \\
6 \\
\end{array}$ & 5 & $\begin{array}{l}2 \\
0\end{array}$ & 2 & 4 & \begin{tabular}{|l|}
0,652 \\
825 \\
\end{tabular} \\
\hline 31-й & $\begin{array}{l}1 \\
3 \\
\end{array}$ & 4 & $\begin{array}{l}1 \\
4\end{array}$ & $\begin{array}{l}1 \\
6\end{array}$ & 3 & $\begin{array}{l}2 \\
2\end{array}$ & 7 & $\begin{array}{l}2 \\
1\end{array}$ & 8 & $\begin{array}{l}7 \\
7\end{array}$ & 5 & $\begin{array}{l}1 \\
5\end{array}$ & 6 & $\begin{array}{l}1 \\
2\end{array}$ & $\begin{array}{l}1 \\
1 \\
\end{array}$ & $\begin{array}{l}1 \\
8 \\
\end{array}$ & \begin{tabular}{l|}
1 \\
0
\end{tabular} & 9 & $\begin{array}{l}2 \\
0\end{array}$ & 1 & 2 & $\begin{array}{l}1 \\
9 \\
9\end{array}$ & \begin{tabular}{|l}
0,361 \\
582 \\
\end{tabular} \\
\hline 32-й & 9 & 2 & $\begin{array}{l}1 \\
0 \\
\end{array}$ & $\begin{array}{l}1 \\
4 \\
\end{array}$ & 1 & $\begin{array}{l}1 \\
6 \\
\end{array}$ & $\begin{array}{l}1 \\
5 \\
\end{array}$ & $\begin{array}{l}1 \\
9 \\
\end{array}$ & $\begin{array}{l}1 \\
7 \\
\end{array}$ & $\begin{array}{l}2 \\
0 \\
\end{array}$ & 3 & 4 & $\begin{array}{l}1 \\
1 \\
\end{array}$ & $\begin{array}{l}1 \\
3 \\
\end{array}$ & $\begin{array}{l}1 \\
2 \\
\end{array}$ & $\begin{array}{l}1 \\
8 \\
\end{array}$ & 5 & $\begin{array}{l}2 \\
1 \\
\end{array}$ & 7 & $\begin{array}{l}2 \\
2 \\
\end{array}$ & 6 & 8 & \begin{tabular}{|l|}
0,519 \\
774 \\
\end{tabular} \\
\hline 33-й & 1 & 9 & $\begin{array}{l}1 \\
0\end{array}$ & $\begin{array}{l}1 \\
2\end{array}$ & $\begin{array}{l}1 \\
1\end{array}$ & 7 & 6 & 5 & $\begin{array}{l}1 \\
5\end{array}$ & $\begin{array}{l}1 \\
4\end{array}$ & $\begin{array}{l}1 \\
3 \\
\end{array}$ & $\begin{array}{l}1 \\
7\end{array}$ & $\begin{array}{l}1 \\
6\end{array}$ & $\begin{array}{l}1 \\
8\end{array}$ & $\begin{array}{l}1 \\
9 \\
\end{array}$ & 8 & $\begin{array}{l}2 \\
1\end{array}$ & 4 & $\begin{array}{l}2 \\
2\end{array}$ & $\begin{array}{l}2 \\
0 \\
\end{array}$ & 3 & 2 & \begin{tabular}{|l}
0,615 \\
537
\end{tabular} \\
\hline 34-й & $\begin{array}{l}1 \\
2\end{array}$ & 2 & $\begin{array}{l}1 \\
3\end{array}$ & $\begin{array}{l}1 \\
1\end{array}$ & $\begin{array}{l}1 \\
0\end{array}$ & 1 & $\begin{array}{l}1 \\
8\end{array}$ & 8 & $\begin{array}{l}1 \\
9\end{array}$ & $\begin{array}{l}1 \\
7\end{array}$ & 9 & 7 & $\begin{array}{l}1 \\
4\end{array}$ & $\begin{array}{l}2 \\
0\end{array}$ & 6 & 3 & $\begin{array}{l}2 \\
1\end{array}$ & $\begin{array}{l}1 \\
6\end{array}$ & $\begin{array}{l}2 \\
2\end{array}$ & $\begin{array}{l}1 \\
5\end{array}$ & 4 & 5 & \begin{tabular}{|l|}
0,652 \\
26 \\
\end{tabular} \\
\hline 35-й & 4 & 3 & $\begin{array}{l}1 \\
5 \\
\end{array}$ & 5 & 6 & 7 & $\begin{array}{l}1 \\
4 \\
\end{array}$ & $\begin{array}{l}1 \\
6 \\
\end{array}$ & 8 & $\begin{array}{l}1 \\
1 \\
\end{array}$ & 1 & $\begin{array}{l}2 \\
0 \\
\end{array}$ & $\begin{array}{l}1 \\
7 \\
\end{array}$ & $\begin{array}{l}2 \\
1 \\
\end{array}$ & $\begin{array}{l}1 \\
2 \\
\end{array}$ & 9 & \begin{tabular}{l|}
1 \\
0 \\
\end{tabular} & 2 & $\begin{array}{l}2 \\
2 \\
\end{array}$ & $\begin{array}{l}1 \\
3 \\
\end{array}$ & $\begin{array}{l}1 \\
8 \\
\end{array}$ & $\begin{array}{l}1 \\
9 \\
\end{array}$ & \begin{tabular}{|l|}
0,509 \\
605 \\
\end{tabular} \\
\hline 36-й & 2 & 4 & $\begin{array}{l}1 \\
1\end{array}$ & $\begin{array}{l}1 \\
2 \\
\end{array}$ & 1 & $\begin{array}{l}1 \\
4\end{array}$ & $\begin{array}{l}1 \\
9 \\
\end{array}$ & $\begin{array}{l}2 \\
0\end{array}$ & $\begin{array}{l}2 \\
1\end{array}$ & 5 & $\begin{array}{l}1 \\
8 \\
\end{array}$ & $\begin{array}{l}1 \\
7\end{array}$ & 6 & $\begin{array}{l}2 \\
2\end{array}$ & 7 & 8 & $\begin{array}{l}1 \\
0\end{array}$ & 3 & 9 & $\begin{array}{l}1 \\
3 \\
\end{array}$ & $\begin{array}{l}1 \\
5\end{array}$ & $\begin{array}{l}1 \\
6\end{array}$ & \begin{tabular}{|l}
0,478 \\
814
\end{tabular} \\
\hline 37-й & $\begin{array}{l}1 \\
0\end{array}$ & 9 & $\begin{array}{l}1 \\
7\end{array}$ & $\begin{array}{l}1 \\
1\end{array}$ & 4 & 5 & \begin{tabular}{|l|}
1 \\
5
\end{tabular} & $\begin{array}{l}1 \\
4\end{array}$ & $\begin{array}{l}1 \\
6\end{array}$ & $\begin{array}{l}1 \\
3\end{array}$ & 1 & 2 & $\begin{array}{l}1 \\
9\end{array}$ & $\begin{array}{l}2 \\
2\end{array}$ & 3 & $\begin{array}{l}1 \\
8\end{array}$ & 6 & 7 & $\overline{8}$ & $\begin{array}{l}1 \\
2\end{array}$ & $\begin{array}{l}2 \\
0\end{array}$ & $\begin{array}{l}2 \\
1\end{array}$ & \begin{tabular}{|l}
0,496 \\
61
\end{tabular} \\
\hline 38-й & 1 & 6 & 7 & 5 & 4 & $\begin{array}{l}1 \\
3 \\
\end{array}$ & $\begin{array}{l}1 \\
0\end{array}$ & 9 & $\begin{array}{l}1 \\
2\end{array}$ & $\begin{array}{l}1 \\
1\end{array}$ & 4 & 8 & 2 & $\begin{array}{l}1 \\
4 \\
\end{array}$ & $\begin{array}{l}1 \\
6 \\
\end{array}$ & 4 & $\begin{array}{l}1 \\
5\end{array}$ & $\begin{array}{l}1 \\
8 \\
\end{array}$ & $\begin{array}{l}1 \\
7\end{array}$ & $\begin{array}{l}1 \\
9\end{array}$ & 3 & $\begin{array}{l}2 \\
0 \\
\end{array}$ & \begin{tabular}{|l}
0,490 \\
667 \\
\end{tabular} \\
\hline 39-й & 2 & 5 & $\begin{array}{l}1 \\
6\end{array}$ & $\begin{array}{l}1 \\
0\end{array}$ & 9 & $\begin{array}{l}1 \\
5\end{array}$ & $\begin{array}{l}1 \\
9\end{array}$ & $\begin{array}{l}1 \\
1\end{array}$ & 8 & 7 & 1 & $\begin{array}{l}1 \\
8\end{array}$ & 6 & $\begin{array}{l}2 \\
1\end{array}$ & $\begin{array}{l}1 \\
4 \\
\end{array}$ & $\begin{array}{l}2 \\
2\end{array}$ & $\begin{array}{l}1 \\
2\end{array}$ & $\begin{array}{l}1 \\
7\end{array}$ & 4 & $\begin{array}{l}2 \\
0\end{array}$ & 3 & $\begin{array}{l}1 \\
3 \\
\end{array}$ & \begin{tabular}{|l}
0,486 \\
723
\end{tabular} \\
\hline 40-й & 1 & 2 & $\begin{array}{l}1 \\
5\end{array}$ & \begin{tabular}{|l|}
1 \\
2 \\
\end{tabular} & $\begin{array}{l}1 \\
3\end{array}$ & $\begin{array}{l}1 \\
4\end{array}$ & \begin{tabular}{|l|}
6 \\
\end{tabular} & $\begin{array}{l}1 \\
6\end{array}$ & 3 & 3 & 4 & 7 & 5 & 4 & 8 & 9 & $\begin{array}{l}1 \\
0 \\
\end{array}$ & $\begin{array}{l}1 \\
1\end{array}$ & $\begin{array}{l}1 \\
8\end{array}$ & $\begin{array}{l}1 \\
7\end{array}$ & $\begin{array}{l}2 \\
0\end{array}$ & $\begin{array}{l}1 \\
9\end{array}$ & \begin{tabular}{|l}
0,477 \\
671
\end{tabular} \\
\hline 41-й & 1 & 3 & $\begin{array}{l}2 \\
2\end{array}$ & 4 & 2 & 5 & 6 & $\begin{array}{l}1 \\
3\end{array}$ & $\begin{array}{l}1 \\
5\end{array}$ & $\begin{array}{l}1 \\
6\end{array}$ & $\begin{array}{l}1 \\
7\end{array}$ & $\begin{array}{l}1 \\
8\end{array}$ & 7 & $\begin{array}{l}1 \\
9 \\
\end{array}$ & $\begin{array}{l}2 \\
0 \\
\end{array}$ & 8 & 9 & $\begin{array}{l}1 \\
0\end{array}$ & $\begin{array}{l}1 \\
1\end{array}$ & $\begin{array}{l}1 \\
2\end{array}$ & $\begin{array}{l}2 \\
1\end{array}$ & $\begin{array}{l}1 \\
4\end{array}$ & \begin{tabular}{|l}
0,592 \\
09
\end{tabular} \\
\hline 42-й & 1 & $\begin{array}{l}1 \\
8\end{array}$ & $\begin{array}{l}1 \\
0\end{array}$ & \begin{tabular}{|l|}
1 \\
7
\end{tabular} & 9 & $\begin{array}{l}1 \\
3\end{array}$ & \begin{tabular}{|l|}
1 \\
6
\end{tabular} & $\begin{array}{l}1 \\
9\end{array}$ & 6 & 7 & $\begin{array}{l}1 \\
5\end{array}$ & 2 & $\begin{array}{l}1 \\
4\end{array}$ & 5 & 4 & $\begin{array}{l}2 \\
0\end{array}$ & $\begin{array}{l}1 \\
1\end{array}$ & 8 & $\begin{array}{l}2 \\
1\end{array}$ & $\begin{array}{l}1 \\
2 \\
\end{array}$ & $\begin{array}{l}2 \\
2\end{array}$ & 3 & \begin{tabular}{|l|l|}
0,652 \\
26
\end{tabular} \\
\hline 43-й & $\begin{array}{l}1 \\
0 \\
\end{array}$ & 8 & 3 & \begin{tabular}{|l|}
6 \\
\end{tabular} & 7 & 9 & $\begin{array}{l}1 \\
0 \\
\end{array}$ & $\begin{array}{l}1 \\
0 \\
\end{array}$ & 1 & 4 & 1 & 3 & 1 & 5 & 3 & 3 & 2 & 1 & $\frac{1}{2}$ & 8 & 5 & 5 & \begin{tabular}{|l|}
0,228 \\
348
\end{tabular} \\
\hline 44-й & $\begin{array}{l}1 \\
0\end{array}$ & 2 & 4 & \begin{tabular}{|l|}
1 \\
0
\end{tabular} & 6 & 7 & 8 & 2 & 1 & 9 & 1 & 1 & 1 & 4 & 1 & 1 & 5 & 1 & 3 & 5 & 5 & 4 & \begin{tabular}{|l}
0,365 \\
285 \\
\end{tabular} \\
\hline 45-й & $\begin{array}{l}1 \\
1\end{array}$ & 4 & $\begin{array}{l}1 \\
8\end{array}$ & \begin{tabular}{|l|}
5 \\
\end{tabular} & 1 & 2 & 3 & $\begin{array}{l}1 \\
6\end{array}$ & $\begin{array}{l}1 \\
7\end{array}$ & $\begin{array}{l}2 \\
0\end{array}$ & 6 & $\begin{array}{l}1 \\
9\end{array}$ & $\begin{array}{l}1 \\
0\end{array}$ & 9 & $\begin{array}{l}1 \\
5 \\
\end{array}$ & $\begin{array}{l}1 \\
4\end{array}$ & $\begin{array}{l}2 \\
1 \\
\end{array}$ & $\begin{array}{l}1 \\
2\end{array}$ & $\begin{array}{l}1 \\
3\end{array}$ & $\begin{array}{l}2 \\
2\end{array}$ & 7 & 8 & \begin{tabular}{|l|}
0,605 \\
367
\end{tabular} \\
\hline 46-й & 4 & 2 & $\begin{array}{l}2 \\
1\end{array}$ & 7 & $\begin{array}{l}1 \\
8\end{array}$ & $\begin{array}{l}1 \\
7\end{array}$ & $\begin{array}{l}1 \\
2 \\
\end{array}$ & 6 & $\begin{array}{l}1 \\
1\end{array}$ & $\begin{array}{l}1 \\
0\end{array}$ & 5 & 1 & $\begin{array}{l}1 \\
9\end{array}$ & 9 & 8 & $\begin{array}{l}1 \\
5 \\
\end{array}$ & $\begin{array}{l}2 \\
2 \\
\end{array}$ & $\begin{array}{l}1 \\
4 \\
\end{array}$ & $\begin{array}{l}1 \\
6\end{array}$ & $\begin{array}{l}2 \\
0\end{array}$ & $\begin{array}{l}1 \\
3 \\
\end{array}$ & 3 & \begin{tabular}{|l}
0,691 \\
243 \\
\end{tabular} \\
\hline 47-й & 3 & $\begin{array}{l}1 \\
1\end{array}$ & $\begin{array}{l}1 \\
6\end{array}$ & 8 & $\begin{array}{l}1 \\
2\end{array}$ & 1 & 2 & 4 & 6 & $\begin{array}{l}1 \\
9\end{array}$ & 9 & 5 & $\begin{array}{l}1 \\
3\end{array}$ & 9 & 7 & $\begin{array}{l}1 \\
9\end{array}$ & 6 & $\begin{array}{l}1 \\
4\end{array}$ & $\begin{array}{l}1 \\
8\end{array}$ & \begin{tabular}{|l|}
1 \\
7
\end{tabular} & $\begin{array}{l}1 \\
5\end{array}$ & $\begin{array}{l}1 \\
0\end{array}$ & \begin{tabular}{|l|}
0,714 \\
306
\end{tabular} \\
\hline 48-й & 7 & 4 & $\begin{array}{l}1 \\
5 \\
\end{array}$ & 5 & 3 & $\begin{array}{l}1 \\
6\end{array}$ & \begin{tabular}{|l|}
8 \\
\end{tabular} & 8 & 6 & $\begin{array}{l}1 \\
0\end{array}$ & 9 & $\begin{array}{l}1 \\
2\end{array}$ & 2 & $\begin{array}{l}1 \\
1\end{array}$ & 3 & $\begin{array}{l}2 \\
0\end{array}$ & $\begin{array}{l}1 \\
9\end{array}$ & $\begin{array}{l}1 \\
3\end{array}$ & $\begin{array}{l}1 \\
4\end{array}$ & $\begin{array}{l}1 \\
8\end{array}$ & $\begin{array}{l}1 \\
7\end{array}$ & 1 & \begin{tabular}{|l|}
0,816 \\
846
\end{tabular} \\
\hline 49-й & 6 & 5 & $\begin{array}{l}1 \\
5\end{array}$ & 6 & $\begin{array}{l}1 \\
8\end{array}$ & 7 & $\begin{array}{l}1 \\
9\end{array}$ & 3 & 8 & $\begin{array}{l}1 \\
9\end{array}$ & 9 & $\begin{array}{l}1 \\
4\end{array}$ & 2 & $\begin{array}{l}1 \\
3 \\
\end{array}$ & $\begin{array}{l}1 \\
6 \\
\end{array}$ & $\begin{array}{l}1 \\
8 \\
\end{array}$ & 4 & $\begin{array}{l}1 \\
0\end{array}$ & $\begin{array}{l}1 \\
2 \\
\end{array}$ & $\begin{array}{l}1 \\
7 \\
\end{array}$ & $\begin{array}{l}1 \\
1\end{array}$ & 1 & \begin{tabular}{|l}
0,605 \\
739
\end{tabular} \\
\hline 50-й & $\begin{array}{l}1 \\
7\end{array}$ & $\begin{array}{l}1 \\
4\end{array}$ & $\begin{array}{l}2 \\
1\end{array}$ & 1 & $\begin{array}{l}2 \\
2\end{array}$ & 8 & 9 & $\begin{array}{l}2 \\
0\end{array}$ & 5 & 7 & 6 & $\begin{array}{l}1 \\
0\end{array}$ & $\begin{array}{l}1 \\
2\end{array}$ & $\begin{array}{l}1 \\
3\end{array}$ & $\begin{array}{l}1 \\
1 \\
\end{array}$ & $\begin{array}{l}1 \\
5\end{array}$ & 2 & $\begin{array}{l}1 \\
6\end{array}$ & $\begin{array}{l}1 \\
8 \\
\end{array}$ & $\begin{array}{l}1 \\
9\end{array}$ & 3 & 4 & \begin{tabular}{|l}
0,436 \\
441
\end{tabular} \\
\hline 51-й & $\begin{array}{l}1 \\
3 \\
\end{array}$ & 1 & $\begin{array}{l}2 \\
2 \\
\end{array}$ & \begin{tabular}{|l|}
1 \\
5 \\
\end{tabular} & 9 & 8 & $\begin{array}{l}2 \\
1 \\
\end{array}$ & 6 & $\begin{array}{l}1 \\
0 \\
\end{array}$ & 7 & $\begin{array}{l}1 \\
2 \\
\end{array}$ & $\begin{array}{l}1 \\
1 \\
\end{array}$ & $\begin{array}{l}1 \\
6 \\
\end{array}$ & $\begin{array}{l}1 \\
4 \\
\end{array}$ & $\begin{array}{l}1 \\
7 \\
\end{array}$ & 2 & $\begin{array}{l}2 \\
0 \\
\end{array}$ & $\begin{array}{l}1 \\
8 \\
\end{array}$ & $\begin{array}{l}1 \\
9 \\
\end{array}$ & 5 & 4 & 3 & \begin{tabular}{|l}
0,531 \\
921 \\
\end{tabular} \\
\hline 52-й & 3 & 1 & $\begin{array}{l}2 \\
2\end{array}$ & \begin{tabular}{|l|}
1 \\
2
\end{tabular} & 4 & 9 & 8 & $\begin{array}{l}1 \\
0\end{array}$ & 5 & $\begin{array}{l}1 \\
5\end{array}$ & 6 & $\begin{array}{l}1 \\
3\end{array}$ & $\begin{array}{l}1 \\
6\end{array}$ & $\begin{array}{l}1 \\
4\end{array}$ & $\begin{array}{l}1 \\
1\end{array}$ & $\begin{array}{l}1 \\
7\end{array}$ & $\begin{array}{l}2 \\
0\end{array}$ & 7 & $\begin{array}{l}1 \\
8\end{array}$ & $\begin{array}{l}1 \\
9\end{array}$ & $\begin{array}{l}2 \\
1\end{array}$ & 2 & \begin{tabular}{|l|}
0,727 \\
966
\end{tabular} \\
\hline 53-й & $\begin{array}{l}1 \\
4\end{array}$ & $\begin{array}{l}1 \\
7\end{array}$ & $\begin{array}{l}1 \\
8\end{array}$ & \begin{tabular}{|l}
1 \\
2
\end{tabular} & 5 & 6 & 2 & $\begin{array}{l}1 \\
9\end{array}$ & 7 & $\begin{array}{l}1 \\
6\end{array}$ & 1 & $\begin{array}{l}1 \\
1\end{array}$ & $\begin{array}{l}1 \\
5\end{array}$ & $\begin{array}{l}1 \\
0\end{array}$ & $\begin{array}{l}2 \\
0 \\
0\end{array}$ & 4 & $\begin{array}{l}1 \\
9 \\
\end{array}$ & 3 & 8 & $\begin{array}{l}1 \\
3\end{array}$ & 9 & 1 & \begin{tabular}{|l|l}
0,386 \\
376
\end{tabular} \\
\hline 54-й & 8 & 1 & $\begin{array}{l}2 \\
1\end{array}$ & 2 & $\begin{array}{l}1 \\
0\end{array}$ & 4 & $\begin{array}{l}1 \\
3 \\
\end{array}$ & $\begin{array}{l}1 \\
2\end{array}$ & 5 & $\begin{array}{l}2 \\
0\end{array}$ & $\begin{array}{l}1 \\
9 \\
\end{array}$ & 6 & $\begin{array}{l}1 \\
8\end{array}$ & 7 & $\begin{array}{l}2 \\
2 \\
\end{array}$ & 9 & $\begin{array}{l}1 \\
7 \\
\end{array}$ & $\begin{array}{l}1 \\
6\end{array}$ & $\begin{array}{l}1 \\
5 \\
\end{array}$ & $\begin{array}{l}1 \\
4\end{array}$ & 3 & $\begin{array}{l}1 \\
1\end{array}$ & \begin{tabular}{|l}
0,578 \\
531
\end{tabular} \\
\hline 55-й & 7 & 8 & $\begin{array}{l}1 \\
3\end{array}$ & \begin{tabular}{|l|}
1 \\
4
\end{tabular} & 9 & 8 & $\begin{array}{l}1 \\
1\end{array}$ & $\begin{array}{l}1 \\
9\end{array}$ & 0 & 1 & 1 & $\begin{array}{l}1 \\
2\end{array}$ & $\begin{array}{l}1 \\
5\end{array}$ & 2 & $\begin{array}{l}1 \\
6\end{array}$ & $\begin{array}{l}1 \\
7\end{array}$ & 2 & 5 & 4 & 3 & 5 & 6 & \begin{tabular}{|l}
0,252 \\
898
\end{tabular} \\
\hline 1-й & 5 & 8 & 6 & \begin{tabular}{|l|}
2 \\
\end{tabular} & 7 & 9 & $\frac{1}{1}$ & 4 & 1 & 1 & $\begin{array}{l}1 \\
7\end{array}$ & 1 & 1 & 1 & 3 & 1 & 1 & $\begin{array}{l}2 \\
0\end{array}$ & \begin{tabular}{|l}
1 \\
6 \\
\end{tabular} & $\begin{array}{l}1 \\
2 \\
\end{array}$ & $\begin{array}{l}2 \\
0 \\
\end{array}$ & 1 & 0,82 \\
\hline
\end{tabular}




\begin{tabular}{|c|c|c|c|c|c|c|}
\hline \multirow{4}{*}{ Impact Factor: } & ISRA (India) & $=3.117$ & SIS (USA) & $=0.912$ & ICV (Poland) & $=6.630$ \\
\hline & ISI (Dubai, UAE & $=0.829$ & РИНЦ (Russia) & $=0.156$ & PIF (India) & $=1.940$ \\
\hline & GIF (Australia) & $=0.564$ & ESJI (KZ) & $=8.716$ & IBI (India) & $=4.260$ \\
\hline & JIF & $=1.500$ & SJIF (Morocco) & $=5.667$ & OAJI (USA) & $=0.350$ \\
\hline
\end{tabular}

Table 6 - Results of evaluation of competence of buyers surveyed by competitivenesses assessment and demand for children's shoes

\begin{tabular}{|c|c|c|c|c|c|c|c|c|c|c|c|c|c|c|c|c|c|c|c|c|c|c|c|}
\hline \multirow{2}{*}{\begin{tabular}{l} 
АНАЛ \\
\multicolumn{1}{|c|}{} \\
$\begin{array}{l}\text { Экспер } \\
\text { ты }\end{array}$ \\
\end{tabular}} & \multirow[b]{2}{*}{$\begin{array}{l}\text { Фа } \\
\text { кто } \\
\text { ры }\end{array}$} & \multicolumn{3}{|c|}{ Исключить } & \multicolumn{2}{|c|}{ CTAPT } & & & & & & & & & & & & & & & & & \\
\hline & & 1 & 2 & 3 & 4 & 5 & 6 & \multicolumn{2}{|c|}{7} & 8 & 9 & $\begin{array}{l}1 \\
\mathbf{0}\end{array}$ & $\begin{array}{l}1 \\
1\end{array}$ & $\begin{array}{l}1 \\
2\end{array}$ & $\begin{array}{l}1 \\
3\end{array}$ & $\begin{array}{l}1 \\
4\end{array}$ & $\begin{array}{l}1 \\
5\end{array}$ & $\begin{array}{l}1 \\
6\end{array}$ & $\begin{array}{l}1 \\
7\end{array}$ & $\begin{array}{l}1 \\
8\end{array}$ & $\begin{array}{l}1 \\
9\end{array}$ & $\begin{array}{l}2 \\
0\end{array}$ & $\mathrm{~W}_{\mathrm{i}}$ \\
\hline 1 & & 13 & $\begin{array}{l}1 \\
1 \\
\end{array}$ & 9 & 1 & 2 & 6 & & & 5 & 7 & 4 & $\begin{array}{l}1 \\
2 \\
\end{array}$ & $\begin{array}{l}1 \\
4\end{array}$ & 8 & $\begin{array}{l}1 \\
7\end{array}$ & $\begin{array}{l}1 \\
5\end{array}$ & $\begin{array}{l}1 \\
8 \\
\end{array}$ & $\begin{array}{l}1 \\
6 \\
\end{array}$ & $\begin{array}{l}1 \\
0\end{array}$ & & & $\begin{array}{l}0,620 \\
227\end{array}$ \\
\hline 2 & & 4 & $\begin{array}{l}1 \\
8 \\
\end{array}$ & 5 & $\begin{array}{l}1 \\
7 \\
\end{array}$ & 1 & $\begin{array}{l}1 \\
6 \\
\end{array}$ & & & $\begin{array}{l}1 \\
3 \\
\end{array}$ & 2 & $\begin{array}{l}1 \\
2 \\
\end{array}$ & $\begin{array}{l}1 \\
1 \\
\end{array}$ & $\begin{array}{l}1 \\
5\end{array}$ & $\begin{array}{l}1 \\
4 \\
\end{array}$ & 8 & 7 & 9 & $\begin{array}{l}1 \\
0\end{array}$ & 6 & & & $\begin{array}{l}0,680 \\
083\end{array}$ \\
\hline 3 & & 3 & 2 & 4 & 9 & $\begin{array}{l}1 \\
0\end{array}$ & $\overline{1}$ & & & 6 & 8 & $\begin{array}{l}1 \\
1 \\
\end{array}$ & $\begin{array}{l}1 \\
7 \\
\end{array}$ & $\begin{array}{l}1 \\
2\end{array}$ & 7 & $\begin{array}{l}1 \\
3 \\
\end{array}$ & $\begin{array}{l}1 \\
6 \\
\end{array}$ & $\begin{array}{l}1 \\
5 \\
\end{array}$ & $\begin{array}{l}1 \\
4 \\
\end{array}$ & $\begin{array}{l}1 \\
8 \\
\end{array}$ & & & $\begin{array}{l}0,724 \\
458 \\
\end{array}$ \\
\hline 4 & & 8 & $\begin{array}{l}1 \\
3 \\
\end{array}$ & 4 & 3 & 9 & 1 & & & $\begin{array}{l}1 \\
2\end{array}$ & 2 & 5 & $\begin{array}{l}1 \\
4 \\
\end{array}$ & 6 & 7 & $\begin{array}{l}1 \\
5 \\
\end{array}$ & $\begin{array}{l}1 \\
6 \\
\end{array}$ & $\begin{array}{l}1 \\
7\end{array}$ & $\begin{array}{l}1 \\
8 \\
\end{array}$ & $\begin{array}{l}1 \\
1 \\
\end{array}$ & & & $\begin{array}{l}0,640 \\
867\end{array}$ \\
\hline 5 & & 4 & 3 & 5 & 7 & 2 & 1 & & & $\begin{array}{l}1 \\
2\end{array}$ & 8 & 9 & $\begin{array}{l}1 \\
0\end{array}$ & $\begin{array}{l}1 \\
3\end{array}$ & $\begin{array}{l}1 \\
5\end{array}$ & $\begin{array}{l}1 \\
4\end{array}$ & $\begin{array}{l}1 \\
8\end{array}$ & $\begin{array}{l}1 \\
7\end{array}$ & $\begin{array}{l}1 \\
6\end{array}$ & $\begin{array}{l}1 \\
1\end{array}$ & & & $\begin{array}{l}0,723 \\
426\end{array}$ \\
\hline 6 & & 5 & $\begin{array}{l}1 \\
3 \\
\end{array}$ & 1 & 3 & 4 & 2 & & & $\begin{array}{l}1 \\
2 \\
\end{array}$ & 6 & 7 & $\begin{array}{l}1 \\
8 \\
\end{array}$ & 9 & 8 & $\begin{array}{l}1 \\
4 \\
\end{array}$ & $\begin{array}{l}1 \\
5\end{array}$ & $\begin{array}{l}1 \\
6 \\
\end{array}$ & $\begin{array}{l}1 \\
7\end{array}$ & $\begin{array}{l}1 \\
0 \\
\end{array}$ & & & $\begin{array}{l}0,652 \\
219 \\
\end{array}$ \\
\hline 7 & & 1 & 9 & 4 & 3 & 8 & $\begin{array}{l}1 \\
0\end{array}$ & & & 6 & 2 & $\begin{array}{l}1 \\
1 \\
\end{array}$ & $\begin{array}{l}1 \\
3 \\
\end{array}$ & $\begin{array}{l}1 \\
2\end{array}$ & $\begin{array}{l}1 \\
5 \\
\end{array}$ & $\begin{array}{l}1 \\
4 \\
\end{array}$ & $\begin{array}{l}1 \\
6 \\
\end{array}$ & $\begin{array}{l}1 \\
8 \\
\end{array}$ & $\begin{array}{l}1 \\
7\end{array}$ & 7 & & & $\begin{array}{l}0,734 \\
778 \\
\end{array}$ \\
\hline 8 & & 11 & $\begin{array}{l}1 \\
2 \\
\end{array}$ & 3 & 2 & 9 & $\begin{array}{l}1 \\
0 \\
\end{array}$ & & & 5 & 1 & $\begin{array}{l}1 \\
3 \\
\end{array}$ & $\begin{array}{l}1 \\
5 \\
\end{array}$ & 6 & 7 & $\begin{array}{l}1 \\
4 \\
\end{array}$ & $\begin{array}{l}1 \\
6 \\
\end{array}$ & $\begin{array}{l}1 \\
7 \\
\end{array}$ & $\begin{array}{l}1 \\
8 \\
\end{array}$ & 8 & & & $\begin{array}{l}0,631 \\
579 \\
\end{array}$ \\
\hline 9 & & 18 & $\begin{array}{l}1 \\
7 \\
\end{array}$ & 1 & 2 & 5 & 3 & & & 6 & 7 & $\begin{array}{l}1 \\
0\end{array}$ & $\begin{array}{l}1 \\
1\end{array}$ & 8 & $\begin{array}{l}1 \\
2 \\
\end{array}$ & $\begin{array}{l}1 \\
6\end{array}$ & $\begin{array}{l}1 \\
4 \\
\end{array}$ & $\begin{array}{l}1 \\
3 \\
\end{array}$ & $\begin{array}{l}1 \\
5\end{array}$ & 9 & & & $\begin{array}{l}0,622 \\
291\end{array}$ \\
\hline 10 & & 4 & 3 & \begin{tabular}{|l}
1 \\
6 \\
\end{tabular} & 5 & 7 & 1 & & & 8 & 9 & $\begin{array}{l}1 \\
0\end{array}$ & $\begin{array}{l}1 \\
1\end{array}$ & $\begin{array}{l}1 \\
2 \\
\end{array}$ & $\begin{array}{l}1 \\
4 \\
\end{array}$ & $\begin{array}{l}1 \\
3 \\
\end{array}$ & $\begin{array}{l}1 \\
8 \\
\end{array}$ & $\begin{array}{l}1 \\
7\end{array}$ & 6 & $\begin{array}{l}1 \\
5\end{array}$ & & & $\begin{array}{l}0,669 \\
763\end{array}$ \\
\hline 11 & & 11 & 4 & 3 & \begin{tabular}{|l|}
1 \\
0 \\
\end{tabular} & $\begin{array}{l}1 \\
2\end{array}$ & $\begin{array}{l}1 \\
3 \\
\end{array}$ & & & 1 & 9 & 8 & $\begin{array}{l}1 \\
5 \\
\end{array}$ & $\begin{array}{l}1 \\
4\end{array}$ & $\begin{array}{l}1 \\
7\end{array}$ & $\begin{array}{l}1 \\
6 \\
\end{array}$ & 6 & 5 & 7 & $\begin{array}{l}1 \\
8 \\
\end{array}$ & & & $\begin{array}{l}0,676 \\
987 \\
\end{array}$ \\
\hline 12 & & 5 & $\begin{array}{l}1 \\
1 \\
\end{array}$ & 1 & 4 & 9 & $\begin{array}{l}1 \\
0\end{array}$ & & & 7 & 2 & $\begin{array}{l}1 \\
2 \\
\end{array}$ & $\begin{array}{l}1 \\
3 \\
\end{array}$ & 6 & $\begin{array}{l}1 \\
4 \\
\end{array}$ & 8 & $\begin{array}{l}1 \\
6 \\
\end{array}$ & $\begin{array}{l}1 \\
7 \\
\end{array}$ & $\begin{array}{l}1 \\
8 \\
\end{array}$ & $\begin{array}{l}1 \\
5 \\
\end{array}$ & & & $\begin{array}{l}0,780 \\
186 \\
\end{array}$ \\
\hline 13 & & 2 & 4 & 1 & 5 & 7 & 3 & & & $\begin{array}{l}1 \\
1 \\
\end{array}$ & 6 & $\begin{array}{l}1 \\
2 \\
\end{array}$ & 9 & $\begin{array}{l}1 \\
0\end{array}$ & $\begin{array}{l}1 \\
3 \\
\end{array}$ & $\begin{array}{l}1 \\
5 \\
\end{array}$ & $\begin{array}{l}1 \\
4 \\
\end{array}$ & $\begin{array}{l}1 \\
7\end{array}$ & $\begin{array}{l}1 \\
6 \\
\end{array}$ & $\begin{array}{l}1 \\
8 \\
\end{array}$ & & & $\begin{array}{l}0,763 \\
674 \\
\end{array}$ \\
\hline 14 & & 6 & 9 & 8 & 2 & 3 & 5 & & & $\begin{array}{l}1 \\
1 \\
\end{array}$ & 4 & $\begin{array}{l}1 \\
0 \\
\end{array}$ & $\begin{array}{l}1 \\
3 \\
\end{array}$ & 1 & $\begin{array}{l}1 \\
2 \\
\end{array}$ & $\begin{array}{l}1 \\
4 \\
\end{array}$ & $\begin{array}{l}1 \\
6 \\
\end{array}$ & $\begin{array}{l}1 \\
7\end{array}$ & $\begin{array}{l}1 \\
5 \\
\end{array}$ & $\begin{array}{l}1 \\
8 \\
\end{array}$ & & & $\begin{array}{l}0,658 \\
411 \\
\end{array}$ \\
\hline 15 & & 3 & 2 & 4 & 5 & 7 & $\overline{9}$ & & & $\begin{array}{l}1 \\
0\end{array}$ & $\begin{array}{l}1 \\
2 \\
\end{array}$ & 6 & $\begin{array}{l}1 \\
3 \\
\end{array}$ & 1 & $\begin{array}{l}1 \\
4 \\
\end{array}$ & 8 & $\begin{array}{l}1 \\
5\end{array}$ & $\begin{array}{l}1 \\
6 \\
\end{array}$ & $\begin{array}{l}1 \\
8 \\
\end{array}$ & $\begin{array}{l}1 \\
7\end{array}$ & & & $\begin{array}{l}0,598 \\
555 \\
\end{array}$ \\
\hline 16 & & 4 & $\begin{array}{l}1 \\
1 \\
\end{array}$ & 3 & \begin{tabular}{|l|}
1 \\
0 \\
\end{tabular} & $\begin{array}{l}1 \\
6 \\
\end{array}$ & 1 & & & $\begin{array}{l}1 \\
5 \\
\end{array}$ & 2 & $\begin{array}{l}1 \\
7 \\
\end{array}$ & 5 & $\begin{array}{l}1 \\
4 \\
\end{array}$ & $\begin{array}{l}1 \\
8 \\
\end{array}$ & 7 & 6 & $\begin{array}{l}1 \\
2 \\
\end{array}$ & $\begin{array}{l}1 \\
3 \\
\end{array}$ & 8 & & & $\begin{array}{l}0,840 \\
041 \\
\end{array}$ \\
\hline 17 & & 5 & $\begin{array}{l}1 \\
3 \\
\end{array}$ & 1 & 6 & $\begin{array}{l}1 \\
1\end{array}$ & 2 & & & $\begin{array}{l}1 \\
2\end{array}$ & 4 & $\begin{array}{l}1 \\
8 \\
\end{array}$ & 9 & $\begin{array}{l}1 \\
0\end{array}$ & $\begin{array}{l}1 \\
6 \\
\end{array}$ & $\begin{array}{l}1 \\
5 \\
\end{array}$ & 7 & $\begin{array}{l}1 \\
4 \\
\end{array}$ & $\begin{array}{l}1 \\
7\end{array}$ & 8 & & & $\begin{array}{l}0,822 \\
497\end{array}$ \\
\hline 18 & & 6 & 7 & 8 & \begin{tabular}{|l|}
1 \\
1 \\
\end{tabular} & $\begin{array}{l}1 \\
2 \\
\end{array}$ & 5 & & & $\begin{array}{l}1 \\
3 \\
\end{array}$ & 1 & $\begin{array}{l}1 \\
4 \\
\end{array}$ & 4 & $\begin{array}{l}1 \\
7\end{array}$ & $\begin{array}{l}1 \\
8 \\
\end{array}$ & 9 & 3 & $\begin{array}{l}1 \\
5\end{array}$ & $\begin{array}{l}1 \\
6 \\
\end{array}$ & $\begin{array}{l}1 \\
0 \\
\end{array}$ & & & $\begin{array}{l}0,912 \\
281 \\
\end{array}$ \\
\hline 19 & & 10 & 9 & 5 & 4 & 8 & 1 & & & $\begin{array}{l}1 \\
1 \\
\end{array}$ & 3 & $\begin{array}{l}1 \\
4 \\
\end{array}$ & 6 & $\begin{array}{l}1 \\
7\end{array}$ & $\begin{array}{l}1 \\
8 \\
\end{array}$ & $\begin{array}{l}1 \\
3 \\
\end{array}$ & 2 & $\begin{array}{l}1 \\
5 \\
\end{array}$ & $\begin{array}{l}1 \\
6 \\
\end{array}$ & $\begin{array}{l}1 \\
2 \\
\end{array}$ & & & $\begin{array}{l}0,895 \\
769\end{array}$ \\
\hline 20 & & 15 & $\begin{array}{l}1 \\
4 \\
\end{array}$ & 6 & 5 & 3 & 1 & & & 4 & 2 & 8 & $\begin{array}{l}1 \\
3 \\
\end{array}$ & $\begin{array}{l}1 \\
6 \\
\end{array}$ & $\begin{array}{l}1 \\
7 \\
\end{array}$ & $\begin{array}{l}1 \\
0 \\
\end{array}$ & 9 & $\begin{array}{l}1 \\
1 \\
\end{array}$ & $\begin{array}{l}1 \\
8 \\
\end{array}$ & $\begin{array}{l}1 \\
2 \\
\end{array}$ & & & $\begin{array}{l}0,830 \\
753\end{array}$ \\
\hline 21 & & 10 & $\begin{array}{l}1 \\
5 \\
\end{array}$ & 1 & 2 & 5 & 6 & & & $\begin{array}{l}1 \\
6 \\
\end{array}$ & 3 & 4 & $\begin{array}{l}1 \\
7 \\
\end{array}$ & $\begin{array}{l}1 \\
8 \\
\end{array}$ & $\begin{array}{l}1 \\
2 \\
\end{array}$ & 9 & 7 & $\begin{array}{l}1 \\
4 \\
\end{array}$ & $\begin{array}{l}1 \\
3 \\
\end{array}$ & $\begin{array}{l}1 \\
1 \\
\end{array}$ & & & $\begin{array}{l}0,805 \\
986 \\
\end{array}$ \\
\hline 22 & & 7 & $\begin{array}{l}1 \\
2 \\
\end{array}$ & 2 & 6 & 4 & 1 & & & 5 & 3 & $\begin{array}{l}1 \\
8 \\
\end{array}$ & 8 & $\begin{array}{l}1 \\
3 \\
\end{array}$ & $\begin{array}{l}1 \\
7\end{array}$ & $\begin{array}{l}1 \\
0\end{array}$ & 9 & $\begin{array}{l}1 \\
4 \\
\end{array}$ & $\begin{array}{l}1 \\
5\end{array}$ & $\begin{array}{l}1 \\
6 \\
\end{array}$ & & & $\begin{array}{l}0,835 \\
913\end{array}$ \\
\hline 23 & & 7 & $\begin{array}{l}1 \\
0 \\
\end{array}$ & 2 & 6 & 4 & 3 & & & 5 & 1 & $\begin{array}{l}1 \\
1 \\
\end{array}$ & $\begin{array}{l}1 \\
4 \\
\end{array}$ & $\begin{array}{l}1 \\
5\end{array}$ & $\begin{array}{l}1 \\
8 \\
\end{array}$ & $\begin{array}{l}1 \\
2 \\
\end{array}$ & $\begin{array}{l}1 \\
3 \\
\end{array}$ & $\begin{array}{l}1 \\
6 \\
\end{array}$ & $\begin{array}{l}1 \\
7\end{array}$ & 8 & & & $\begin{array}{l}0,819 \\
401\end{array}$ \\
\hline 24 & & 7 & 9 & 6 & 8 & $\begin{array}{l}1 \\
0 \\
\end{array}$ & 1 & & & $\begin{array}{l}1 \\
1 \\
\end{array}$ & 3 & $\begin{array}{l}1 \\
2 \\
\end{array}$ & $\begin{array}{l}1 \\
3 \\
\end{array}$ & $\begin{array}{l}1 \\
7\end{array}$ & $\begin{array}{l}1 \\
8 \\
\end{array}$ & 5 & 4 & $\begin{array}{l}1 \\
4 \\
\end{array}$ & $\begin{array}{l}1 \\
5 \\
\end{array}$ & $\begin{array}{l}1 \\
6 \\
\end{array}$ & & & $\begin{array}{l}0,995 \\
872\end{array}$ \\
\hline 25 & & 5 & $\begin{array}{l}1 \\
3 \\
\end{array}$ & 6 & \begin{tabular}{|l}
1 \\
2 \\
\end{tabular} & 4 & 2 & & & $\begin{array}{l}1 \\
1 \\
\end{array}$ & 3 & $\begin{array}{l}1 \\
0 \\
\end{array}$ & $\begin{array}{l}1 \\
8 \\
\end{array}$ & $\begin{array}{l}1 \\
4 \\
\end{array}$ & $\begin{array}{l}1 \\
7 \\
\end{array}$ & 8 & $\begin{array}{l}1 \\
5 \\
\end{array}$ & $\begin{array}{l}1 \\
6 \\
\end{array}$ & 9 & 7 & & & $\begin{array}{l}0,810 \\
113\end{array}$ \\
\hline 26 & & 5 & 3 & 4 & \begin{tabular}{|l|}
1 \\
1 \\
\end{tabular} & $\begin{array}{l}1 \\
3 \\
\end{array}$ & 1 & & & $\begin{array}{l}1 \\
2 \\
\end{array}$ & 6 & $\begin{array}{l}1 \\
5 \\
\end{array}$ & 7 & $\begin{array}{l}1 \\
4\end{array}$ & $\begin{array}{l}1 \\
8 \\
\end{array}$ & $\begin{array}{l}1 \\
0 \\
\end{array}$ & 8 & 9 & $\begin{array}{l}1 \\
7 \\
\end{array}$ & $\begin{array}{l}1 \\
6 \\
\end{array}$ & & & $\begin{array}{l}0,888 \\
545\end{array}$ \\
\hline 27 & & 8 & $\begin{array}{l}1 \\
6 \\
\end{array}$ & 2 & 3 & 5 & 7 & & & 6 & 4 & $\begin{array}{l}1 \\
0 \\
\end{array}$ & $\begin{array}{l}1 \\
7 \\
\end{array}$ & 9 & $\begin{array}{l}1 \\
8 \\
\end{array}$ & $\begin{array}{l}1 \\
1 \\
\end{array}$ & $\begin{array}{l}1 \\
4 \\
\end{array}$ & $\begin{array}{l}1 \\
3 \\
\end{array}$ & $\begin{array}{l}1 \\
5 \\
\end{array}$ & $\begin{array}{l}1 \\
2 \\
\end{array}$ & & & $\begin{array}{l}0,791 \\
538 \\
\end{array}$ \\
\hline
\end{tabular}




\begin{tabular}{|c|c|c|c|c|c|c|}
\hline \multirow{4}{*}{ Impact Factor: } & ISRA (India) & $=3.117$ & SIS (USA) & $=0.912$ & ICV (Poland) & $=6.630$ \\
\hline & ISI (Dubai, UAE & $=0.829$ & РИНЦ (Russia) & $=0.156$ & PIF (India) & $=1.940$ \\
\hline & GIF (Australia) & $=0.564$ & ESJI (KZ) & $=8.716$ & IBI (India) & $=4.260$ \\
\hline & JIF & $=1.500$ & SJIF (Morocco) & $=5.667$ & OAJI (USA) & $=0.350$ \\
\hline
\end{tabular}

\begin{tabular}{|c|c|c|c|c|c|c|c|c|c|c|c|c|c|c|c|c|c|c|c|}
\hline 28 & 13 & 6 & 1 & 5 & $\begin{array}{l}1 \\
7\end{array}$ & 2 & 3 & $\begin{array}{l}1 \\
4 \\
\end{array}$ & 4 & $\begin{array}{l}1 \\
5\end{array}$ & $\begin{array}{l}1 \\
8\end{array}$ & 7 & $\begin{array}{l}1 \\
6\end{array}$ & 9 & 8 & $\begin{array}{l}1 \\
1\end{array}$ & $\begin{array}{l}1 \\
0\end{array}$ & $\begin{array}{l}1 \\
2\end{array}$ & $\begin{array}{l}0,810 \\
113 \\
\end{array}$ \\
\hline 29 & 8 & $\begin{array}{l}1 \\
7\end{array}$ & 1 & 5 & 9 & 3 & 2 & 7 & 4 & $\begin{array}{l}1 \\
0\end{array}$ & $\begin{array}{l}1 \\
8\end{array}$ & 6 & $\begin{array}{l}1 \\
2\end{array}$ & $\begin{array}{l}1 \\
4\end{array}$ & \begin{tabular}{|l|}
1 \\
3
\end{tabular} & $\begin{array}{l}1 \\
5\end{array}$ & \begin{tabular}{l|}
1 \\
6
\end{tabular} & $\begin{array}{l}1 \\
1\end{array}$ & $\begin{array}{l}0,742 \\
002\end{array}$ \\
\hline 30 & 5 & $\begin{array}{l}1 \\
3 \\
\end{array}$ & 2 & $\begin{array}{l}1 \\
0\end{array}$ & 9 & 3 & 4 & $\begin{array}{l}1 \\
2\end{array}$ & 1 & $\begin{array}{l}1 \\
1 \\
1\end{array}$ & 8 & \begin{tabular}{l|}
1 \\
7
\end{tabular} & $\begin{array}{l}1 \\
8 \\
\end{array}$ & 7 & 6 & $\begin{array}{l}1 \\
4\end{array}$ & $\begin{array}{l}1 \\
5 \\
\end{array}$ & $\begin{array}{l}1 \\
6\end{array}$ & $\begin{array}{l}0,958 \\
72\end{array}$ \\
\hline 31 & 6 & 9 & \begin{tabular}{|l}
8 \\
\end{tabular} & 2 & 3 & 5 & 7 & $\begin{array}{l}1 \\
1 \\
\end{array}$ & 4 & $\begin{array}{l}1 \\
0 \\
\end{array}$ & $\begin{array}{l}1 \\
3 \\
\end{array}$ & 1 & $\begin{array}{l}1 \\
2 \\
\end{array}$ & $\begin{array}{l}1 \\
4 \\
\end{array}$ & $\begin{array}{l}1 \\
6 \\
\end{array}$ & $\begin{array}{l}1 \\
7 \\
\end{array}$ & \begin{tabular}{|l|}
1 \\
5 \\
\end{tabular} & $\begin{array}{l}1 \\
8 \\
\end{array}$ & $\begin{array}{l}0,658 \\
411 \\
\end{array}$ \\
\hline 32 & 2 & 4 & 1 & 5 & 7 & 3 & 8 & $\begin{array}{l}1 \\
1\end{array}$ & 6 & $\begin{array}{l}1 \\
2\end{array}$ & 9 & $\begin{array}{l}1 \\
0\end{array}$ & $\begin{array}{l}1 \\
3 \\
\end{array}$ & $\begin{array}{l}1 \\
5\end{array}$ & $\begin{array}{l}1 \\
4 \\
\end{array}$ & $\begin{array}{l}1 \\
7\end{array}$ & $\begin{array}{l}1 \\
6 \\
\end{array}$ & $\begin{array}{l}1 \\
8\end{array}$ & $\begin{array}{l}0,763 \\
674\end{array}$ \\
\hline 33 & 11 & 4 & 3 & $\begin{array}{l}1 \\
0 \\
\end{array}$ & \begin{tabular}{l|}
1 \\
2 \\
\end{tabular} & $\begin{array}{l}1 \\
3 \\
\end{array}$ & 2 & 1 & 9 & 8 & $\begin{array}{l}1 \\
5 \\
\end{array}$ & \begin{tabular}{|l|}
1 \\
4 \\
\end{tabular} & $\begin{array}{l}1 \\
7 \\
\end{array}$ & $\begin{array}{l}1 \\
6 \\
\end{array}$ & \begin{tabular}{|l|}
6 \\
\end{tabular} & 5 & 7 & $\begin{array}{l}1 \\
8 \\
\end{array}$ & $\begin{array}{l}0,676 \\
987\end{array}$ \\
\hline 34 & 18 & \begin{tabular}{|l|}
1 \\
7
\end{tabular} & 1 & 2 & 5 & 3 & 4 & 6 & 7 & $\begin{array}{l}1 \\
0\end{array}$ & $\begin{array}{l}1 \\
1\end{array}$ & 8 & $\begin{array}{l}1 \\
2\end{array}$ & $\begin{array}{l}1 \\
6\end{array}$ & \begin{tabular}{|l|}
1 \\
4
\end{tabular} & $\begin{array}{l}1 \\
3\end{array}$ & $\begin{array}{l}1 \\
5 \\
\end{array}$ & 9 & $\begin{array}{l}0,622 \\
291\end{array}$ \\
\hline 35 & 1 & 9 & \begin{tabular}{|l|}
4 \\
\end{tabular} & 3 & 8 & $\begin{array}{l}1 \\
0\end{array}$ & 5 & 6 & 2 & $\begin{array}{l}1 \\
1\end{array}$ & $\begin{array}{l}1 \\
3\end{array}$ & $\begin{array}{l}1 \\
2\end{array}$ & $\begin{array}{l}1 \\
5\end{array}$ & $\begin{array}{l}1 \\
4\end{array}$ & \begin{tabular}{|l|}
1 \\
6 \\
\end{tabular} & $\begin{array}{l}1 \\
8\end{array}$ & $\begin{array}{l}1 \\
7 \\
\end{array}$ & 7 & $\begin{array}{l}0,734 \\
778\end{array}$ \\
\hline 36 & 4 & 3 & 5 & 7 & 2 & 1 & 6 & $\begin{array}{l}1 \\
2\end{array}$ & 8 & 9 & $\begin{array}{l}1 \\
0\end{array}$ & $\begin{array}{l}1 \\
3\end{array}$ & $\begin{array}{l}1 \\
5\end{array}$ & $\begin{array}{l}1 \\
4\end{array}$ & $\begin{array}{l}1 \\
8\end{array}$ & $\begin{array}{l}1 \\
7\end{array}$ & $\begin{array}{l}1 \\
6\end{array}$ & $\begin{array}{l}1 \\
1\end{array}$ & $\begin{array}{l}0,723 \\
426\end{array}$ \\
\hline 37 & 8 & \begin{tabular}{|l|}
1 \\
3
\end{tabular} & 4 & 3 & 9 & 1 & $\begin{array}{l}1 \\
0\end{array}$ & $\begin{array}{l}1 \\
2\end{array}$ & 2 & 5 & $\begin{array}{l}1 \\
4\end{array}$ & 6 & 7 & $\begin{array}{l}1 \\
5\end{array}$ & \begin{tabular}{|l}
1 \\
6
\end{tabular} & $\begin{array}{l}1 \\
7\end{array}$ & $\begin{array}{l}1 \\
8 \\
\end{array}$ & $\begin{array}{l}1 \\
1\end{array}$ & $\begin{array}{l}0,640 \\
867\end{array}$ \\
\hline 38 & 13 & $\begin{array}{l}1 \\
1 \\
\end{array}$ & \begin{tabular}{|l}
9 \\
\end{tabular} & 1 & 2 & 6 & 3 & 5 & 7 & 4 & $\begin{array}{l}1 \\
2 \\
\end{array}$ & \begin{tabular}{|l|}
1 \\
4 \\
\end{tabular} & 8 & $\begin{array}{l}1 \\
7 \\
\end{array}$ & $\begin{array}{l}1 \\
5 \\
\end{array}$ & $\begin{array}{l}1 \\
8 \\
\end{array}$ & \begin{tabular}{|l|}
1 \\
6 \\
\end{tabular} & $\begin{array}{l}1 \\
0\end{array}$ & $\begin{array}{l}0,620 \\
227 \\
\end{array}$ \\
\hline 39 & 4 & $\begin{array}{l}1 \\
8\end{array}$ & 5 & $\begin{array}{l}1 \\
7\end{array}$ & 1 & $\begin{array}{l}1 \\
6\end{array}$ & 3 & $\begin{array}{l}1 \\
3\end{array}$ & 2 & $\begin{array}{l}1 \\
2\end{array}$ & $\begin{array}{l}1 \\
1\end{array}$ & $\begin{array}{l}1 \\
5\end{array}$ & $\begin{array}{l}1 \\
4 \\
\end{array}$ & 8 & 7 & 9 & $\begin{array}{l}1 \\
0\end{array}$ & 6 & $\begin{array}{l}0,680 \\
083 \\
\end{array}$ \\
\hline 40 & 5 & \begin{tabular}{|l|}
1 \\
3 \\
\end{tabular} & 2 & $\begin{array}{l}1 \\
0 \\
\end{array}$ & 9 & 3 & 4 & $\begin{array}{l}1 \\
2 \\
\end{array}$ & 1 & $\begin{array}{l}1 \\
1 \\
\end{array}$ & 8 & \begin{tabular}{|l|}
1 \\
7 \\
\end{tabular} & $\begin{array}{l}1 \\
8 \\
\end{array}$ & 7 & 6 & $\begin{array}{l}1 \\
4 \\
\end{array}$ & $\begin{array}{l}1 \\
5 \\
\end{array}$ & $\begin{array}{l}1 \\
6 \\
\end{array}$ & $\begin{array}{l}0,958 \\
72\end{array}$ \\
\hline 41 & 13 & 6 & 1 & 5 & $\begin{array}{l}1 \\
7\end{array}$ & 2 & 3 & \begin{tabular}{|l|}
1 \\
4 \\
\end{tabular} & 4 & $\begin{array}{l}1 \\
5\end{array}$ & 7 & $\begin{array}{l}1 \\
6\end{array}$ & 9 & 8 & \begin{tabular}{|l|}
1 \\
1
\end{tabular} & $\begin{array}{l}1 \\
0\end{array}$ & $\begin{array}{l}1 \\
2 \\
\end{array}$ & $\begin{array}{l}1 \\
8\end{array}$ & $\begin{array}{l}0,813 \\
209\end{array}$ \\
\hline 42 & 8 & \begin{tabular}{|l|}
1 \\
6 \\
\end{tabular} & 2 & 3 & 5 & 7 & 1 & 6 & 4 & $\begin{array}{l}1 \\
0\end{array}$ & $\begin{array}{l}1 \\
7\end{array}$ & 9 & $\begin{array}{l}1 \\
8\end{array}$ & $\begin{array}{l}1 \\
1\end{array}$ & \begin{tabular}{|l|}
1 \\
4
\end{tabular} & $\begin{array}{l}1 \\
3\end{array}$ & $\begin{array}{l}1 \\
5\end{array}$ & $\begin{array}{l}1 \\
2\end{array}$ & $\begin{array}{l}0,791 \\
538\end{array}$ \\
\hline 43 & 5 & 3 & 4 & $\begin{array}{l}1 \\
1\end{array}$ & $\begin{array}{l}1 \\
3\end{array}$ & 1 & 2 & $\begin{array}{l}1 \\
2\end{array}$ & 6 & $\begin{array}{l}1 \\
5\end{array}$ & 7 & $\begin{array}{l}1 \\
4\end{array}$ & $\begin{array}{l}1 \\
8\end{array}$ & $\begin{array}{l}1 \\
0\end{array}$ & \begin{tabular}{|l|}
8 \\
\end{tabular} & 9 & $\begin{array}{l}1 \\
7\end{array}$ & $\begin{array}{l}1 \\
6\end{array}$ & $\begin{array}{l}0,888 \\
545\end{array}$ \\
\hline 44 & 5 & $\begin{array}{l}1 \\
3 \\
\end{array}$ & \begin{tabular}{|l|}
6 \\
\end{tabular} & $\begin{array}{l}1 \\
2 \\
\end{array}$ & 4 & 2 & 1 & $\begin{array}{l}1 \\
1 \\
\end{array}$ & 3 & $\begin{array}{l}1 \\
0 \\
\end{array}$ & $\begin{array}{l}1 \\
8 \\
\end{array}$ & $\begin{array}{l}1 \\
4 \\
\end{array}$ & $\begin{array}{l}1 \\
7 \\
\end{array}$ & 8 & $\begin{array}{l}1 \\
5 \\
\end{array}$ & $\begin{array}{l}1 \\
6 \\
\end{array}$ & 9 & 7 & $\begin{array}{l}0,810 \\
113\end{array}$ \\
\hline 45 & 7 & 9 & 6 & 8 & $\begin{array}{l}1 \\
0\end{array}$ & 1 & 2 & $\begin{array}{l}1 \\
1\end{array}$ & 3 & $\begin{array}{l}1 \\
2\end{array}$ & $\begin{array}{l}1 \\
3\end{array}$ & $\begin{array}{l}1 \\
7\end{array}$ & $\begin{array}{l}1 \\
8\end{array}$ & 5 & 4 & $\begin{array}{l}1 \\
4\end{array}$ & $\begin{array}{l}1 \\
5\end{array}$ & $\begin{array}{l}1 \\
6\end{array}$ & $\begin{array}{l}0,995 \\
872\end{array}$ \\
\hline 46 & 7 & \begin{tabular}{|l|}
1 \\
0
\end{tabular} & 2 & 6 & 4 & 3 & 9 & 5 & 1 & $\begin{array}{l}1 \\
1\end{array}$ & $\begin{array}{l}1 \\
4\end{array}$ & $\begin{array}{l}1 \\
5\end{array}$ & $\begin{array}{l}1 \\
8 \\
\end{array}$ & $\begin{array}{l}1 \\
2\end{array}$ & \begin{tabular}{|l|}
1 \\
3 \\
\end{tabular} & $\begin{array}{l}1 \\
6\end{array}$ & $\begin{array}{l}1 \\
7\end{array}$ & 8 & $\begin{array}{l}0,819 \\
401\end{array}$ \\
\hline 47 & 7 & \begin{tabular}{|l|}
1 \\
2 \\
\end{tabular} & 2 & 6 & 4 & 1 & $\begin{array}{l}1 \\
1 \\
\end{array}$ & 5 & 3 & \begin{tabular}{|l|}
1 \\
8 \\
\end{tabular} & 8 & \begin{tabular}{|l|}
1 \\
3 \\
\end{tabular} & $\begin{array}{l}1 \\
7 \\
\end{array}$ & $\begin{array}{l}1 \\
0 \\
\end{array}$ & \begin{tabular}{|l|}
9 \\
\end{tabular} & $\begin{array}{l}1 \\
4 \\
\end{array}$ & $\begin{array}{l}1 \\
5 \\
\end{array}$ & $\begin{array}{l}1 \\
6 \\
\end{array}$ & $\begin{array}{l}0,835 \\
913\end{array}$ \\
\hline 48 & 10 & \begin{tabular}{|l|}
1 \\
5
\end{tabular} & 1 & 2 & \begin{tabular}{|l|}
5 \\
\end{tabular} & 6 & 8 & $\begin{array}{l}1 \\
6 \\
\end{array}$ & 3 & 4 & $\begin{array}{l}1 \\
7\end{array}$ & $\begin{array}{l}1 \\
8\end{array}$ & $\begin{array}{l}1 \\
2\end{array}$ & 9 & 7 & $\begin{array}{l}1 \\
4\end{array}$ & $\begin{array}{l}1 \\
3\end{array}$ & $\begin{array}{l}1 \\
1 \\
1\end{array}$ & $\begin{array}{l}0,805 \\
986\end{array}$ \\
\hline 49 & 15 & \begin{tabular}{|l}
1 \\
4
\end{tabular} & 6 & 5 & 3 & 1 & 7 & 4 & 2 & 8 & $\begin{array}{l}1 \\
3\end{array}$ & $\begin{array}{l}1 \\
6\end{array}$ & $\begin{array}{l}1 \\
7\end{array}$ & $\begin{array}{l}1 \\
0\end{array}$ & \begin{tabular}{|l|}
9 \\
\end{tabular} & $\begin{array}{l}1 \\
1\end{array}$ & $\begin{array}{l}1 \\
8\end{array}$ & $\begin{array}{l}1 \\
2 \\
\end{array}$ & $\begin{array}{l}0,830 \\
753\end{array}$ \\
\hline 50 & 10 & 9 & 5 & 4 & 8 & 1 & 7 & $\begin{array}{l}1 \\
1\end{array}$ & 3 & $\begin{array}{l}1 \\
4\end{array}$ & 6 & $\begin{array}{l}1 \\
7\end{array}$ & $\begin{array}{l}1 \\
8\end{array}$ & $\begin{array}{l}1 \\
3\end{array}$ & 2 & $\begin{array}{l}1 \\
5\end{array}$ & $\begin{array}{l}1 \\
6\end{array}$ & $\begin{array}{l}1 \\
2 \\
\end{array}$ & $\begin{array}{l}0,895 \\
769\end{array}$ \\
\hline 51 & 6 & 7 & \begin{tabular}{|l|}
8 \\
\end{tabular} & $\begin{array}{l}1 \\
1 \\
\end{array}$ & \begin{tabular}{|l}
1 \\
2 \\
\end{tabular} & 5 & 2 & $\begin{array}{l}1 \\
3 \\
\end{array}$ & 1 & $\begin{array}{l}1 \\
4 \\
\end{array}$ & 4 & $\begin{array}{l}1 \\
7 \\
\end{array}$ & $\begin{array}{l}1 \\
8 \\
\end{array}$ & 9 & 3 & $\begin{array}{l}1 \\
5 \\
\end{array}$ & $\begin{array}{l}1 \\
6 \\
\end{array}$ & $\begin{array}{l}1 \\
0 \\
\end{array}$ & $\begin{array}{l}0,912 \\
281 \\
\end{array}$ \\
\hline 52 & 5 & \begin{tabular}{|l|}
1 \\
3
\end{tabular} & 1 & 6 & $\begin{array}{l}1 \\
1\end{array}$ & 2 & 3 & $\begin{array}{l}1 \\
2\end{array}$ & 4 & $\begin{array}{l}1 \\
8\end{array}$ & 9 & $\begin{array}{l}1 \\
0\end{array}$ & $\begin{array}{l}1 \\
6\end{array}$ & $\begin{array}{l}1 \\
5\end{array}$ & 7 & $\begin{array}{l}1 \\
4\end{array}$ & $\begin{array}{l}1 \\
7\end{array}$ & 8 & $\begin{array}{l}0,822 \\
497\end{array}$ \\
\hline 53 & 4 & \begin{tabular}{|l|}
1 \\
1 \\
\end{tabular} & \begin{tabular}{|l|}
3 \\
\end{tabular} & $\begin{array}{l}1 \\
0 \\
\end{array}$ & \begin{tabular}{|l|}
1 \\
6 \\
\end{tabular} & 1 & 9 & $\begin{array}{l}1 \\
5 \\
\end{array}$ & 2 & $\begin{array}{l}1 \\
7\end{array}$ & 5 & $\begin{array}{l}1 \\
4\end{array}$ & $\begin{array}{l}1 \\
8\end{array}$ & 7 & 6 & $\begin{array}{l}1 \\
2\end{array}$ & $\begin{array}{l}1 \\
3\end{array}$ & 8 & $\begin{array}{l}0,840 \\
041\end{array}$ \\
\hline 54 & 7 & 9 & 6 & 8 & $\begin{array}{l}1 \\
0\end{array}$ & 3 & 2 & $\begin{array}{l}1 \\
1\end{array}$ & 1 & $\begin{array}{l}1 \\
2\end{array}$ & $\begin{array}{l}1 \\
3\end{array}$ & $\begin{array}{l}1 \\
7\end{array}$ & $\begin{array}{l}1 \\
8\end{array}$ & 5 & 4 & $\begin{array}{l}1 \\
4\end{array}$ & $\begin{array}{l}1 \\
5\end{array}$ & $\begin{array}{l}1 \\
6\end{array}$ & 0,996 \\
\hline
\end{tabular}

Table 7 - Results of assessment of competence of manufacturers who participated in the survey to assess the competitiveness and demand for children's shoes 


\begin{tabular}{|c|c|c|c|c|c|c|}
\hline \multirow{4}{*}{ Impact Factor: } & ISRA (India) & $=3.117$ & SIS (USA) & $=0.912$ & ICV (Poland) & $=6.630$ \\
\hline & ISI (Dubai, UAE & $=0.829$ & РИНЦ (Russia) & $=0.156$ & PIF (India) & $=1.940$ \\
\hline & GIF (Australia) & $=0.564$ & ESJI (KZ) & $=8.716$ & IBI (India) & $=4.260$ \\
\hline & JIF & $=1.500$ & SJIF (Morocco) & $=5.667$ & OAJI (USA) & $=0.350$ \\
\hline
\end{tabular}

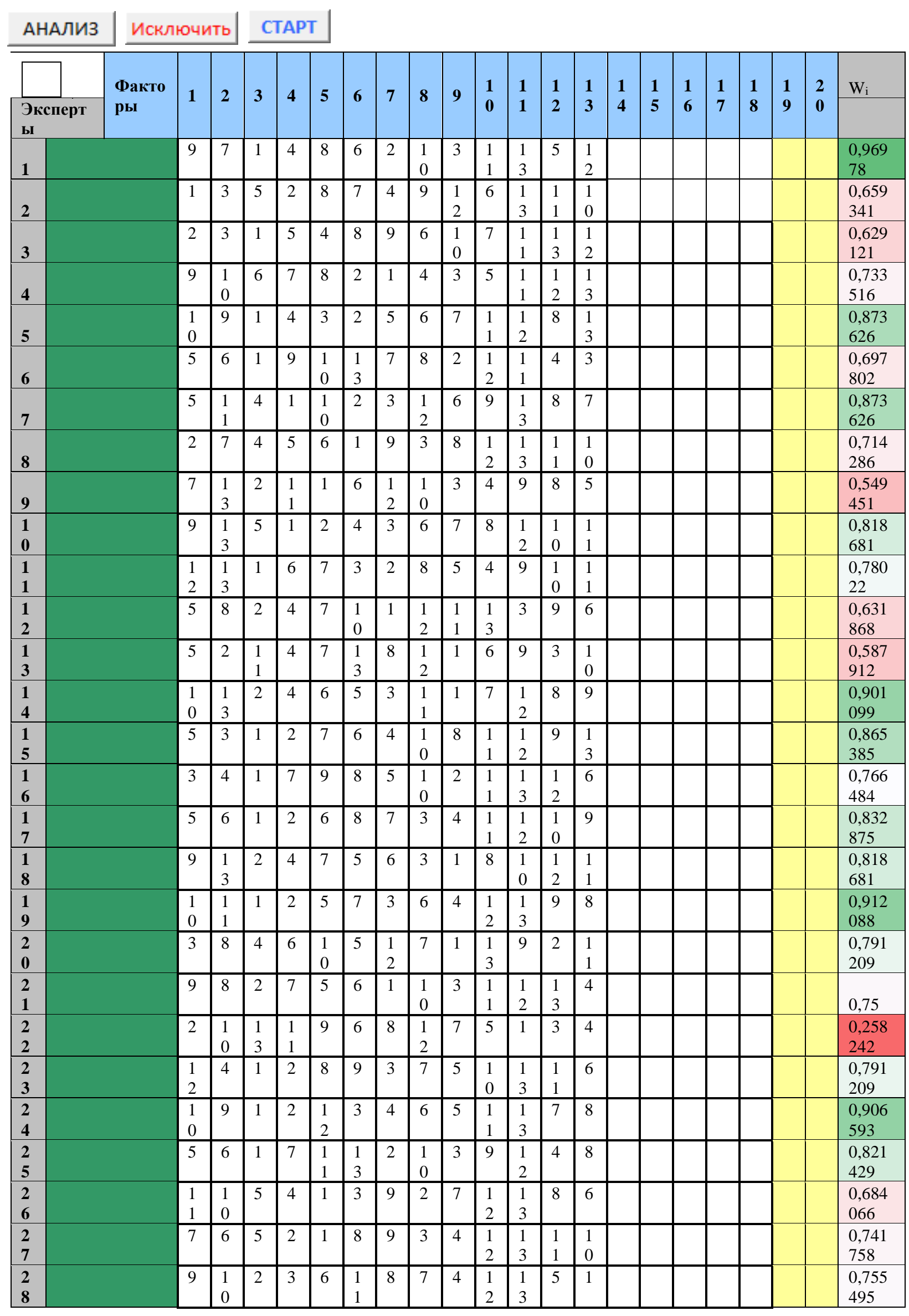




\begin{tabular}{|c|c|c|c|c|c|c|}
\hline \multirow{4}{*}{ Impact Factor: } & ISRA (India) & $=3.117$ & SIS (USA) & $=0.912$ & ICV (Poland) & $=6.630$ \\
\hline & ISI (Dubai, UAE & $=0.829$ & РИНЦ (Russia & $=0.156$ & PIF (India) & $=1.940$ \\
\hline & GIF (Australia) & $=0.564$ & ESJI (KZ) & $=8.716$ & IBI (India) & $=4.260$ \\
\hline & JIF & $=1.500$ & SJIF (Morocce & $=5.667$ & OAJI (USA) & $=0.350$ \\
\hline
\end{tabular}

\begin{tabular}{|l|l|l|l|l|l|l|l|l|l|l|l|l|l|l|l|l|l|l|l|l|l|}
$\mathbf{2}$ \\
$\mathbf{9}$
\end{tabular}

Table 8 - The results of evaluation of competence of parents who participated in the survey on the assessment competitiveness and demand for children's shoes

\begin{tabular}{|c|c|c|c|c|c|c|c|c|c|c|c|c|c|c|c|c|c|c|c|c|c|c|}
\hline Al & - Искл & рчи & & & CTA & & & & & & & & & & & & & & & & & \\
\hline & Факто & 1 & 2 & 3 & 4 & 5 & 6 & 7 & 8 & 9 & $\begin{array}{l}1 \\
0\end{array}$ & 1 & $\begin{array}{l}1 \\
2\end{array}$ & $\begin{array}{l}1 \\
3\end{array}$ & $\begin{array}{l}1 \\
4\end{array}$ & $\begin{array}{l}1 \\
5\end{array}$ & $\begin{array}{l}1 \\
6\end{array}$ & $\begin{array}{l}1 \\
7\end{array}$ & $\begin{array}{l}1 \\
8\end{array}$ & $\begin{array}{l}1 \\
9\end{array}$ & $\begin{array}{l}2 \\
0\end{array}$ & $\mathrm{~W}_{\mathrm{i}}$ \\
\hline Экс & & & & & & & & & & & & & & & & & & & & & & \\
\hline 1 & & 4 & $\begin{array}{l}1 \\
1\end{array}$ & 2 & 3 & 7 & 5 & 6 & 1 & 8 & \begin{tabular}{|l|}
1 \\
0 \\
\end{tabular} & 9 & & & & & & & & & & $\begin{array}{l}0,836 \\
364\end{array}$ \\
\hline 2 & & 3 & 4 & 1 & 7 & 8 & 6 & 5 & 2 & \begin{tabular}{|l|}
1 \\
1
\end{tabular} & \begin{tabular}{|l|}
1 \\
0
\end{tabular} & 9 & & & & & & & & & & $\begin{array}{l}0,877 \\
273\end{array}$ \\
\hline 3 & & 3 & 4 & 1 & 5 & 6 & 8 & 7 & 2 & \begin{tabular}{|l}
1 \\
0
\end{tabular} & \begin{tabular}{|l|}
9 \\
\end{tabular} & \begin{tabular}{|l|}
1 \\
1
\end{tabular} & & & & & & & & & & $\begin{array}{l}0,859 \\
091\end{array}$ \\
\hline 4 & & 2 & 6 & 1 & 7 & 4 & $\begin{array}{l}1 \\
1\end{array}$ & 5 & 3 & \begin{tabular}{|l|l}
9 \\
\end{tabular} & $\begin{array}{l}1 \\
0 \\
\end{array}$ & \begin{tabular}{|l|l}
8 \\
\end{tabular} & & & & & & & & & & 0,9 \\
\hline 5 & & 4 & 8 & 1 & 5 & 7 & 9 & 3 & 2 & \begin{tabular}{|l}
1 \\
0 \\
\end{tabular} & \begin{tabular}{|l}
1 \\
1 \\
\end{tabular} & 6 & & & & & & & & & & $\begin{array}{l}0,913 \\
636 \\
\end{array}$ \\
\hline 6 & & 4 & 8 & 1 & 7 & 6 & 5 & 3 & 2 & \begin{tabular}{|l|}
1 \\
1
\end{tabular} & \begin{tabular}{|l|}
1 \\
0
\end{tabular} & 9 & & & & & & & & & & $\begin{array}{l}0,927 \\
273\end{array}$ \\
\hline 7 & & 3 & 5 & 1 & 8 & 6 & 9 & 2 & 4 & \begin{tabular}{|l|}
1 \\
1
\end{tabular} & \begin{tabular}{|l|}
7 \\
\end{tabular} & \begin{tabular}{|l|}
1 \\
0
\end{tabular} & & & & & & & & & & 0,9 \\
\hline 8 & & 2 & 3 & 4 & \begin{tabular}{|l|}
1 \\
0 \\
\end{tabular} & 5 & \begin{tabular}{|l|}
8 \\
\end{tabular} & 9 & 1 & \begin{tabular}{|l}
1 \\
1 \\
\end{tabular} & 6 & $\begin{array}{l}7 \\
\end{array}$ & & & & & & & & & & $\begin{array}{l}0,690 \\
909\end{array}$ \\
\hline 9 & & 3 & 4 & 1 & 7 & 2 & 6 & 5 & \begin{tabular}{|l|}
1 \\
0
\end{tabular} & \begin{tabular}{|l|}
1 \\
1
\end{tabular} & \begin{tabular}{|l|}
8 \\
\end{tabular} & \begin{tabular}{|l|}
9 \\
\end{tabular} & & & & & & & & & & $\begin{array}{l}0,713 \\
636\end{array}$ \\
\hline 10 & & 2 & 8 & 1 & 7 & 3 & 5 & 6 & 4 & \begin{tabular}{|l|}
1 \\
0
\end{tabular} & \begin{tabular}{|l}
9 \\
\end{tabular} & \begin{tabular}{|l|}
1 \\
1
\end{tabular} & & & & & & & & & & $\begin{array}{l}0,863 \\
636\end{array}$ \\
\hline 11 & & 3 & 7 & 1 & 6 & 5 & 8 & 4 & 2 & \begin{tabular}{|l}
1 \\
0
\end{tabular} & 9 & \begin{tabular}{|l|}
1 \\
1
\end{tabular} & & & & & & & & & & $\begin{array}{l}0,945 \\
455\end{array}$ \\
\hline 12 & & 2 & 6 & 3 & 5 & 7 & 9 & 4 & 1 & \begin{tabular}{|l}
1 \\
1 \\
\end{tabular} & 8 & \begin{tabular}{|l}
1 \\
0 \\
\end{tabular} & & & & & & & & & & $\begin{array}{l}0,927 \\
273 \\
\end{array}$ \\
\hline 13 & & 4 & 6 & 3 & 5 & 7 & \begin{tabular}{|l|}
1 \\
0 \\
\end{tabular} & 1 & 2 & \begin{tabular}{|l|}
1 \\
1 \\
\end{tabular} & 8 & \begin{tabular}{|l|l}
9 \\
\end{tabular} & & & & & & & & & & $\begin{array}{l}0,940 \\
909\end{array}$ \\
\hline 14 & & 4 & 7 & 3 & 6 & 5 & $\begin{array}{l}1 \\
0\end{array}$ & 1 & 2 & $\begin{array}{l}1 \\
1\end{array}$ & 8 & 9 & & & & & & & & & & $\begin{array}{l}0,954 \\
545\end{array}$ \\
\hline 15 & & 3 & 8 & 4 & 6 & 5 & 7 & 1 & 2 & \begin{tabular}{|l|}
1 \\
1
\end{tabular} & \begin{tabular}{|l|}
1 \\
0
\end{tabular} & 9 & & & & & & & & & & $\begin{array}{l}0,981 \\
818\end{array}$ \\
\hline 16 & & 2 & 5 & 4 & 6 & 7 & $\begin{array}{l}1 \\
0\end{array}$ & 3 & 1 & \begin{tabular}{|l|}
1 \\
1
\end{tabular} & \begin{tabular}{|l|}
9 \\
\end{tabular} & 8 & & & & & & & & & & $\begin{array}{l}0,931 \\
818\end{array}$ \\
\hline 17 & & \begin{tabular}{|l|}
5 \\
\end{tabular} & 9 & 2 & 8 & 6 & 4 & 1 & 3 & \begin{tabular}{|l|}
1 \\
0
\end{tabular} & \begin{tabular}{|l|}
1 \\
1
\end{tabular} & \begin{tabular}{|l|}
7 \\
\end{tabular} & & & & & & & & & & 0,9 \\
\hline 18 & & \begin{tabular}{|l|}
3 \\
\end{tabular} & \begin{tabular}{|l|}
7 \\
\end{tabular} & 2 & 8 & 4 & 9 & 6 & 1 & \begin{tabular}{|l|}
1 \\
0
\end{tabular} & \begin{tabular}{|l|}
1 \\
1
\end{tabular} & 5 & & & & & & & & & & $\begin{array}{l}0,859 \\
091\end{array}$ \\
\hline 19 & & \begin{tabular}{|l|}
6 \\
\end{tabular} & 5 & 1 & 8 & 4 & 7 & 3 & 2 & \begin{tabular}{|l|l}
9 \\
\end{tabular} & \begin{tabular}{|l}
1 \\
0 \\
\end{tabular} & \begin{tabular}{|l}
1 \\
1 \\
\end{tabular} & & & & & & & & & & $\begin{array}{l}0,922 \\
727 \\
\end{array}$ \\
\hline 20 & & \begin{tabular}{|l|}
3 \\
\end{tabular} & \begin{tabular}{|l|}
7 \\
\end{tabular} & 4 & 6 & 5 & 8 & 2 & 1 & 9 & $\begin{array}{l}1 \\
0 \\
\end{array}$ & \begin{tabular}{|l}
1 \\
1 \\
\end{tabular} & & & & & & & & & & $\begin{array}{l}0,990 \\
909\end{array}$ \\
\hline 21 & & \begin{tabular}{|l|}
1 \\
\end{tabular} & 3 & 5 & 4 & \begin{tabular}{|l|}
8 \\
\end{tabular} & $\begin{array}{ll}7 \\
\end{array}$ & 9 & \begin{tabular}{|l|}
1 \\
0
\end{tabular} & 2 & \begin{tabular}{|l|}
1 \\
1
\end{tabular} & 6 & & & & & & & & & & $\begin{array}{l}0,481 \\
818\end{array}$ \\
\hline 22 & & \begin{tabular}{|l|}
9 \\
\end{tabular} & $\begin{array}{l}1 \\
0\end{array}$ & 1 & 8 & 4 & 3 & 5 & 2 & \begin{tabular}{|l|}
1 \\
1
\end{tabular} & 6 & \begin{tabular}{|l|l}
7 \\
\end{tabular} & & & & & & & & & & $\begin{array}{l}0,686 \\
364\end{array}$ \\
\hline 23 & & \begin{tabular}{|l|}
4 \\
\end{tabular} & 2 & 3 & 1 & 6 & 7 & 5 & 8 & \begin{tabular}{|l|}
1 \\
1
\end{tabular} & \begin{tabular}{|l|}
1 \\
0
\end{tabular} & 9 & & & & & & & & & & $\begin{array}{l}0,745 \\
455\end{array}$ \\
\hline 24 & & 5 & $\begin{array}{l}1 \\
1\end{array}$ & 1 & 4 & 2 & 3 & \begin{tabular}{|l|}
1 \\
0
\end{tabular} & 6 & \begin{tabular}{|l}
7 \\
\end{tabular} & \begin{tabular}{|l}
9 \\
\end{tabular} & 8 & & & & & & & & & & 0,6 \\
\hline 25 & & \begin{tabular}{|l|}
1 \\
\end{tabular} & \begin{tabular}{|l|}
7 \\
\end{tabular} & 6 & 8 & 5 & 9 & \begin{tabular}{|l|}
1 \\
0
\end{tabular} & 2 & $\begin{array}{l}1 \\
1\end{array}$ & 3 & 4 & & & & & & & & & & \\
\hline
\end{tabular}

Philadelphia, USA 


\begin{tabular}{|c|c|c|c|c|c|c|}
\hline \multirow{4}{*}{ Impact Factor: } & ISRA (India) & $=3.117$ & SIS (USA) & $=0.912$ & ICV (Poland) & $=6.630$ \\
\hline & ISI (Dubai, UAE & $=0.829$ & РИНЦ (Russia & $=0.156$ & PIF (India) & $=1.940$ \\
\hline & GIF (Australia) & $=0.564$ & ESJI (KZ) & $=8.716$ & IBI (India) & $=4.260$ \\
\hline & JIF & $=1.500$ & SJIF (Morocce & $=5.667$ & OAJI (USA) & $=0.350$ \\
\hline
\end{tabular}

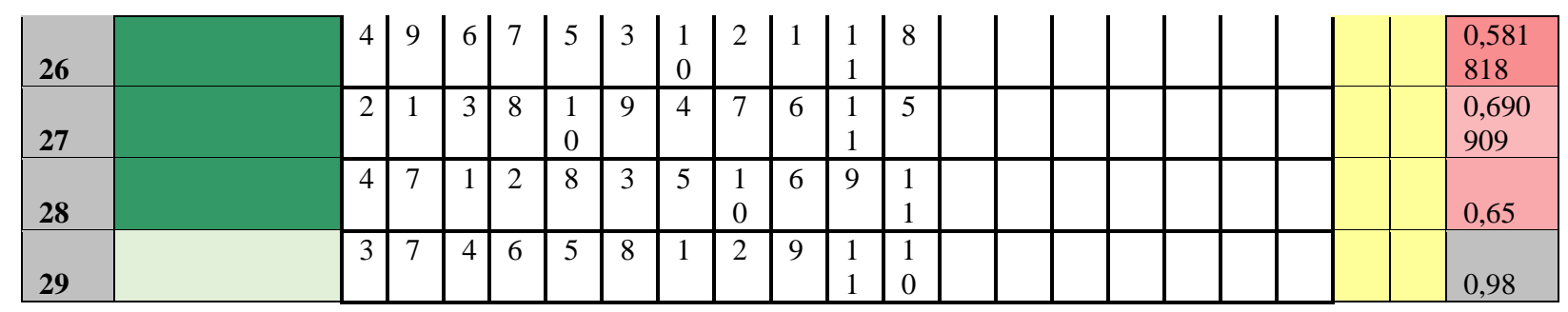

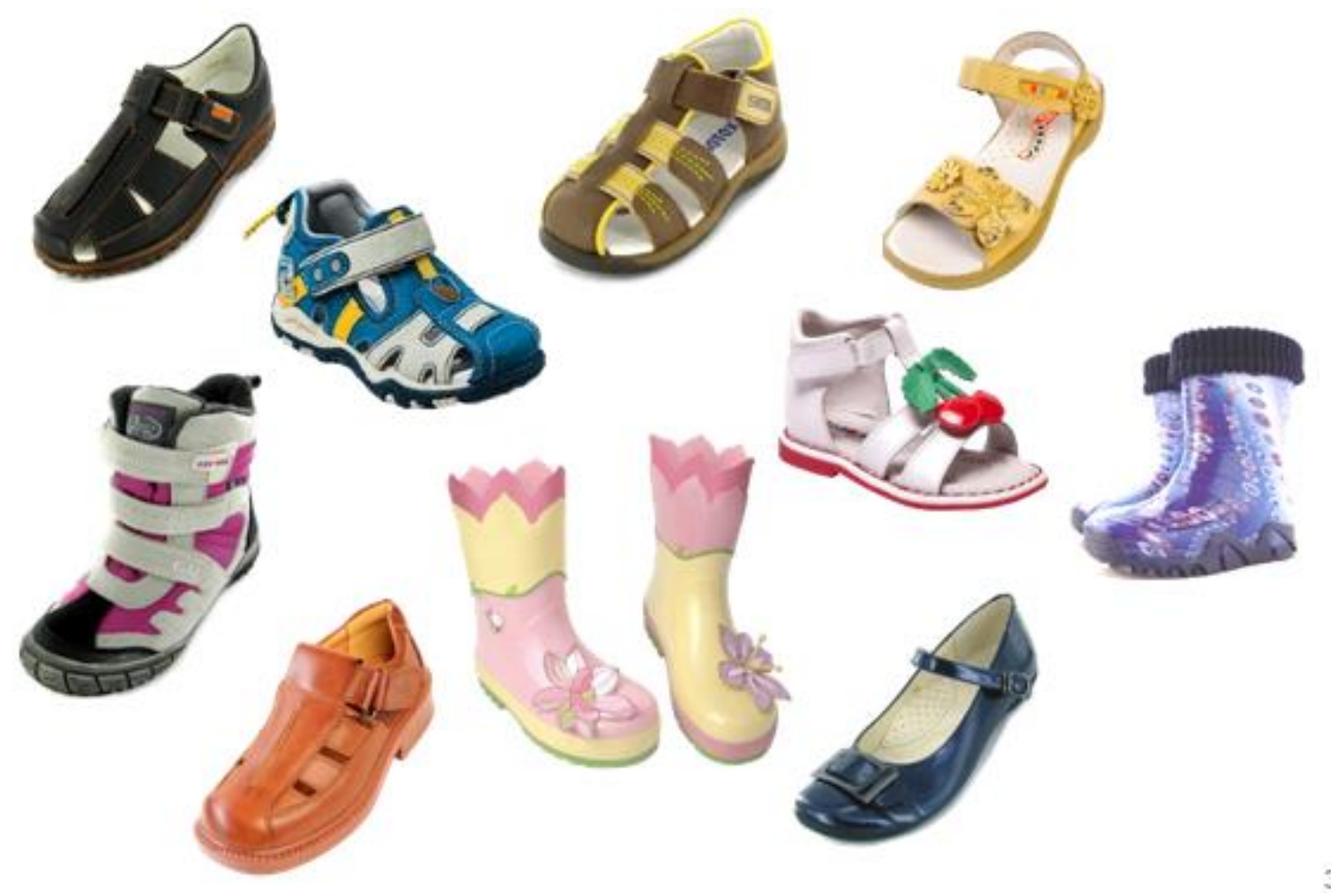

Figure 1 The range of summer children's shoes 


\begin{tabular}{|c|c|c|c|c|c|c|}
\hline \multirow{4}{*}{ Impact Factor: } & ISRA (India) & $=3.117$ & SIS (USA) & $=0.912$ & ICV (Poland) & $=6.630$ \\
\hline & ISI (Dubai, UAE & $=0.829$ & РИНЦ (Russia) & $=0.156$ & PIF (India) & $=1.940$ \\
\hline & GIF (Australia) & $=0.564$ & ESJI (KZ) & $=8.716$ & IBI (India) & $=4.260$ \\
\hline & JIF & $=1.500$ & SJIF (Morocco) & $=5.667$ & OAJI (USA) & $=0.350$ \\
\hline
\end{tabular}
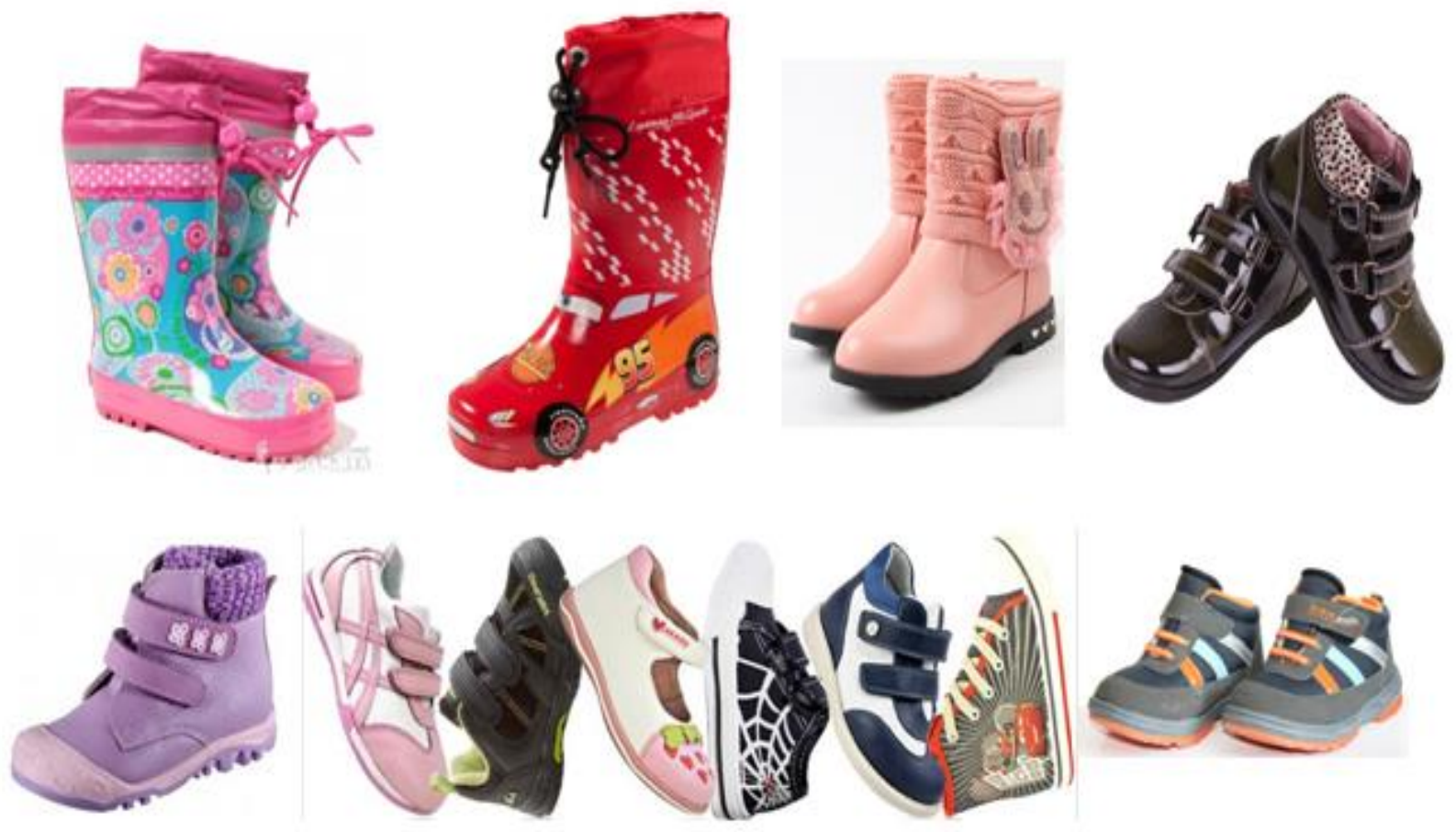

Figure 2 The range of winter children's shoes

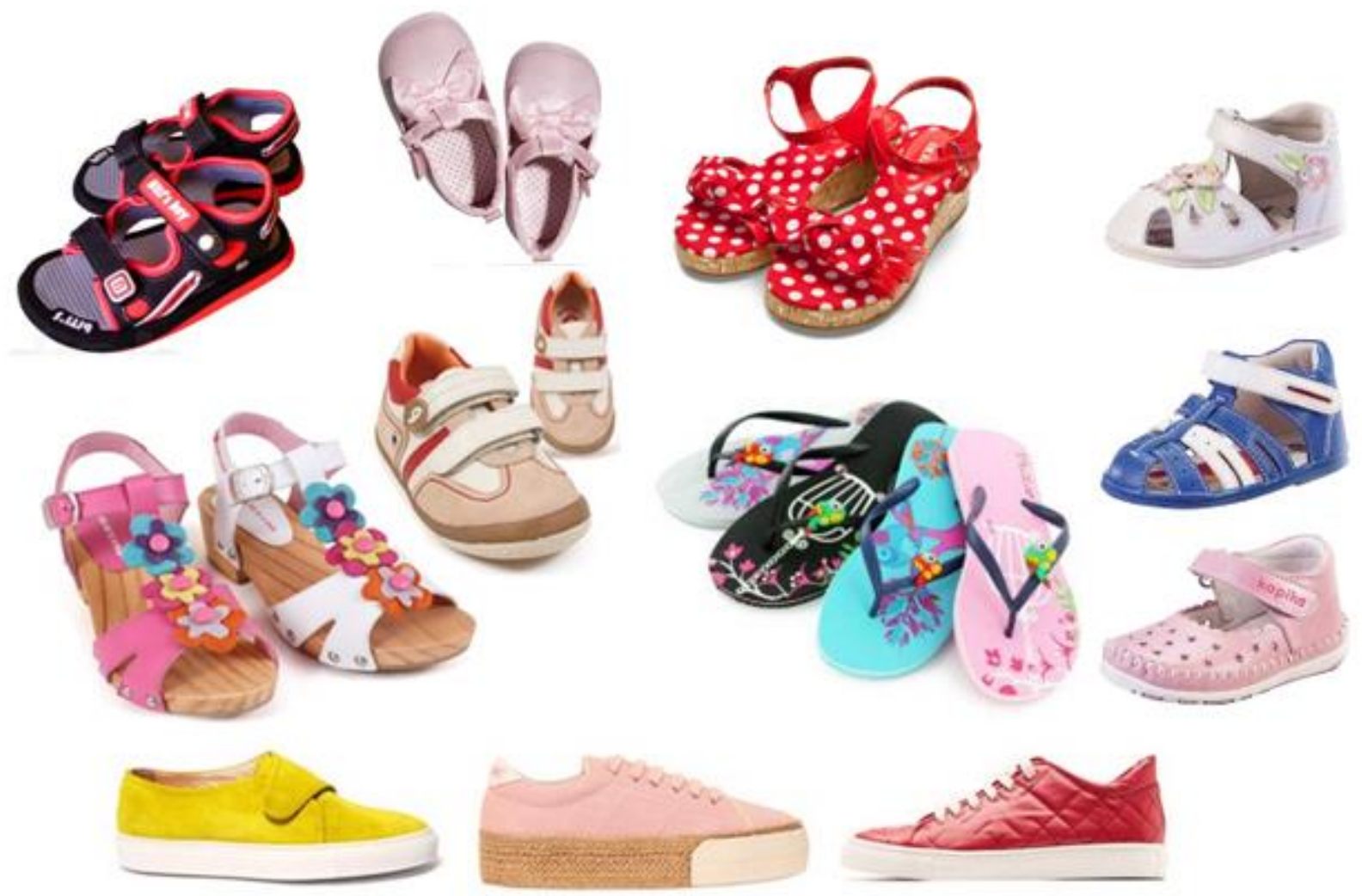

Figure 3 The range of summer children's shoes 


\begin{tabular}{|c|c|c|c|c|c|c|}
\hline \multirow{4}{*}{ Impact Factor: } & ISRA (India) & $=3.117$ & SIS (USA) & $=0.912$ & ICV (Poland) & $=6.630$ \\
\hline & ISI (Dubai, UAE & $=0.829$ & РИНЦ (Russia) & $=0.156$ & PIF (India) & $=1.940$ \\
\hline & GIF (Australia) & $=0.564$ & ESJI (KZ) & $=8.716$ & IBI (India) & $=4.260$ \\
\hline & JIF & $=1.500$ & SJIF (Morocco) & $=5.667$ & OAJI (USA) & $=0.350$ \\
\hline
\end{tabular}

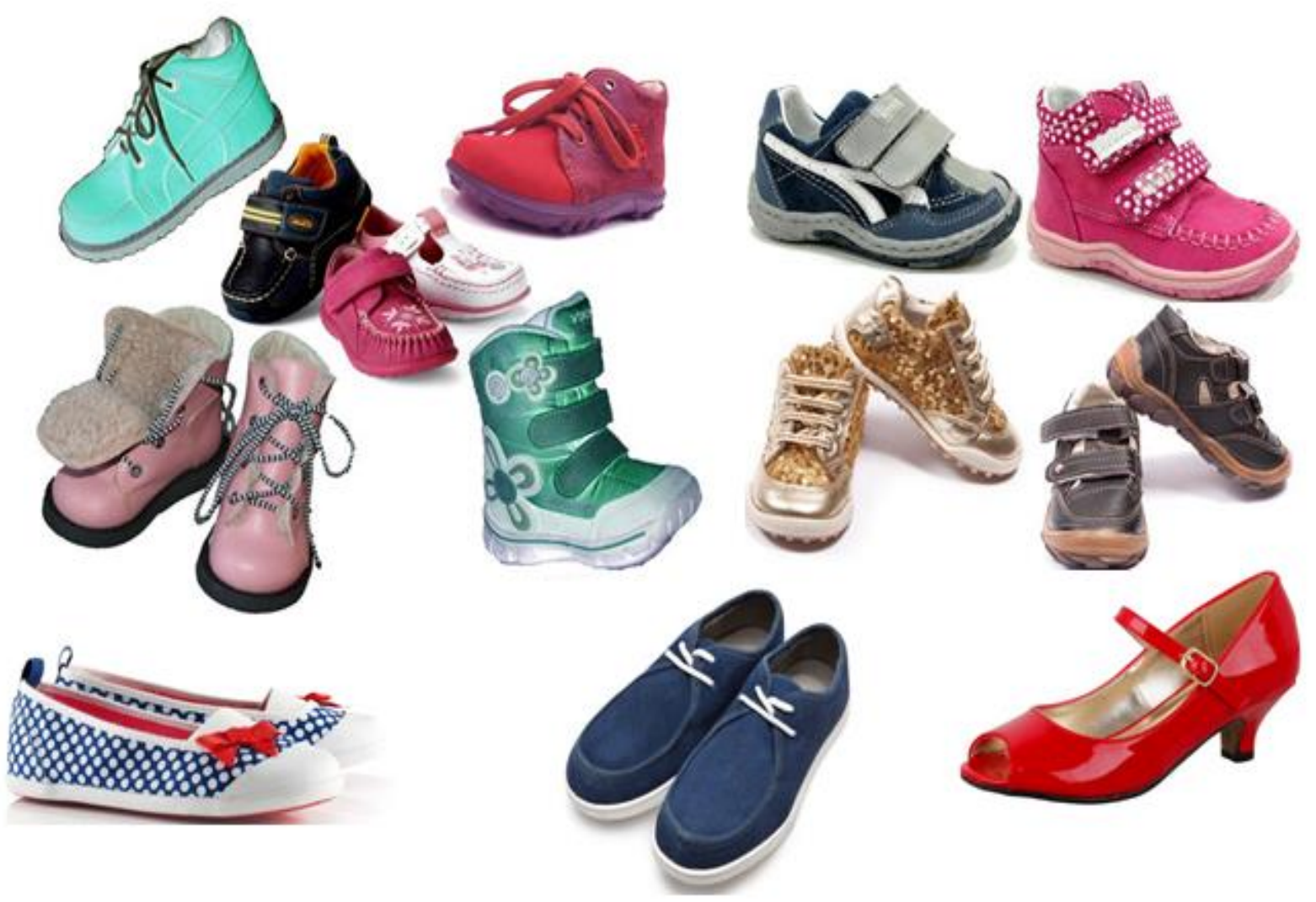

Figure 4 Range of children's shoes for spring

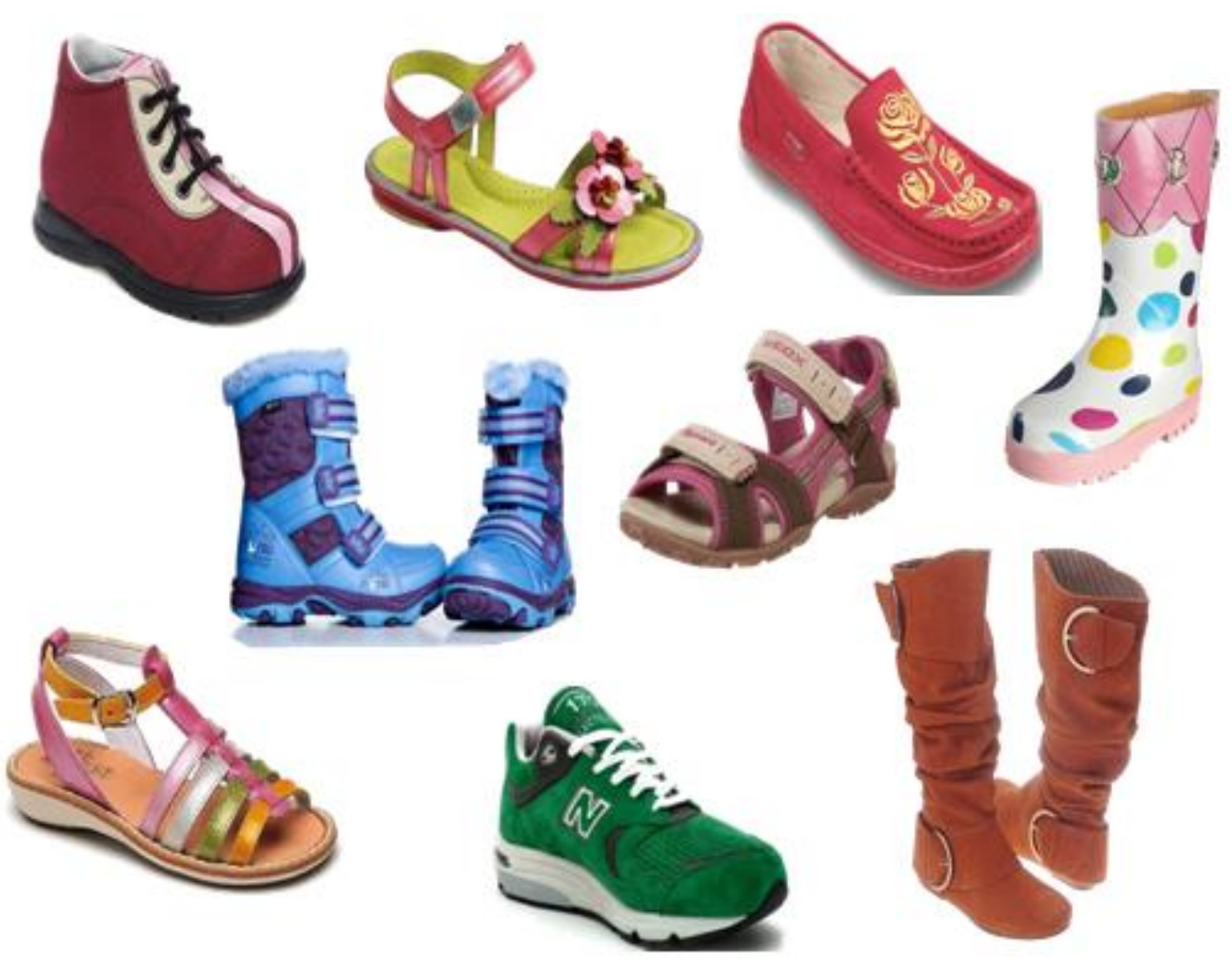

Figure 5 The range of children's shoes for autumn 


\begin{tabular}{llllll} 
& ISRA (India) $=\mathbf{3 . 1 1 7}$ & SIS (USA) & $=\mathbf{0 . 9 1 2}$ & ICV (Poland) & $=\mathbf{6 . 6 3 0}$ \\
Impact Factor: & ISI (Dubai, UAE) $=\mathbf{0 . 8 2 9}$ & PUHL (Russia) $=\mathbf{0 . 1 5 6}$ & PIF (India) & $=\mathbf{1 . 9 4 0}$ \\
& GIF (Australia) $=\mathbf{0 . 5 6 4}$ & ESJI (KZ) $=\mathbf{8 . 7 1 6}$ & IBI (India) & $\mathbf{4 . 2 6 0}$ \\
JIF & $\mathbf{1 . 5 0 0}$ & SJIF (Morocco) $=\mathbf{5 . 6 6 7}$ & OAJI (USA) & $\mathbf{0 . 3 5 0}$ \\
\hline
\end{tabular}

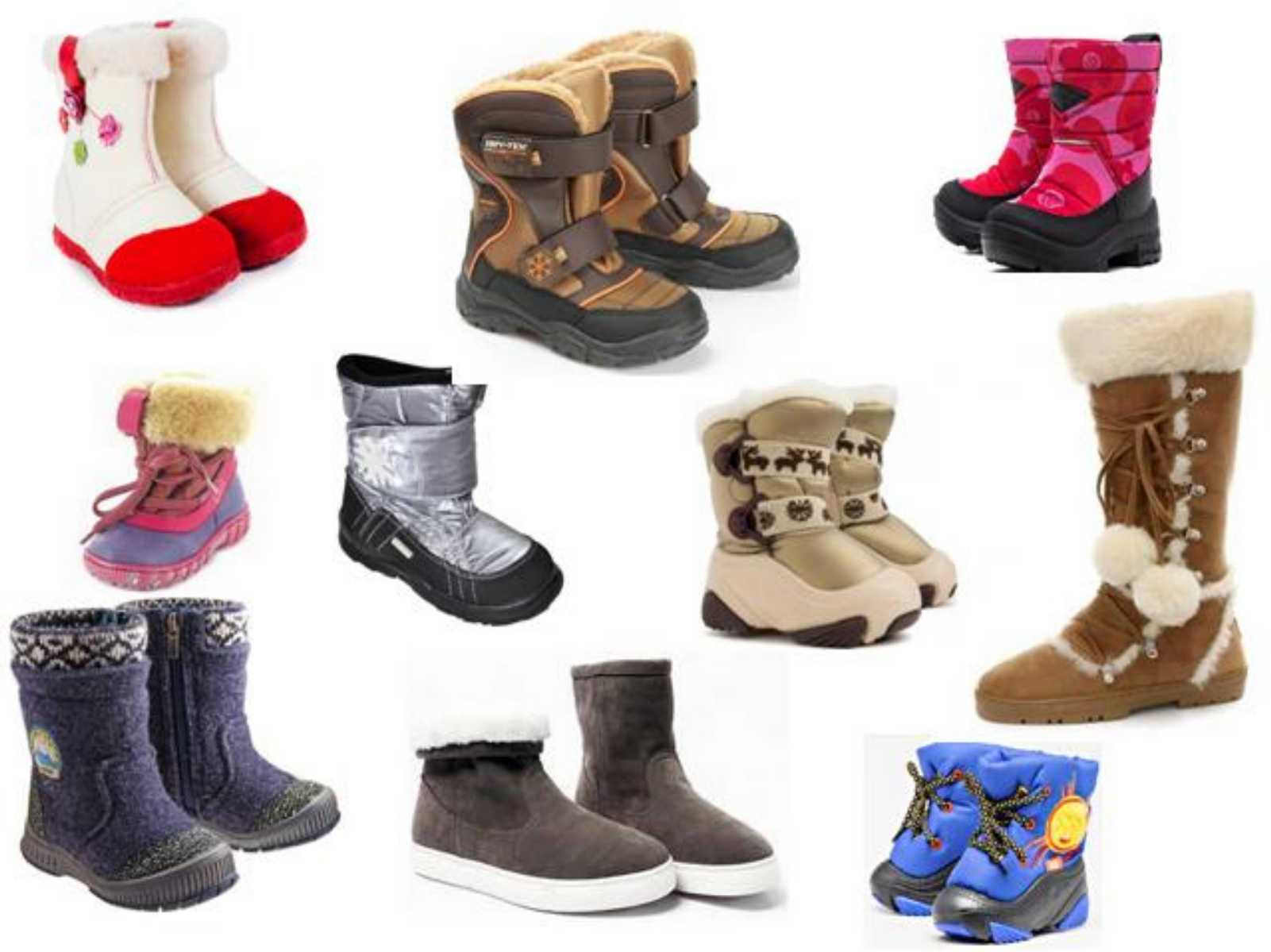

Figure 6 The range of winter children's shoes

Planning and assortment management - an integral part of marketing. Even a well-designed marketing and advertising plans will not be able to neutralize the consequences of mistakes made earlier in the planning range.

The optimum range of the structure should ensure maximum return on the one hand and sufficient stability of the economic and market indicators (eg sales volume), on the other hand. (Ris.1-6)

Achieving the highest possible profitability is ensured by continuous monitoring of economic indicators and timely decisions on the adjustment range.

The coefficient of efficiency of the process is calculated by the formula:

$$
\begin{aligned}
& K_{э \phi}=K_{\Pi т} \cdot K_{3}{ }^{i} \cdot P_{s} \cdot C \cdot S_{\text {обш }} \cdot 3_{\phi} \\
& \cdot T_{\text {б.y. }} \cdot \Pi p \cdot \mathrm{R} \cdot 3_{1 \text { тт. }} \cdot 3_{\text {усл.пер.ед }} \cdot 3_{\text {усл.пос.ед. }} \cdot
\end{aligned}
$$

$$
K_{\Pi T}=\frac{P}{H_{\text {выp }}}
$$

where $\mathrm{P}$ - reference stream pairs;

$H_{\text {выр }}$ - production rate of the project, par.

Loading workers (Kzi)

$$
K_{3}^{i}=\frac{Я c \partial^{P}}{Я c \partial^{\Phi}}
$$

Where $\ell_{c} \partial^{P}$ - the estimated number of workers, people .;

$Я c \partial^{\Phi}$ - the actual number of workers people.

Release shoe on $1 \mathrm{~m} 2$ (Ps)

$$
P_{s}=\frac{P}{S_{n p}}
$$

Labor productivity (CBT) 


\begin{tabular}{|c|c|c|c|c|c|c|}
\hline \multirow{4}{*}{ Impact Factor: } & ISRA (India) & $=3.117$ & SIS (USA) & $=0.912$ & ICV (Poland) & $=6.630$ \\
\hline & ISI (Dubai, UAE & $=0.829$ & РИНЦ (Russia) & $=0.156$ & PIF (India) & $=1.940$ \\
\hline & GIF (Australia) & $=0.564$ & ESJI (KZ) & $=8.716$ & IBI (India) & $=4.260$ \\
\hline & JIF & $=1.500$ & SJIF (Morocco) & $=5.667$ & OAJI (USA) & $=0.350$ \\
\hline
\end{tabular}

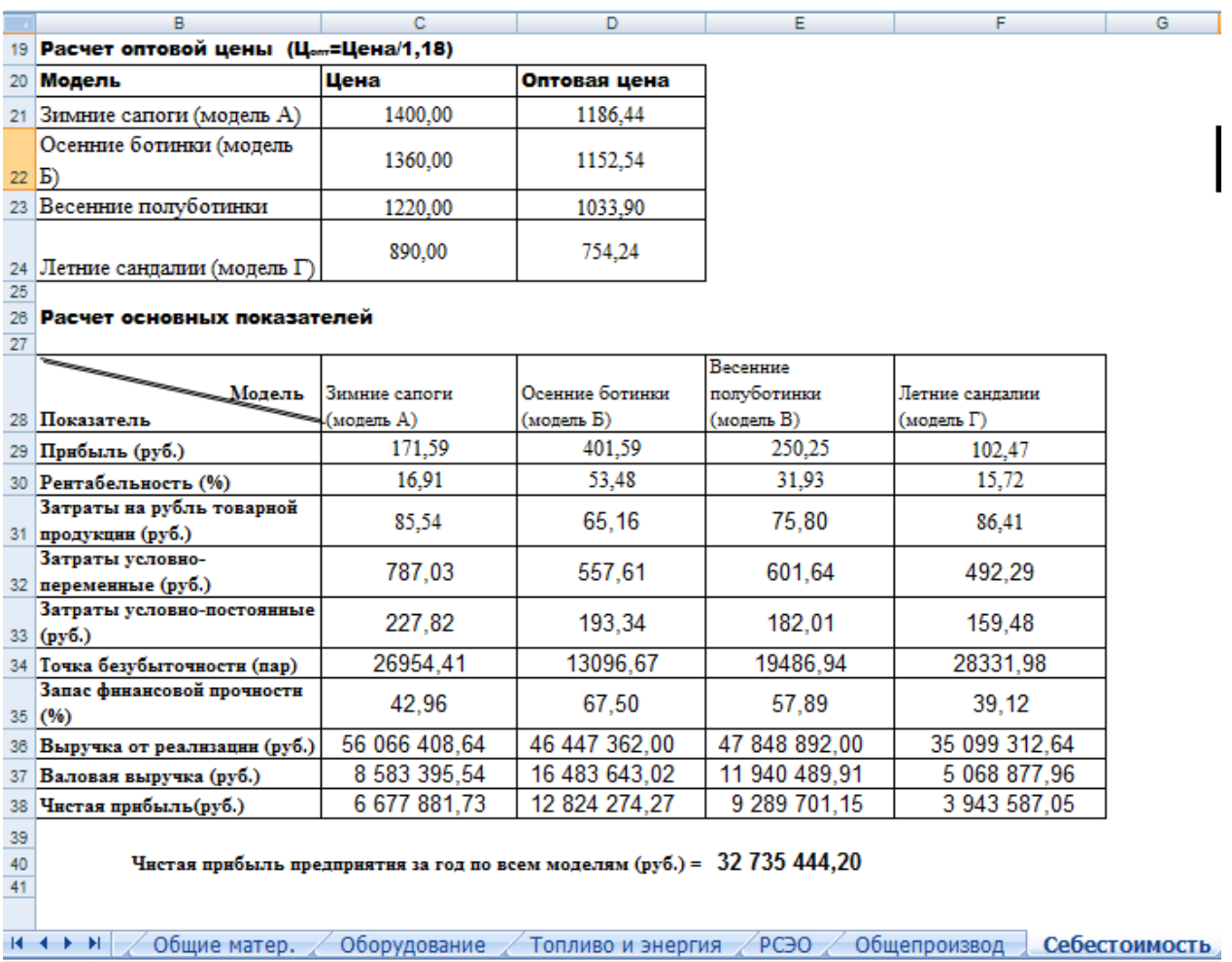

Fig. 7 - Calculation of basic economic indicators ( "Cost" sheet).

\begin{tabular}{|c|c|c|c|c|c|}
\hline \multirow{2}{*}{\multicolumn{6}{|c|}{$\begin{array}{l}4 \\
\text { te, об́естечнивающеe выmvск всет модетей }\end{array}$}} \\
\hline & & & & & \\
\hline $\begin{array}{l}\text { Напьенование } \\
\text { оборудования }\end{array}$ & $\begin{array}{c}\text { Количество } \\
\text { оборудования, шт. }\end{array}$ & $\begin{array}{c}\text { Мощность } \\
\text { злектродвигателт, } \mathrm{kB} \text {. }\end{array}$ & $\begin{array}{c}\text { Установленна } \\
\text { я момшность, } \\
\text { вB }\end{array}$ & $\begin{array}{c}\text { Цена за единицу } \\
\text { о6орудования, ру6. }\end{array}$ & $\begin{array}{c}\text { Слоивость } \\
\text { о6орудования, ру6. }\end{array}$ \\
\hline $3 \mathrm{S120 \textrm {C }}$ & 9 & 1,1 & 9,9 & 27300 & 245700 \\
\hline 4 HSP588/3 & 2 & 0,8 & 1,6 & 54000 & 108000 \\
\hline$5 \longdiv { \text { SS 20 } }$ & 3 & 0,5 & 1,5 & 15900 & 47700 \\
\hline 6 A.2000 & 2 & 2,1 & 4,2 & 127000 & 254000 \\
\hline 7 RP67TE & 3 & 1 & 3 & 37800 & 113400 \\
\hline 8 Швейные & 4 & 0,27 & 1,08 & 17560 & 70240 \\
\hline 9 Pfaff 574-900 & 4 & 0,27 & 1,08 & 79600 & 318400 \\
\hline 10 Pfaff $1243-750,01$ & 1 & 0,27 & 0,27 & 79400 & 79400 \\
\hline $11 \mathrm{GP} 2$ & 1 & 0,27 & 0,27 & 19000 & 19000 \\
\hline 12 GRAMAC 652 & 2 & 0,27 & 0,54 & 21300 & 42600 \\
\hline $1302015 / \mathrm{P} 5$ & 1 & 0,23 & 0,23 & 42600 & 42600 \\
\hline$1 4 \longdiv { 1 0 / 1 1 / C }$ & 2 & 0,5 & 1 & 51300 & 102600 \\
\hline 1200 & 1 & 0,25 & 0,25 & 54000 & 54000 \\
\hline $16 \mathrm{CD} 3000 \mathrm{U}$ & 2 & 2,7 & 5,4 & 35700 & 71400 \\
\hline 17 Терысоактие. 133 & 1 & 4,3 & 4,3 & 130000 & 130000 \\
\hline 18 AS $1880 \mathrm{~K}$ & 1 & 7 & 7 & 252600 & 252600 \\
\hline $19 \overline{\mathrm{FO} \mathrm{2016}}$ & 1 & 3 & 3 & 87000 & 87000 \\
\hline $20 \mathrm{G} 504 \mathrm{CF}$ & 1 & 1,2 & 1,2 & 15700 & 15700 \\
\hline 21 SR 1006 & 2 & 0,18 & 0,36 & 29000 & 58000 \\
\hline $22 \mathrm{G} 12 / 1$ & 2 & 1,9 & 3,8 & 54000 & 108000 \\
\hline 23 K73STIC & 1 & 5,5 & 5,5 & 157680 & 157680 \\
\hline 24 PIC K24SZ & 1 & 5,5 & 5,5 & 285100 & 285100 \\
\hline$2 5 \longdiv { 0 2 0 6 8 / \mathrm { P } 4 }$ & 2 & 0,6 & 1,2 & 11200 & 22400 \\
\hline $2501276 / \mathrm{P} 12$ & 2 & 0,18 & 0,36 & 18000 & 36000 \\
\hline$2 7 \longdiv { \mathrm { TL } 7 5 }$ & 1 & 0,1 & 0,1 & 15200 & 15200 \\
\hline $2804222 \mathrm{Pl}$ & 1 & 0,42 & 0,42 & 49400 & 49400 \\
\hline $2905054 / \mathrm{P} 1$ & 1 & 0,25 & 0,25 & 12300 & 12300 \\
\hline 30 FR 3500 & 1 & 13 & 13 & 41200 & 41200 \\
\hline Конвейер & 1 & 1,1 & 1,1 & 125000 & 125000 \\
\hline \multicolumn{6}{|l|}{32} \\
\hline 33 & & & & & 0 \\
\hline 34 И7oro & 56 & & $\begin{array}{lll}77.41 \\
\end{array}$ & & 2964620 \\
\hline \multicolumn{2}{|c|}{ 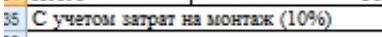 } & & & & 3261082 \\
\hline
\end{tabular}

Fig. 8 - Calculation of the costs for maintenance and operation of equipment ( "Equipment" page). 


\begin{tabular}{llllll} 
& ISRA (India) $=\mathbf{3 . 1 1 7}$ & SIS (USA) & $=\mathbf{0 . 9 1 2}$ & ICV (Poland) & $=\mathbf{6 . 6 3 0}$ \\
Impact Factor: & ISI (Dubai, UAE) $=\mathbf{0 . 8 2 9}$ & PUHL (Russia) $=\mathbf{0 . 1 5 6}$ & PIF (India) & $=\mathbf{1 . 9 4 0}$ \\
& GIF (Australia) $=\mathbf{0 . 5 6 4}$ & ESJI (KZ) $=\mathbf{8 . 7 1 6}$ & IBI (India) & $=\mathbf{4 . 2 6 0}$ \\
& JIF & $\mathbf{1 . 5 0 0}$ & SJIF (Morocco) $=\mathbf{5 . 6 6 7}$ & OAJI (USA) & $\mathbf{0 . 3 5 0}$ \\
\hline
\end{tabular}

\begin{tabular}{|c|c|c|c|c|c|c|c|c|}
\hline & 1 & 2 & 3 & 4 & 5 & 6 & 7 & 8 \\
\hline 1. & \multicolumn{8}{|c|}{ Пропзводственная программа на год в натуратьном выраженен } \\
\hline 2 & \multirow[b]{2}{*}{ Наронование ияпелий } & \multirow{2}{*}{$\begin{array}{c}\text { Выпуск изделий } \\
\text { в день, пар }\end{array}$} & \multirow{2}{*}{\begin{tabular}{|c|}
$\begin{array}{c}\text { Период выпуска } \\
\text { изпелия в течения } \\
\text { года, дня }\end{array}$ \\
\end{tabular}} & \multirow{2}{*}{$\begin{array}{c}\text { Buпуск изделий sa } \\
\text { год, пар }\end{array}$} & \multicolumn{4}{|c|}{ В томь числе по кварталам } \\
\hline 3 & & & & & I & II & III & IV \\
\hline 4 & 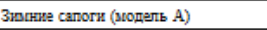 & 716 & 66 & 47256 & & & 47256 & \\
\hline 5 & Oсенние ботинки (Модель Б) & 650 & 62 & 40300 & & 40300 & & \\
\hline 6 & Весенние полуботинки (модель В) & 712 & 65 & 46280 & & & & 46280 \\
\hline 7 & Летние сандалия (модель Г) & 831 & 56 & 46536 & 46536 & & & \\
\hline 8 & Итоге: & & 249 & 180372 & 46536 & 40300 & 47256 & 46280 \\
\hline $\begin{array}{c}9 \\
10\end{array}$ & \multicolumn{8}{|c|}{ Пропзводственная программа на год в стогмостном выраженни } \\
\hline 11 & \multirow{2}{*}{ Hаньенование изпелий } & \multirow{2}{*}{\begin{tabular}{|c|} 
Годовой выпуск \\
изпелия, пар
\end{tabular}} & \multirow{2}{*}{$\begin{array}{c}\text { Стоиьость } \\
\text { изделия, ру6. }\end{array}$} & \multirow{2}{*}{$\begin{array}{c}\text { Годовой объем! } \\
\text { выпуска, тыс.ру6. } \\
\end{array}$} & \multicolumn{4}{|c|}{ В том числе no kвspталам } \\
\hline 12 & & & & & I & II & III & IV \\
\hline 13 & 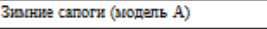 & 47256 & 1400 & 66158,4 & & & 66158,4 & \\
\hline 14 & Oсенние 6отинки (модель Б) & 40300 & 1360 & 54808 & & 54808 & & \\
\hline 15 & Весенние полуботинки (модель В) & 46280 & 1220 & 56461,6 & & & & 56461,6 \\
\hline 16 & Летние сандалик (морель $\Gamma$ ) & 46536 & 890 & 41417,04 & 41417 & & & \\
\hline 17 & Iтroro: & & & 218845,04 & 41417 & 54808 & 66158,4 & 56461,6 \\
\hline 18 & & взводственная 마 & porрамма в трудо- & qacax & & & & \\
\hline 19 & \multirow{2}{*}{ Найенование изделий } & \multirow{2}{*}{$\begin{array}{c}\text { Годовой выпуск } \\
\text { взпелия, пар }\end{array}$} & \multirow{2}{*}{$\begin{array}{c}\text { Tрудоегквость } \\
\text { нвделия }\end{array}$} & \multirow{2}{*}{\begin{tabular}{|c|} 
Годовой обвеэt \\
выпуска, в трудо- \\
vacev
\end{tabular}} & \multicolumn{4}{|c|}{ В тos: числе no кEapranaus } \\
\hline 20. & & & & & I & II & III & IV \\
\hline 21 & Зияние сапоги (модель A) & 47256 & 0,66 & 31188,960 & & & 31189 & \\
\hline 22 & Осенние болинки (модепь Б) & 40300 & 0,73 & 29419,000 & & 29419 & & \\
\hline 23 & Весенние полуболинки (модель В) & 46280 & 0,582 & 26934,960 & & & & 26934,96 \\
\hline 24 & Леткиа сандалия (модель $\Gamma$ ) & 46536 & 0,56 & 26060,160 & 26060,2 & & & \\
\hline 25 & Иrore: & & & 113603,08 & 26060,2 & 29419 & 31189 & 26934,96 \\
\hline 28 & & & & & & & & \\
\hline 27 & & & & & & & & \\
\hline 28 & & & & & & & & \\
\hline 29 & & & & & & & & \\
\hline 30 & & & & & & & & \\
\hline 31 & & & & & & & & \\
\hline
\end{tabular}

Fig. 9 - Calculation of the production program of the enterprise for the year (Sheet "'" Proizvod.progr. ").

Where $S_{n p}$ - manufacturing area, m2.

The cost of equipment per unit of task flow (C)

$$
C=\frac{T}{P},
$$

where $\mathrm{T}$ - the cost of equipment, rubles.

The total rate (Sobsch)

$$
S_{\text {общ }}=\sum_{i=1}^{n} S^{i}
$$

Where $S^{i}$ - rate at the i-th step;

$\mathrm{n}$ - number of operations.

Financial strength calculated by the following equation (FPC)

$$
\mathrm{FPC}=\frac{B_{2}-T_{6 . y}}{B_{2}} \cdot 100(\%)
$$

where B2 - commodity output in the planning period in real terms, the pair;

Tb.u - break-even point pair. (Tb.u):

break-even point is determined by the formula

$$
\mathrm{Tb} . u=\frac{3_{\text {усл.пост. }}}{Ц_{\text {ед. }}-3_{\text {усл.пер.ед }} .} \text { (Pairs), }
$$

where Zusl.post - total fixed costs of manufacturing production units, rub .;

Tsed.- unit price, rbl .;

Zusl.per.ed. - total variable costs of production units of production, rbl.

Profit per unit of output (approx.) Is determined by the following relationship:

$$
\text { Etc. Tsopt }=-\mathrm{C}
$$

where Tsopt - wholesale unit price (selling price minus VAT of $10 \%$ for children's shoes and $18 \%$ for other types), rub;

$\mathrm{C}$ - complete unit cost, rubles.

The profitability of the product $(\mathrm{R})$ is determined by the following formula:

$$
\mathrm{R}=\frac{\Pi_{p}}{C} \cdot 100(\%),
$$

where $\operatorname{Pr}$ - the profit from the sale of a unit of production, rub ;

C - complete unit cost, rubles.

The cost of 1 rub. marketable products (Z1r etc.) is determined by the following formula:

$$
\begin{aligned}
& \text { Z1r etc. }=\frac{C}{\bigsqcup_{\text {onm }}} \cdot 100 \quad(\text { Cop }),(11) \\
& \text { where } \mathrm{C}-\text { full unit cost of production, rbl. }
\end{aligned}
$$




\begin{tabular}{|c|c|c|c|c|c|c|}
\hline \multirow{4}{*}{ Impact Factor: } & ISRA (India) & $=3.117$ & SIS (USA) & $=0.912$ & ICV (Poland) & $=6.630$ \\
\hline & ISI (Dubai, UAE & $=0.829$ & РИНЦ (Russia & $=0.156$ & PIF (India) & $=1.940$ \\
\hline & GIF (Australia) & $=0.564$ & ESJI (KZ) & $=8.716$ & IBI (India) & $=4.260$ \\
\hline & JIF & $=1.500$ & SJIF (Morocce & $=5.667$ & OAJI (USA) & $=0.350$ \\
\hline
\end{tabular}

Tsopt - wholesale unit price (selling price less the value added tax of $10 \%$ for children's shoes and $18 \%$ for other types), rub.

Semi-variable costs (total variable cost of a unit of production) (. Zusl per.ed.) is defined as:

Zusl. before. Spol $=-(.5$ st.s.pol st.s.pol $+6+7$ +8 st.s.pol st.s.pol st.s.pol $+9 \ldots .$. ; (Rub.) (12)

Fixed costs (total fixed costs per unit of output production) (Zusl. Pos.ed.)

Zusl. pos.ed $=$ spol. $-(.1$ st.s.pol st.s.pol $+2+3$ + 4 st.s.pol st.s.pol...); (Rub.) (13)

And software for optimal power has been developed

At the same time as the criteria for informed choice of an optimum power in the formation of the algorithm is justified were chosen precisely those criteria that have the greatest impact on the cost of the finished product, namely:

- loss of wages per unit of output, rub .;

- release of shoe $1 \mathrm{~m} 2$;

- the percentage of the working load, $\%$;

- productivity per worker pair; rub ;

- given the specific costs of 100 pairs of shoes,

- the cost of equipment per unit of task flow (C)

- total rate (Sobsch);

- financial strength (FPC);

- break-even point (Tb.u);

- the profit per unit of output (Prov.);

- profitability of products (R);

- the cost of 1 rub. commodity products (Z1r etc.);

- semi-variable costs (Zusl per.ed..);

- Fixed costs (Zusl pos.ed.).

From these criteria, in our opinion, the manufacturer is able to give preference to those from his point of view, it would guarantee the production of import-substituting, competitive and marketable products, namely:

- Labor 1 worker productivity - a key indicator of labor. The level and dynamics of labor productivity depend to a greater or lesser extent all the key performance indicators of production and all the labor indicators:.. Production, number of employees, the expenditure of wages, wages, etc., to improve productivity are paramount introduction of new engineering and technology, extensive mechanization of labor-intensive work, the automation of production processes, training of staff and workers, especially in the implementation of innovative techno ogicheskih processes on the basis of universal and multifunctional equipment;

- given the specific costs - an indicator of comparative economic efficiency of capital investments used in selecting the best of the solutions to technological problems ;
- reduced costs - the amount of current costs accounted in the cost of production and non-recurring capital investments, comparability with current costs which is achieved by multiplying them by a regulatory factor capital efficiency;

- financial strength (ZFP) shows how many percent of the company can reduce the sales volume without incurring losses;

- break-even point allows (Tb.u) to determine the minimum required volume of product sales, in which the company covers its costs and break even works without giving profit, but also does not suffer losses, that is a minimum output size that income equality is achieved from sales and costs of production;

- Profit (loss) from sale of goods (PR) is defined as the difference between revenue from sales in current prices, VAT and excise taxes and the cost of its production and sales;

- the profitability of the product $(\mathrm{R})$ represents the relationship between the profit from the sale of units of production and its cost;

- fixed costs (total fixed costs per unit of output production) (Zusl.pos.ed) that vary in proportion or almost proportion to the volume of production (1st Class - cost of raw materials and materials; 2 st - costs of auxiliary materials; $3 \mathrm{st}$ - costs fuel and energy for technological needs; 4st - costs and additional basic wage of production workers with insurance premiums to non-budgetary funds);

- semi-variable costs (total variable cost of a unit of production) (Zusl per.ed..), Which are independent or nearly independent of changes in the volume of production (5CT - costs for the preparation and development costs of production; Article 6 - costs the cost of maintaining and operating the equipment; 7st the cost of overhead needs; 8st - costs for general expenses, they, along with fixed costs make up the production cost; Article 9 - on business expenses costs all of these articles. and - forming and semi-variable costs and fixed costs make up the full cost, ie semivariable costs are defined as the total cost - fixed costs, and vice versa,

- the cost of 1 rub. commodity production show the relative size of profit on each ruble of current expenditure, ie the ratio of unit cost to the wholesale price, which characterizes the efficiency of carried out measures to improve the competitiveness of and demand for the product on the market demand.

To translate dimension indicators assessments are encouraged to use the dimensionless index method. Indices dimensionless parameters determined by the formula (18) for positive performance, with a positive trend - the growth (eg, return on sales, productivity) and the formula (19) to the negative performance, with a positive trend - decrease (eg, depreciation, excess inventory of finished products in stock compared with the norm, the turnover rate), taken mainly of the indicators that form the cost of production: 


\begin{tabular}{llllll} 
& ISRA (India) $=\mathbf{3 . 1 1 7}$ & SIS (USA) & $=\mathbf{0 . 9 1 2}$ & ICV (Poland) & $=\mathbf{6 . 6 3 0}$ \\
Impact Factor: & ISI (Dubai, UAE) $=\mathbf{0 . 8 2 9}$ & PUHL (Russia) $=\mathbf{0 . 1 5 6}$ & PIF (India) & $=\mathbf{1 . 9 4 0}$ \\
& GIF (Australia) $=\mathbf{0 . 5 6 4}$ & ESJI (KZ) & $=\mathbf{8 . 7 1 6}$ & IBI (India) & $=\mathbf{4 . 2 6 0}$ \\
& JIF & $\mathbf{1 . 5 0 0}$ & SJIF (Morocco) $=\mathbf{5 . 6 6 7}$ & OAJI (USA) & $\mathbf{0 . 3 5 0}$ \\
\hline
\end{tabular}

$$
\begin{aligned}
& O_{i}=X_{i} / X_{i}^{\max }, \\
& O_{i}=X_{i}^{\min } / X_{i},
\end{aligned}
$$

where Oi - dimensionless (index) score of i-index of competitiveness of the enterprise,

$x i$ - the value of the i-th dimension index evaluation of enterprise competitiveness,

Himax - the maximum value of i-dimensional index evaluation of enterprise competitiveness,

Himin- the minimum value of i-dimensional index evaluation of enterprise competitiveness.

Step 4: Estimation of competitiveness of the goods. It carried out for light industry products in their domestic demand.

Step 5. Calculation of summary measure the competitiveness of enterprises. Quantitation of competitiveness of the enterprise is proposed to determine the following formula (3).

$$
K_{\Pi}=\sum_{i=1}^{m} \alpha_{i} \times O_{i}
$$

where KP - an estimation of competitiveness of the enterprise as a percentage, $\alpha_{i}$ - the importance of the i-th index of competitiveness as a percentage,

$O i$ - index (dimensionless) evaluation of i-index of competitiveness,

$m$ - the number of indicators of competitiveness of the enterprise valuation.

Evaluation value competitiveness of the enterprise can theoretically range from 0 to 100 (ratio of 21).

$$
K n=0 \div 100
$$

For qualitative characteristics of the obtained competitive assessment scale required quality level evaluation. In economic practice, a principle of the scales at regular intervals, progressive and regressive scale. Progressive and regressive scale is most often used for material incentives. We believe that is the most appropriate scale with equal pitch, because it firstly corresponds to the solution of practical problems (qualitative specification competitiveness), secondly, the construction and easy to use. Step scale is defined as a 100 (maximum score): 4 (number of levels) $=25$. You can select and other step values that defined goals and objectives, which currently generates the enterprise itself.

Table 9. The scale of assessment of quality of the enterprise competitiveness

\begin{tabular}{|l|l|}
\hline Evaluation Percentage & quality of \\
\hline from 0 to 24.9 & very low \\
\hline from 25.0 to 49.9 & low \\
\hline from 50.0 to 74.9 & average \\
\hline from 75.0 to 100 & tall \\
\hline
\end{tabular}

$\mathrm{Keff}=\mathrm{K} 1 \cdot \mathrm{K} 2 \cdot \mathrm{K} 3 \cdot \mathrm{K} 4 \cdot \mathrm{K} 5 \cdot \mathrm{K} 6 \cdot \mathrm{K} 7 \cdot \mathrm{K} 8 \cdot \mathrm{K} 9 \mathrm{~K} 10 \cdot \mathrm{K} 11 \cdot \mathrm{K} 12,(18)$

wherein Keff - weighting coefficient of efficiency evaluation of technological processes for the production of formed competitive and marketable products

$\mathrm{K} 1$ - the weight of labor productivity (PT);

$\mathrm{K} 2$ - the weight of the working load (WP);

$\mathrm{K} 3$ - the weight of the shoe release (Ps);

$\mathrm{K} 4$ - the weight of equipment cost per unit of the job flow (C);

K5 - the weight of the total rates per unit of output (Sobsch); (FPC);

K6 - the weight of a stock of financial strength

K7 - the weight of the break-even point (Tb.u);

$\mathrm{K} 8$ - the weight of a unit of production profits (Prov.);

K9 - weight of profitability of products (R);
K10 - the weight of the cost of 1 ruble of marketable products (Z1r.t.p);

K11 - the weight of semi-variable costs (total variable cost of a unit of production) (Zusl.per.ed);

K12 - the weight of fixed costs (total fixed costs per unit of output production) (Zusl.pos.ed)

Cost of services and products - is the current expenses of the enterprise for production and sale of products and services, expressed in monetary terms. When calculating the cost of products and services all company expenses are classified according to various criteria:

- depending on the nature of their assignment in the cost of services and products are divided into 2 groups: direct and indirect.

direct are those costs that can be directly attributed to a particular type of product in the 


\begin{tabular}{|c|c|c|c|c|c|c|}
\hline \multirow{4}{*}{ Impact Factor: } & ISRA (India) & $=3.117$ & SIS (USA) & $=0.912$ & ICV (Poland) & $=6.630$ \\
\hline & ISI (Dubai, UAE & $=0.829$ & РИНЦ (Russia & $=0.156$ & PIF (India) & $=1.940$ \\
\hline & GIF (Australia) & $=0.564$ & ESJI (KZ) & $=8.716$ & IBI (India) & $=4.260$ \\
\hline & JIF & $=1.500$ & SJIF (Morocce & $=5.667$ & OAJI (USA) & $=0.350$ \\
\hline
\end{tabular}

formulation of more than one of its kind (material, fuel, energy).

indirect - costs that can not be directly attributed to the cost of various products in the manufacture and repair of more than one of its kind, and then distributed among them in proportion to other costs or labor resources.

- depending on changes in the volume of production all costs are divided into semi-variable (proportional) and conventionally fixed (nonproportional).

By conditional variables are costs that change in proportion or almost proportion to the volume of production (cost of materials and energy for technological purposes, wages of production workers, etc.).

Conditionally-constant are costs that are independent or nearly independent of changes in the volume of production (depreciation of the value of fixed assets, leasing and maintenance costs of buildings and facilities, salaries of managers, professionals and employees, etc.):

- on the economic role in the production process: basic and overhead; complex;

- composition (homogeneity): single-element,

- the frequency of occurrence of: current and non-recurring.

One-time - the costs of training and development of production new products, the costs associated with the launch of new businesses and more:

- to participate in the production process: production and sales;

- Performance: productive, unproductive.

Productive costs considered on production of the established quality at a rational technology and organization of production.

unproductive spending They are the result of deficiencies in the organization of production technology (loss of downtime, marriage product overtime et al.).

Planned manufacturing costs and overhead are not planned.

Calculation of the cost of services and products It referred to the definition of the cost of manufactured products and services, carried out by cost category. Calculate the cost if calculation is made on the standard units accrued.

Cost estimate compiled according to the nomenclature of calculation Articles:

1. Raw materials and basic materials (taking into account the transportation and procurement costs and net realizable waste).

2. Auxiliary materials.

3. Fuel and electricity for technological purposes.
4. The basic and additional wages of production workers insurance contributions to the extrabudgetary funds.

5. Expenditure on training and development of production.

6. Expenditures for maintenance and operation of the equipment (RSEO)

7. Overhead costs (shop costs).

8. General running costs.

9. Payments for compulsory property insurance.

actual manufacturing cost

10. Commercial (Non-manufacturing) costs.

The total cost.

Estimated costs of production and financial results

To determine the total amount of the planned costs in the company and alignment indicators cost, profit and profitability with the other figure is an estimate of the cost of production on economic elements, which include the cost of all structural divisions of the enterprise involved in the performance of services (manufacturing output).

cost estimate considered a consolidated document outlining the monetary value of all the material and energy costs required to ensure the implementation of the plan for the production of goods and services.

The costs accounted in the estimates, grouped as follows.

Costings

1. Raw materials and basic materials.

2. Auxiliary materials.

3. Purchased products and semifinished products.

4. Fuel from the side.

5. The energy from the outside.

6. The basic and additional wages of industrial personnel (PPP) with deductions on the unified social tax.

7. Depreciation of fixed assets for a full recovery.

8. Other expenses.

Formation of financial results. The final financial result (profit or loss) is composed of the financial result from the sale of goods (works, services), fixed assets and other assets of the company and non-operating income, reduced by the amount of expenses related to these operations.

Profit Loss) from the sale of goods (works, services) and the goods shall be determined as the difference between the proceeds from the sale of goods (works, services) in current prices excluding VAT and excise duties and the cost of its production and sales. 


\begin{tabular}{|c|c|c|c|c|c|c|}
\hline \multirow{4}{*}{ Impact Factor: } & ISRA (India) & $=3.117$ & SIS (USA) & $=0.912$ & ICV (Poland) & $=6.630$ \\
\hline & ISI (Dubai, UAE & $=0.829$ & РИНЦ (Russia & $=0.156$ & PIF (India) & $=1.940$ \\
\hline & GIF (Australia) & $=0.564$ & ESJI (KZ) & $=8.716$ & IBI (India) & $=4.260$ \\
\hline & JIF & $=1.500$ & SJIF (Morocce & $=5.667$ & OAJI (USA) & $=0.350$ \\
\hline
\end{tabular}

Planned profit $(P P L)$ :

$\Pi_{\text {пл }}=(\mathrm{B} \cdot Ц)-(B \cdot \mathrm{C})$, (nineteen $)$ real terms:

$\mathrm{B}$ - commodity output in the planning period in

$\mathrm{P}$ - price for 1 pair of shoes (unit) exclusive of VAT and excise taxes - it is the wholesale price.

$\mathrm{C}$ - the cost of a complete production unit.

Gains one pair (Pl):

$$
\Pi_{1}=U_{\text {on }}-C^{1} \text {, }
$$

where Tsopt - the price of wholesale 1 pair,

$\mathrm{C} 1$ - cost of one pair.

Product profitability reflects the relationship between the profit from the sale of products and their cost.

It shows the relative size of profit Mr every ruble of current expenses and is determined by the formula:

$$
R_{n}=\frac{\Pi_{p}}{3} \cdot 100,
$$

where - the profitability of production; $R_{n}$

Etc. - income from the sale of products;

$\mathrm{W}$ - costs (cost price).

$$
R=\frac{\Pi}{C / C} \cdot 100(\%) \text {-calculation } 1 \text { pair. }
$$

Revenues from sales of products (Works and services) is determined either as of its payment, or at least the shipment of goods (works, services) and presentation of the buyer (customer) payment documents.

incomes relate:

- income received in the territory of the Russian Federation and abroad from equity participation in other companies, dividends and income from bonds and other securities belonging to the enterprise;

- $\quad$ income from the lease of property;

- income from revaluation surplus inventory, and finished goods;

- awarded or recognized by debtors fines, penalties and other sanctions for breach of commercial contracts, as well as income from the compensation of losses;

- earnings from previous years revealed in the reporting year;

- Other income from operations directly related to the production and sale of goods (works and services).

Expenses and losses relate:

- maintenance costs and production capacity conserved objects (other than cost of recoverable from other sources);
- not offset by the perpetrators of the losses from downtime due to external causes;

- losses from the write-down of inventories and finished products;

- loss from packaging;

- legal fees and arbitration costs;

- $\quad$ awarded or recognized fines, penalties and other sanctions for breach of commercial contracts, as well as costs for compensation of damages;

- losses of previous years, identified in the current year;

- not offset by losses due to fires, accidents and other emergency situations caused by extreme conditions; not offset by losses from natural disasters (destruction or damage of inventories of finished goods and other tangible assets, losses from suspension of production, etc.), including the costs associated with the liquidation of consequences of natural disasters; losses from theft, the perpetrators have not been established by the court.

Break-even analysis to determine the minimum required volume of sales, in which the company covers its costs and break even works without giving profit, but also does not suffer losses.

In the most general form of activity of any enterprise carried on a "cost - the production process the profit."

The breakeven point ( $\mathrm{Tb} / \mathrm{y})$ is determined by calculation by the following formula

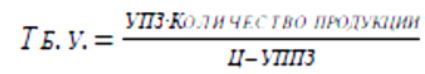

where CPL - conditionally fixed costs per unit of product, rub .; FÁS - semi-variable costs per unit of product, rub .; $\mathrm{C}$ - the unit price exclusive of VAT, rbl.

To plot the break-even should make the following equation:

$$
\begin{aligned}
& y 1=a x ; \\
& y 2=a o+a x
\end{aligned}
$$

where U1 - Revenue, RUR; v2 - the cost (total cost) for production, rub .; and - the unit price exclusive of VAT, rub .; $\mathrm{x}$ - planned volume of sales of products of pairs; a0 - the amount of APP; a1 - FÁS amount per unit of product, rub.

Financial strength (PD) shows how much you can reduce the volume of production, working without any loss, without giving a profit, but not suffering losses:

$$
3 \phi=\frac{B-T \delta \cdot y .}{B} \cdot 100(\%)
$$

where Tbu- break-even point.

When calculating the dimensionless enterprises competitiveness indicator estimates the formulas 18 and 19 using the software there is a need to formulate these same criteria as their evidence. For example, the 


\begin{tabular}{|c|c|c|c|c|c|c|}
\hline \multirow{4}{*}{ Impact Factor: } & ISRA (India) & $=3.117$ & SIS (USA) & $=0.912$ & ICV (Poland) & $=6.630$ \\
\hline & ISI (Dubai, UAE & $=0.829$ & РИНЦ (Russia & $=0.156$ & PIF (India) & $=1.940$ \\
\hline & GIF (Australia) & $=0.564$ & ESJI (KZ) & $=8.716$ & IBI (India) & $=4.260$ \\
\hline & JIF & $=1.500$ & SJIF (Morocce & $=5.667$ & OAJI (USA) & $=0.350$ \\
\hline
\end{tabular}

profit per unit of output depends on the profitability of production, that is, first formulated margins from 5\% to $25 \%$, and then laid the size of a unit of production profits. A similar feature exists with the definition of productivity criterion, because at first use innovative processes, formed on the basis of universal and multifunctional equipment maintenance which should be highly trusted and responsible executives, empathic for the overall result of the entire production cycle, guaranteeing them the production of marketable and competitive products, enjoyed by consumers of domestic markets in high demand. Calculation of fixed costs per unit of production and semi-variable costs per unit of output is interconnected with the peculiarities of the organization of competitive and marketable products, including for children. Performance analysis of the leading foreign manufacturers confirms the fact that, if fixed costs are $20 \%-40 \%$ of the cost of production, then, of course, conditional instance variables costs $-60 \%-80 \%$.If this again need to focus on the production of feature products for children, and when profit margins, fixed costs and semi-variable costs are generated based on the implementation of the requirements of technical regulations and normative documents and acts to ensure their safety when using them life. And if this is due to the need of their production with stringent performance - the state and the producers have to be interested in each other, and to provide producers compensation for the additional costs of compliance and the assurance that manufactured products do not cause harm to children's health.

Of course, if the criteria for loss of wages per unit of output has to go to zero, and the volume of the shoe with a $1 \mathrm{~m} 2$ - to its maximum possible value, and the cost of 1 commodity products ruble shall endeavor to achieve the minimum possible value and the cost of equipment per unit of flow setting also aspire to the minimum possible value and other criteria - to their maximum possible znacheniyu- together dimensionless performance evaluation developed innovative technological processes $(\mathrm{K})$ should always page acquainted unit and thereby always confirm that designed an innovative process for the production of the company they import-substituting products will be successful in their work for the benefit of the population of the regions where they operate,

The authors managed to develop software that allows a search to be justified and effective and will help you find the best solution (Table 10) [11-12].

Table 10 - Calculation of the main indicators of the cost of the product range of footwear

\begin{tabular}{|l|l|l|l|l|}
\hline $\begin{array}{l}\text { Model } \\
\text { Indicator }\end{array}$ & $\begin{array}{l}\text { Winter boots } \\
\text { (model A) }\end{array}$ & $\begin{array}{l}\text { Autumn shoes } \\
\text { (model B) }\end{array}$ & $\begin{array}{l}\text { Spring shoes } \\
\text { (Model B) }\end{array}$ & $\begin{array}{l}\text { Summer Sandals } \\
\text { (Model D) }\end{array}$ \\
\hline Profit (RUR). & 245.52 & 194.20 & 83.81 & 69.09 \\
\hline Margin (\%) & 22,11 & 21.40 & 15.19 & 13.18 \\
\hline $\begin{array}{l}\text { Costs per ruble of commodity } \\
\text { products (rub.) }\end{array}$ & 174.71 & 82.37 & 86.81 & 88.35 \\
\hline Semi-variable costs (RUR.) & 839.86 & 599.25 & 291.69 & 271.71 \\
\hline $\begin{array}{l}\text { Conventionally fixed costs } \\
\text { (RUR.) }\end{array}$ & 270.55 & 308.24 & 260.09 & 252.42 \\
\hline The breakeven point (vapor) & 13182.81 & 14923.22 & 22606.93 & 21959.73 \\
\hline Financial strength (\%) & 47.57 & 46.15 & 21.33 & 15.85 \\
\hline Sales (rub.) & 34096215.78 & 30532236.66 & 18264314.24 & 12127790 \\
\hline Gross revenue (RUR). & 6721390.01 & 30532236.66 & 17046769.92 & 2242062 \\
\hline Net income (rubles). & 5229241.43 & 23754080.12 & 13262387,00 & 1744324 \\
\hline
\end{tabular}




\begin{tabular}{llllll} 
& ISRA (India) $=\mathbf{3 . 1 1 7}$ & SIS (USA) & $=\mathbf{0 . 9 1 2}$ & ICV (Poland) & $=\mathbf{6 . 6 3 0}$ \\
Impact Factor: & ISI (Dubai, UAE) $=\mathbf{0 . 8 2 9}$ & PUHL (Russia) $=\mathbf{0 . 1 5 6}$ & PIF (India) & $=\mathbf{1 . 9 4 0}$ \\
& GIF (Australia) $=\mathbf{0 . 5 6 4}$ & ESJI (KZ) & $=\mathbf{8 . 7 1 6}$ & IBI (India) & $=\mathbf{4 . 2 6 0}$ \\
& JIF & $\mathbf{1 . 5 0 0}$ & SJIF (Morocco) $=\mathbf{5 . 6 6 7}$ & OAJI (USA) & $\mathbf{0 . 3 5 0}$ \\
\hline
\end{tabular}

Proof of their assumptions, the authors confirmed the results of the calculation of technical and economic parameters with the help of software developed by them, which allowed us to choose the volume of production, which would be guaranteed by the manufacturer to provide such technical and economic parameters in which to evaluate their comprehensive performance indicator $(C p)$ It will strive to its maximum value, namely, to unity.

As is known, in the period of economic restructuring in our country in the 90 s of the 20th century, the shoe companies to cut production of children's shoes as the unprofitable. However, later on becoming the market of light industry products has shown that this decision was a mistake. As demand grows, and can be seen in the number of children's shops, and exhibitions of footwear products. As a rule, "child" stands are always a lot of customers. The Russian market of children's shoes shows a steady annual growth of $7 \%$ in terms of value. There have been qualitative changes in customer preferences, product requirements are increased.

To date, the world situation is that the production and sale of children's products are profitable. Besides the fact that children are known to be intended "all the best", and there is an objective factor in the high yield of the market: the child is growing, his interests changed, so that clothes and "Toy park" have to be updated almost every season. According to market research AUP.Ru, modern market of children's goods - one of the most dynamic: its annual growth rate of $15-25 \%$. The highest indicators of production and sales volumes provide the speed of its main segments - children's clothing and shoes, toys, food [1]. Therefore, the demand for children's products are not so dependent on the prices and family income level: children's clothes, toys, books require constant updates and ensure that the market turnover.

Demand for children's products in Russia has been gradually increasing since the late $90 \mathrm{~s}$, which explains the increase in the birth rate and improved quality of life. A significant number of children are born in families today have held the "middle class." This is an increase of mass demand and supply. In the market of children's products, in addition to worldclass players, activated domestic manufacturers, opens up many sales outlets and specialty stores where you can find everything - from baby clothes to furniture.

Currently, the number of companies engaged in the production of children's shoes Russia, is constantly growing. A number of enterprises have resumed baby line. They appear and the companies involved had previously only men's and women's shoes. The activities of any modern enterprise is effective when it produces the product or service provided by them are in demand in the market and the satisfaction of certain needs of customers through the acquisition of a product or service makes a profit. [2]

In this regard, and manufacturers, and trade have to take into account two main aspects of the preparation of children's goods to the realization: assortment policy and the quality of products based on anatomical and physiological requirements.

Upon accession to the WTO assortment characteristic of the goods and the quality of their performance will be a significant impact on the demand for products of domestic production.

Therefore, firstly, it is important to match the product to modern trends of children's fashion footwear. Appear more and more new brands, they jostle the old brands, competition is increasing.

In today's market the products of children's shoes as the expensive European brands and cheap footwear from South-East Asia.

China produces about 9 billion pairs of shoes annually, accounting for about $65 \%$ of the total number of shoes produced in the world. However, this situation has changed in recent years. Chinese "Shoemakers" faced with a reduction in demand from European and American countries, and the treatment of their interest to the countries of South-East Asia, placing production in Indonesia or Vietnam. This is associated with lower labor costs in other countries, and to the appreciation of the Chinese raw material. However, as long as China still remains the largest shoe manufacturer in the world.

Currently, the Russian market of footwear are five major price segments. The price range is large enough. In the lower price segment of one pair of shoes cost less than a thousand rubles, while in the premium segment one pair manages more than 7.5 thousand. Rubles. The largest number of purchases in Russia falls on medium-low and medium-price segments of the average shoe. They, in turn, is oriented with the majority of domestic manufacturers. These segments are developing very rapidly, and sales here are actively growing.

In the segment of medium and high-end footwear world leader it is considered the Italian footwear industry, and Italian shoemakers are actually recognized by legislators of the world of fashion footwear. In terms of production of footwear Italy ranks 3rd in the world after China and Brazil.

The history of the formation in Russia of branded footwear market began in the mid-90s, when the country came manufacturers from Italy, Spain and Germany. These countries, and to this day determine the trends in the development of design and comfort of children's shoes.

A few seasons in the children's shoe fashion is almost infinitely dominates sporty:. Krossovochnye, numb the toe and the heel of the sole, bright contrasting inserts and strips, fasteners "fasteners" and lacing, rep edging, etc. In recent years, with it 


\begin{tabular}{|c|c|c|c|c|c|c|}
\hline \multirow{4}{*}{ Impact Factor: } & ISRA (India) & $=3.117$ & SIS (USA) & $=0.912$ & ICV (Poland) & $=6.630$ \\
\hline & ISI (Dubai, UAI & $=0.829$ & РИНЦ (Russia & $=0.156$ & PIF (India) & $=1.940$ \\
\hline & GIF (Australia) & $=0.564$ & ESJI (KZ) & $=8.716$ & IBI (India) & $=4.260$ \\
\hline & JIF & $=1.500$ & SJIF (Morocco & $=5.667$ & OAJI (USA) & $=0.350$ \\
\hline
\end{tabular}

competes etnostil, who introduced usage denim, interesting applications, fringe, embroidery, braiding, proderzhki, stones, etc.

Therefore, the shoe outlines have changed somewhat: if earlier it was like running shoes or sandals for surfing, now in the course of loafers, pumps, shoes and boots with their configuration bertsami, loafers, high boots with a zipper.

Also in children's fashion, we see elegant ballet flats, are fixed to the leg by means of straps, bands or ribbons. They can be stitched not only trim satin but skin and relevant colors.

Under the influence of an adult fashion arsenal of young fashionistas were open mules and clogs decorated in the style of oriental slipper Bakey, ribbons, sequins and rhinestones. Also popular embroidery and appliqués in the shape of hearts, flowers and butterflies. An important focus is the bottom and the details. Since the insole must not be less vivid and expressive than the top. They can be made of leather or textile bright and decorated pattern.

In fashion Printed fabric and leather. Plots are well known - the heroes of the popular comics and cartoons. For special occasions will again be used patent leather, but unlike past seasons with a predominance of black and burgundy, has been a priority of light colors: white, pink, silver in the girls' shoes, blue and blue - boy-in.

It is worth noting the appearance of a new, but very promising tradition - the creation of a common wardrobe style parents when children are dressed in the same clothes and shoes, in which the parents themselves might feel modern and comfortable. There was even a model children's shoes, it is very reminiscent of the adult: a small but pronounced heels, exquisite furniture, elegant materials (lacquer, textured leather with embossed nubuck leather with a metal-plated, etc.). Tops winter boots for girls really like their mothers, decorated with fluffy furry, applications of mink fur, buckles and chains with rhinestones.

Another important trend in children's fashion footwear - the desire for maximum comfort. Use all: design solutions, advanced materials, advanced technology. Among the latest - the new generation mezhpodkladochnye membrane Sympatex High 2 Out, do not absorb moisture inside the shoe, but it allows you to "breathe" the foot, clad in a woolen sock. It is worth noting that in the shoes and boots equipped with this miracle of science and technology, we can make the lining of fur.

The use of children's shoes, and thermostats fiber Outlast, depending on the situation, increasing or reducing the temperature inside the footwear. Another novelty - thermoactive sole Double Fit- System, the heat receiving convenient stack configuration.

Actively used and new design development: anatomical insoles, heel cushions, shock absorbers, bumpers - hard rubber lining on the forefoot.
Vpornosti problem (as you know, chubby baby legs is sometimes difficult to find the right shoes) successfully solved with the help of know-how - to each pair of attached triple set loose insoles of varying completeness.

Very positive, that is rounded, getting comfortable shape, forefoot.

As for koloristki, along with the practical dark colors: black, brown, gray, blue, relevant vibrant colorful shades of red, green, yellow, orange. In favor of white and beige, blue and pink.

Very fashionable combined models, and it's not just about color combinations, but also the use of a single model of materials of different textures (split leather and smooth facial skin, nail and suede, leather, embossed reptile and Cavallino).

In the girls 'actual models lace redundant skin or textiles, in the boys' - mesh or textiles in conjunction with the "breathing" synthetics.

In wet rainy weather rubber boots or comfortable shoes with soles of waterproof materials, rubber, rubber, nylon and others. This trend in fashion is very important. It has long had such a variety of design solutions. They are so "fascinated" consumers that are trying this shoes to wear, even in dry weather. However, this shoe has a low air permeability, so it is only necessary to operate the insoles with good sweat absorbency.

By studying modern range of children's shoes we are constantly confronted with the facts of the close relationship of design and anthropo-physiological requirements of the product. Therefore, this group of factors also requires careful consideration.

Options for high-quality children's shoes on the set of today's Russian market, for every taste and budget. And to ensure that the company has developed successfully, requires regular monitoring of information about the components of the formation range.

High price segment, is inferior in terms of other segments, but, nevertheless, is categorized as stable. It is mainly present foreign producers: Germany (Elefanten, Romika, Ricosta), Spain, Italy, France, Britain and other countries.

Their products are more expensive, but perfect. It utilizes the most advanced know-how and hightech: breathable materials, cushioning system, anatomical sole, thermostats. Most of these companies operate in the Russian market for a long time, and has its own target audience.

At the time that flooded our markets from overseas flow of beautiful and fashionable children's shoes for the most part did not have certificates of conformity, not to mention the hygiene certificates. The large volumes of imported shoes were imported from materials that are harmful to the child's contact with the foot and footwear, according to its design characteristics not corresponding national standards. 


\begin{tabular}{|c|c|c|c|c|c|c|}
\hline \multirow{4}{*}{ Impact Factor: } & ISRA (India) & $=3.117$ & SIS (USA) & $=0.912$ & ICV (Poland) & $=6.630$ \\
\hline & ISI (Dubai, UAI & $=0.829$ & РИНЦ (Russia & $=0.156$ & PIF (India) & $=1.940$ \\
\hline & GIF (Australia) & $=0.564$ & ESJI (KZ) & $=8.716$ & IBI (India) & $=4.260$ \\
\hline & JIF & $=1.500$ & SJIF (Morocco & $=5.667$ & OAJI (USA) & $=0.350$ \\
\hline
\end{tabular}

In connection with this increased percentage of abnormalities in children.

Poor shoes leads to fatigue of the lower limbs, feet and legs pain, swelling soft tissue stop, a stop joint instability under load (e.g., podvertyvaniyu foot during walking).

Numerous observations of doctors confirm that properly designed and matched shoes not only impede the implementation of the normal locomotor functions of the foot, but also causes serious physiological changes in the body, as the musculoskeletal system of children and adolescents in the process of formation and is extremely sensitive to negative influences outside.

Stop child at an early age is very different from the adult foot on the anatomical and physiological structure. In view of the above, to date, the country's urgent need to provide high-quality children's shoes.

Doctors say that children up to 6 years on the foot have body fat, because of which the child does not feel pain when wearing tight shoes, so it's important to pay attention to anthropometric and biomechanical parameters in the selection of children's shoes.

Footwear from the hygienic point of view should protect the body from cooling and overheating to protect the foot from mechanical damage, to assist the muscles and ligaments to hold the arch of the foot in its normal position, providing a favorable microclimate around the foot, help to maintain the required temperature and humidity under any environmental conditions.

Shoes should be light, comfortable, does not restrict movement, conform to the shape and size of the foot. Then the toes are placed freely. For a child's foot is characterized by radial form, in which the maximum width is marked on the ends of the fingers.

The tight and short footwear complicates gait, the foot compresses, blood circulation, causing pain and, over time, changes the shape of the foot, it violates the normal growth deforms fingers, promotes the formation of difficult to heal ulcers, and in the cold time of year can lead to frostbite.

Of course, children are not encouraged to walk in the narrow shoes. Wearing it often leads not only to a curvature of the fingers, the formation of blisters, but also contributes to the development of flatfoot.

Flatfoot is observed during prolonged walking in shoes without heels. Shoes with high (greater than 4 $\mathrm{cm}$ ) heel walking difficult, shifting the center of gravity forward. The emphasis is transferred to the fingers. Dramatically decreases the area of support and stability. The trunk is deflected backwards. This deflection, in an age when the bones of the pelvis is not fused, causing a change in its form, changes the position of the pelvis, which health can affect in the future. This forms a large lumbar flexion. The load on the forefoot is in this case more than 7-fold than in the posterior part). Heel - to artificially increase the arch of the foot, increasing its spring protects the heel from injuries on the ground, and also increases the durability of shoes. By relying on the unshod foot (without spikes), most of the burden falls on the rearfoot. The lack of heel is permitted only in shoes for infants (booties) until the child can not walk. The shoe with a heel $\mathrm{cm} 2$ the load is distributed evenly between the front and rear foot department. Too loose shoes are also harmful. Walking in it quickly tires, and there may be frayed, especially in the field of lifting.

Hygienic requirements for footwear for children and teenagers are made up of the requirements for the design of shoes, due to the peculiarities of the structure of the foot during the growth period, and the materials used for production of shoes. Size, style and rigidity bottom of children's shoes should not hinder the development of the foot. The main elements are cut shoe upper parts - a toe, midsection, Berecz and ankle; and the bottom - is the sole insole, heel.

Since the child is doing on the day of the order of 20 thousand steps, his shoes should be durable and easy, the most flexible and at the same time resistant to wear. For children from 3 to 6 ex-years required tough heel and toe cap. The first - to protect from external influences, the second - for stability. It is also important to maintain the use of the instep of the foot arch.

Models for children should be wide enough, as already noted, in the forefoot, and easy to fasten. Convenient to assume fastener "Velcro" and lightning. A less convenient and the laces buttons. Undesirable presence of longitudinal seams on the vamp in the shoes and boots.

The sole should bend well. The rigid sole impedes walking (bending angle is limited, the shoe heel with the heel shrinks), reduces the performance of ankle muscles, increases skin temperature and perspiration legs.

As far as is necessary to ensure maximum mobility of the forefoot, so it is necessary to ensure maximum stability of the heel. The back should be firm, non-slip feet, tightly cover the heel, to prevent its deformation.

Stop child can easily be deformed under the influence of mechanical stress. In this regard, such qualities as thickness, flexibility of the sole, shoe weight and thermal insulation properties are subject hygienic norms.

For infant shoe upper leather is recommended because it has a high air and water vapor permeability, softness, flexibility and heat-shielding properties.

Footbed - internal parts of footwear, which has contact with the skin of the foot and promotes a comfortable temperature and humidity conditions during vnutriobuvnom space. It must have a high air and water vapor permeability. It should be made only from genuine leather.

Children's shoes should have a safe and convenient fastening on leg, do not hinder movement. 


\begin{tabular}{|c|c|c|c|c|c|c|}
\hline \multirow{4}{*}{ Impact Factor: } & ISRA (India) & $=3.117$ & SIS (USA) & $=0.912$ & ICV (Poland) & $=6.630$ \\
\hline & ISI (Dubai, UAE & $=0.829$ & РИНЦ (Russia & $=0.156$ & PIF (India) & $=1.940$ \\
\hline & GIF (Australia) & $=0.564$ & ESJI (KZ) & $=8.716$ & IBI (India) & $=4.260$ \\
\hline & JIF & $=1.500$ & SJIF (Morocce & $=5.667$ & OAJI (USA) & $=0.350$ \\
\hline
\end{tabular}

To do this, use different types of fastening: lacing, "Velcro", straps, buckle with a zipper, etc..

For children's shoes allowed thread and combined methods of fastening, providing more flexibility in beam area, ease in applying foam rubber, polyurethane and others. Materials. Uses adhesive and molding methods of fastening, ensuring waterproof shoes, which is especially necessary in the spring and autumn-winter periods.

The consequences of poor quality shoes is much more serious than on clothing. It is this tendency has identified the current state of children's shoes market. She stopped to save.

Increasing the volume of the release of goods for children is of great social, economic and political importance, both for the country as a whole and for the individual region.

It is therefore important to monitor the situation in the regions. Of particular interest is the Siberian region.

To analyze the quality of the shoe, and obtaining a sufficiently realistic picture of the market of children's shoes it is advisable to focus on the results of a major regional commercial and industrial enterprises. As such an object has been selected Ltd. "UK SCC Monroe", which is one of the largest footwear companies in Russia - both in terms of sales of shoes and latitude range presented.

Today the company has two wholesale divisions in Novosibirsk and Moscow, and more than 130 retail stores in Novosibirsk, Tomsk, Novokuznetsk, Kemerovo, Barnaul, Chelyabinsk, Perm, Ufa and other cities. Group trading companies "Monroe" for more than 14 years of experience in the development and implementation of collections of shoes. The company is headquartered in Novosibirsk.

Footwear significantly affects the quality of its implementation. An important feature of the shoe division defects are the place and their causes, the degree of importance, and the ability to detect and eliminate. By birth defects are divided into six main groups: materials; top preform assembly; molding preforms; attaching the bottom; finishing of footwear; packaging, labeling and storage. By operating the various defects include damage arising in step socks and shoe care. Defects in operation are usually subdivided into a bottom part defects and their fastenings, defects top parts, pads and braces [].

Defects are measured by their effect on the ability of the shoe perform specified functions in accordance with the purpose. On the grounds of defects are divided into functional and non-functional. Recent violate the proper performance of the functions of footwear, so depending on the product conditions, wear functional defects may be avoidable and unavoidable.

Defects in the children's shoes are not only degrade the performance properties, but also may adversely affect the development of children's feet. We have analyzed the most common in retail stores defects of children's footwear company "UK SCC Monroe." The following defects have been identified. The most common manufacturing defects are different height tops (30\%), the folds of the lining (22\%), offset rear outer belt $(15 \%)$. Less common defects such as a different height tibia $(5 \%)$, the dye migration $(3 \%)$, the deformation of the toe cap $(3 \%)$ and otkleyka padding (2\%) (Figure 10).

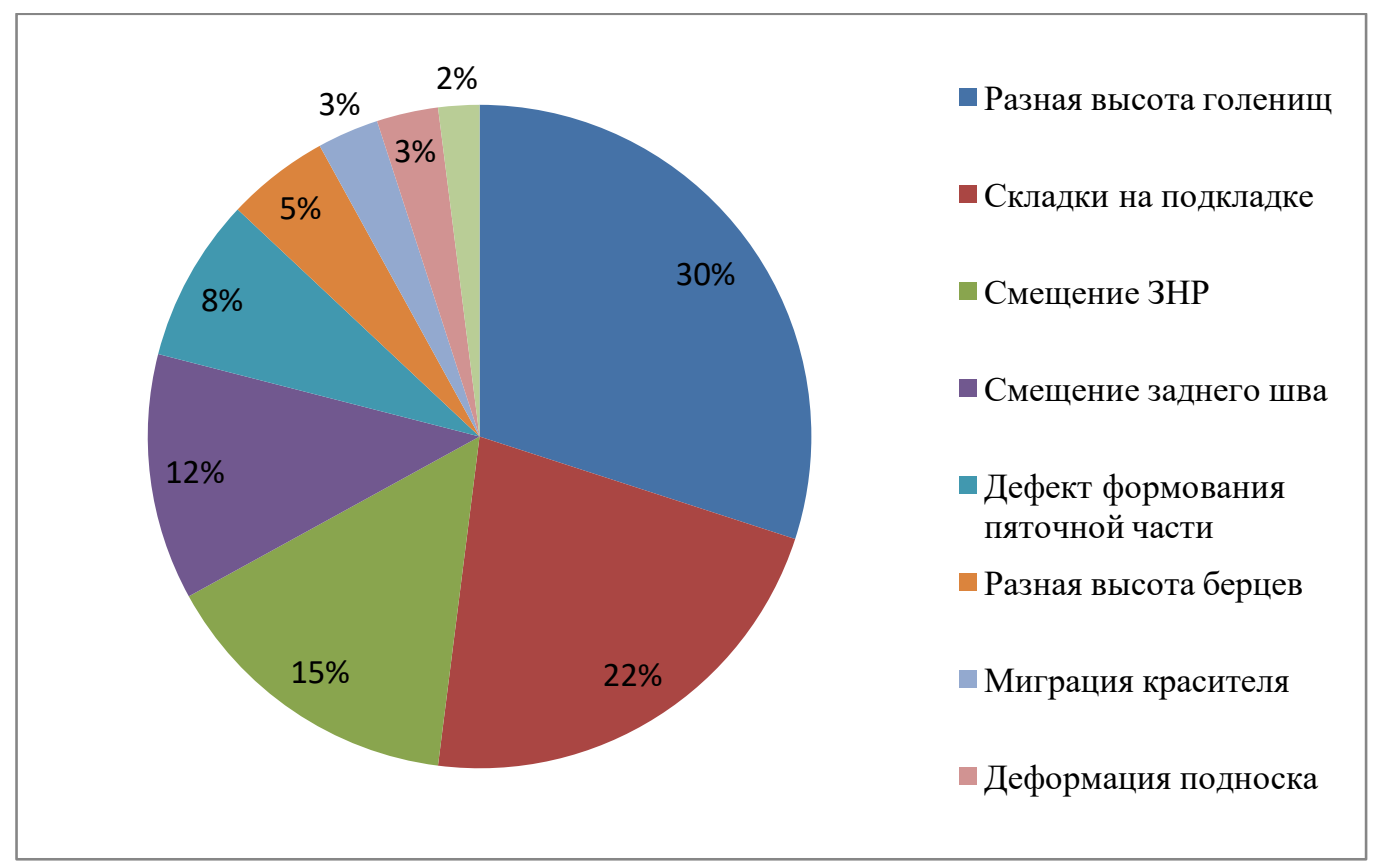

Figure 10 - Diagram "Manufacturing defects" 


\begin{tabular}{|c|c|c|c|c|c|c|}
\hline \multirow{4}{*}{ Impact Factor: } & ISRA (India) & $=3.117$ & SIS (USA) & $=0.912$ & ICV (Poland) & $=6.630$ \\
\hline & ISI (Dubai, UAE & $=0.829$ & РИНЦ (Russia) & $=0.156$ & PIF (India) & $=1.940$ \\
\hline & GIF (Australia) & $=0.564$ & ESJI (KZ) & $=8.716$ & IBI (India) & $=4.260$ \\
\hline & JIF & $=1.500$ & SJIF (Morocco) & $=5.667$ & OAJI (USA) & $=0.350$ \\
\hline
\end{tabular}

It should be noted that the most dangerous for the child's foot is a defect in the folds of the lining. In autumn shoe lining - it's villi. But the most dangerous in the folds of winter shoes, because it is more dense material Lining - fur, and therefore, folds rougher. And in fact, and in another case, the folds being rubbed leg, damaging the skin, blisters appear. Moreover, excessive pressure on the foot in the area of deformation adversely affects its development, as the child's body is not resistant against external influences. Excessive pressure can lead to pinched vessels, their anemia, and prolonged exposure - to a change of the foot skeleton.

Post- defects (step sale): most common material such as upper defect contamination $(60 \%)$, raznoton top material (20\%), breakage of fittings (8\%). Less common defects such as a gust of padding (6\%), adjustable tachnogo weld defect (4\%) and mechanical damage of the top material (2\%) (Figure 11).

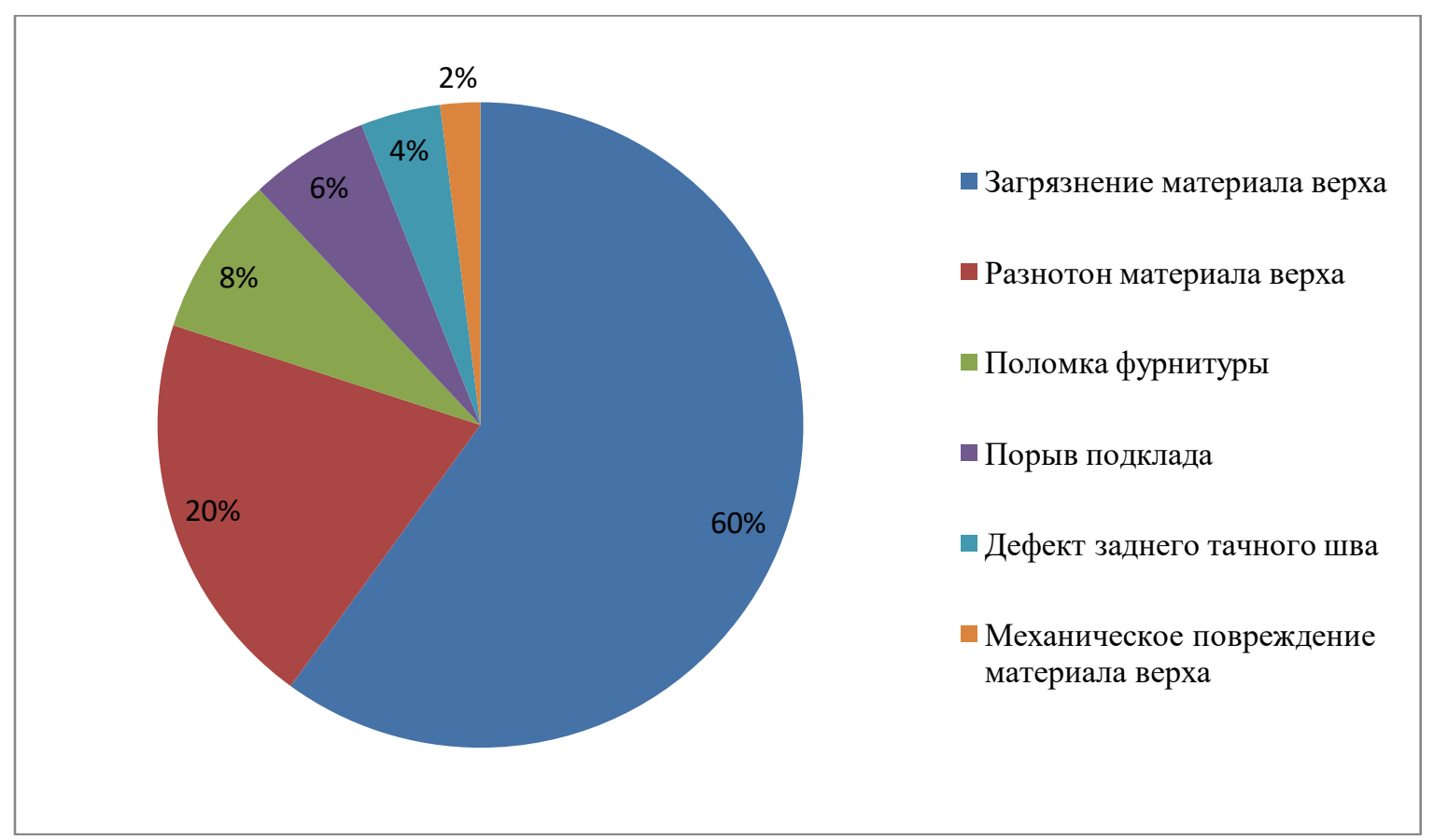

Figure 11 - Diagram "post- defects"

A set of footwear production quality and design decisions that affect the usability and aesthetically pleasing appearance, determines the price niche product realization.

Today's footwear manufacturers and distributors operate in all market segments, from low to high. Nielsen study reports that in the largest Russian cities, parents spend on children's products from 3100 to 4600 rubles. per month, and more than a third 'child' budgets are children chain stores. Of course, the cost of those who prefer the products of luxury brands is much higher. Experts point out that, not face a shortage of buyers of a network operating in the premium segment, but opportunities in this segment are limited. So players premium are now going in the medium price range, and vice versa.

According to experts, in the next few years, the Russian footwear market will maintain growth trends.

The Russian manufacturers new opportunities. Now comes the process of creating a new Russian footwear production and the simultaneous formation of shoe retailers. In recent years, the shoe retail is growing very rapidly: the number of stores within the network for the year increased by half to two times. Sales geography - all regions of Russia.

As a result, the majority of entrepreneurs operating on the territory of Russia, employs identification and selection of proper trade size.

The average price niche (about 30\%) is represented mainly footwear Russian manufacturers, because this segment of the market has a significant turnover from the sale. As already mentioned, this fact is due to the growth of material well-being of customers and their preferences shift towards higher quality products, for which they were prepared to pay.

For example, the Russian market of children's goods begins to intensify in recent years with the emergence of new stores average price level, which combine commerce with entertainment.

The difficulty of the organization of trade in this market segment is that the "child" Retail requires a large range because each age group needs a completely different products, which in turn leads to the need for large sales areas and complex logistics. Children's shoes to make and sell is very difficult - the production costs almost the same as in the adult shoes and the price should be lower. In addition to the 


\begin{tabular}{|c|c|c|c|c|c|c|}
\hline \multirow{4}{*}{ Impact Factor: } & ISRA (India) & $=3.117$ & SIS (USA) & $=0.912$ & ICV (Poland) & $=6.630$ \\
\hline & ISI (Dubai, UAI & $=0.829$ & РИНЦ (Russia & $=0.156$ & PIF (India) & $=1.940$ \\
\hline & GIF (Australia) & $=0.564$ & ESJI (KZ) & $=8.716$ & IBI (India) & $=4.260$ \\
\hline & JIF & $=1.500$ & SJIF (Morocco & $=5.667$ & OAJI (USA) & $=0.350$ \\
\hline
\end{tabular}

children's shoes size range is several times larger than in adults.

In addition, the organization "Children's" Retail has its own peculiarities - such shops must be, so to say, spectacular. children's shop visitors (and usually purchases made with your family) need not only goods, but also the possibility of something to take the child, so the sale is forced to create children's corners and come up with all sorts of entertainment, which require additional space, and do not bring profit.

Thus, we see that the new format retail chains gradually comes to the replacement market, which are formed in the low and middle price segments. In the high-price niche boutiques and specialty shops are being implemented branded products. In this case, there is a marked lag the regional markets of the capital region. This factor should be taken into account children's range in the Siberian region for trade planning.

Formation of the order necessary range associated with the study of consumer preferences. To develop high-quality and popular shoes often use marketing methods.

In marketing, research is important, because in a market advantage is given to those firms and companies who know better than anyone the needs of consumers and produce products that can satisfy them. But as the market is constantly changing, the needs of the people under the influence of various factors are also changing. Therefore the firm to make a profit, have to constantly monitor the market situation. It is with the help of market research company can carry out monitoring of the change in consumer needs.

Currently, the most popular method for collecting primary data is polling method. The survey is a very effective way to obtain information as a universal objective (on the facts of human activity) and subjective (the motives of activity, opinions, estimates and value orientations).

The survey - a method of direct (interviews) or indirect (profile) collection of primary verbal information by recording the answers to the questions, grouped in the form of a questionnaire in accordance with the purposes and objectives of the study.

Questionnaire - one of the main types of survey, which involves the construction of rigidly fixed order form. Questions in the questionnaire should be clearly articulated, understood the respondent, as well as contain a list of options.

The peculiarity of the questionnaire is that the respondent is working independently with a questionnaire, that is understood to be mulling and answers the question, in accordance with their knowledge, beliefs, values.

Questionnaire - a tool is very flexible in the sense that the issues can be defined in many different ways. During the development of the questionnaire the researcher selects questions that should be asked, choose the form of these questions, their wording and sequence. Research questions are structured and take a narrow focus [].

In order to study consumer preferences, requests and needs of the studies were carried out using in retail stores survey of buyers of "Criminal SCC Monroe."

The main objective of the survey - analysis and interpretation of data, summarize the findings and provide recommendations for improvements.

A questionnaire survey was carried out in the retail trade in the departments of children's shoes, as well as on the internet. Respondents were asked to fill out a form with which to seek the views of respondents on a number of issues. The survey was conducted after the purchase that the respondent had a desire to embellish their consumer preferences

The target audience is women and men of all ages, with children and grandchildren up to 16 years. The sample consisted of 310 people, which is sufficient to obtain reliable results.

After processing of personal data The following results were obtained. The majority of respondents women, men make up $12 \%$ of the total, they came into the store with their wives and children. $46 \%$ of respondents were aged between 25 and 30 years, $25 \%$ under $25,12 \% 31-35$ years $10 \% 36-45$ years and $7 \%$ of people older than 46 years (Figure 12). 


\begin{tabular}{|c|c|c|c|c|c|c|}
\hline \multirow{4}{*}{ Impact Factor: } & ISRA (India) & $=3.117$ & SIS (USA) & $=0.912$ & ICV (Poland) & $=6.630$ \\
\hline & ISI (Dubai, UAE & $=0.829$ & РИНЦ (Russia & $=0.156$ & PIF (India) & $=1.940$ \\
\hline & GIF (Australia) & $=0.564$ & ESJI (KZ) & $=8.716$ & IBI (India) & $=4.260$ \\
\hline & JIF & $=1.500$ & SJIF (Morocce & $=5.667$ & OAJI (USA) & $=0.350$ \\
\hline
\end{tabular}

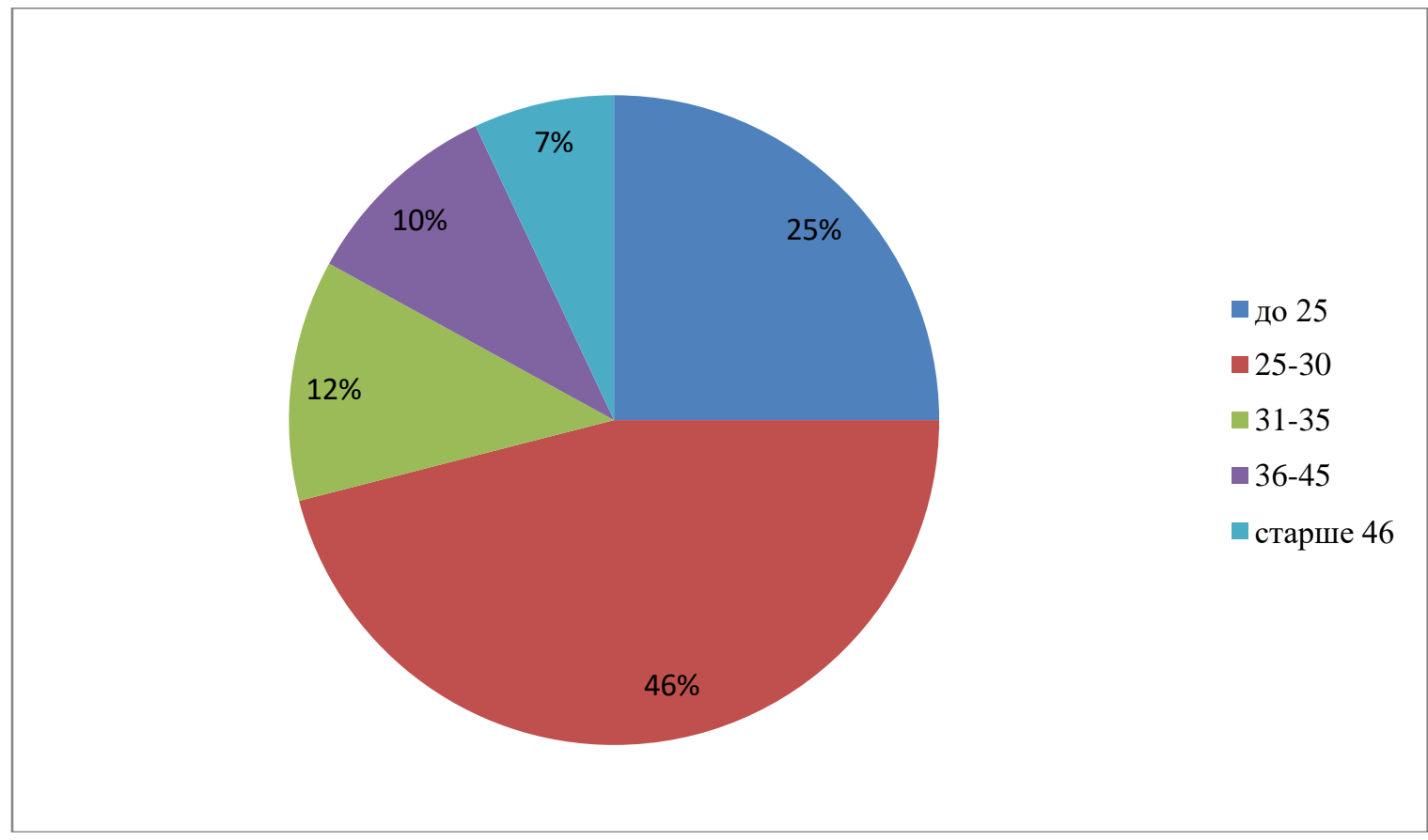

Figure 12 - Diagram "Age of respondent"

Among Ltd. SCC MONROE shoes for preschoolers buy approximately the same number of girls and boys. For school-age children: more girls to the range of models, and boys more and more couples on one model. The number of models for boys bought less than for girls.
Most of the people we surveyed have an income per family member in the amount of 6-10 million rubles ( $35 \%$ of). $20 \%$ of respondents have an income of 11-15 thousand rubles, $18 \%$ - up to 5000 rubles. Income $17 \%$ of $16-25$ thousand rubles, and only $10 \%$ of respondents the income per family member is 26 35 thousand rubles (Figure 13).

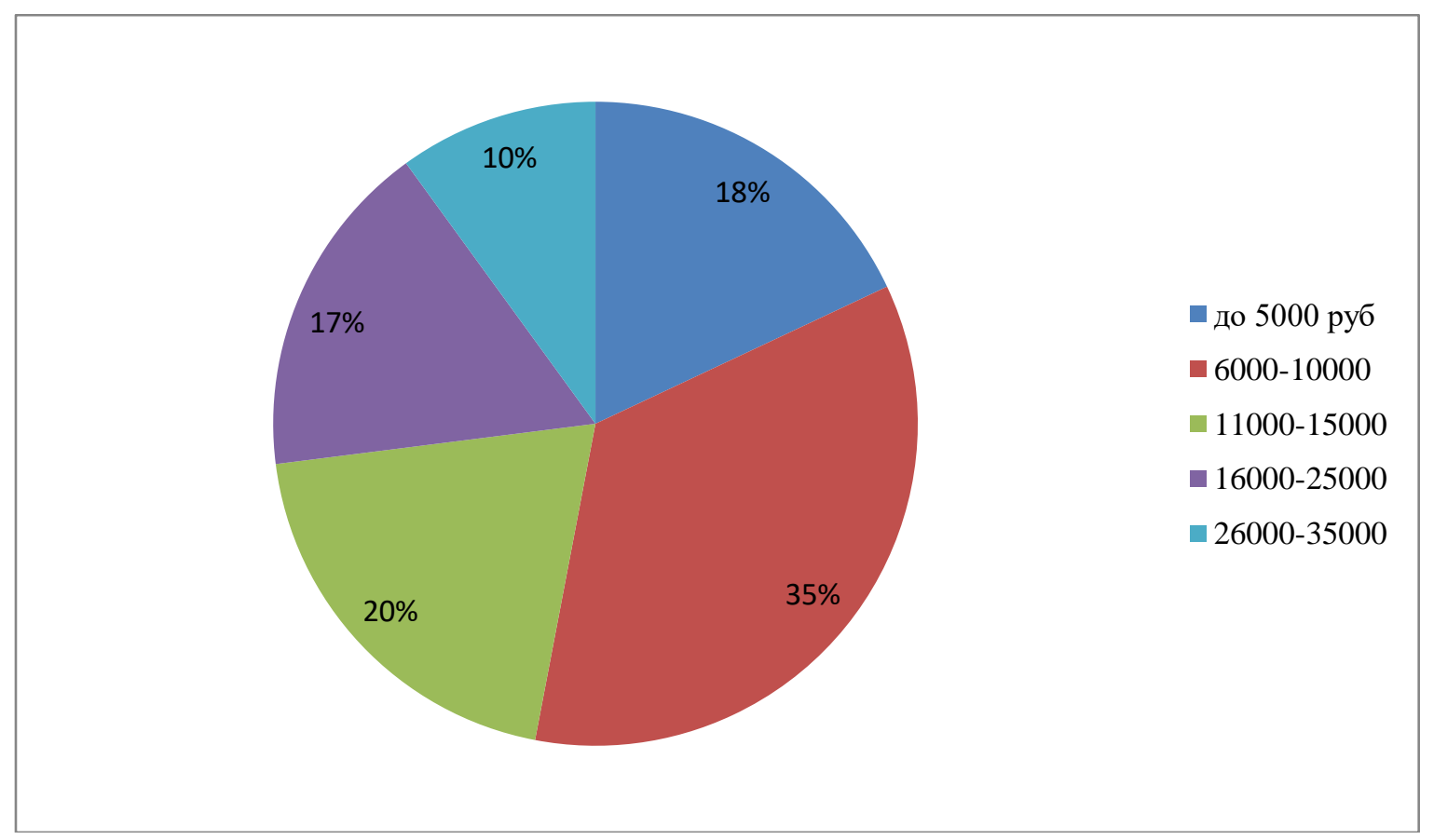

Figure 13 Chart "Income per family member"

It is very important for the implementation of the article is the question of the place of purchase shoes. According to the results of our studies, it was found that people prefer to buy shoes in retail stores and shopping centers (86\%), as an important factor in the choice when buying a quality. Survey respondents 


\begin{tabular}{|c|c|c|c|c|c|c|}
\hline \multirow{4}{*}{ Impact Factor: } & ISRA (India) & $=3.117$ & SIS (USA) & $=0.912$ & ICV (Poland) & $=6.630$ \\
\hline & ISI (Dubai, UAE & $=0.829$ & РИНЦ (Russia & $=0.156$ & PIF (India) & $=1.940$ \\
\hline & GIF (Australia) & $=0.564$ & ESJI (KZ) & $=8.716$ & IBI (India) & $=4.260$ \\
\hline & JIF & $=1.500$ & SJIF (Morocce & $=5.667$ & OAJI (USA) & $=0.350$ \\
\hline
\end{tabular}

doubt the quality of shoes available on the market. Which confirmed the feasibility of the development of chain stores in the direction of Novosibirsk.
Case in point is the study of consumer preferences branded stores and product brands. Consumers could choose multiple brands. Preferences as follows (Table 11.

Table 11 The preferred place to buy children's shoes

\begin{tabular}{|c|c|c|}
\hline Score & Specialization & \% Consumer Choice \\
\hline 1. Monroe & specialized & 79 \\
\hline 2. Fidget & multibrand & 46 \\
\hline 3. Kotofey & specialized & 24 \\
\hline 4. NATI & multibrand & 17 \\
\hline 5. Child's world & multibrand & 13 \\
\hline
\end{tabular}

Interviewed buyers prefer shopping "Monroe" retail network (79\% of). This result is due to high orientation of the company in the Siberian region.

Neposeda (46\%). The main audience of middleand upper-middle-income countries. "Hurry City" presented in the demanding range of customers more than 5000 names of goods from leading mills in Austria, Poland, Russia, China, Turkey and Thailand. Experts are selected shoes and things made of natural materials. And one of the main competitive advantages - a wide choice of products, not only for kids, but also for children from 5 to 14 years.

In recent years, a network of children's shops "Fidget-city" due to its striking advertising campaigns, long remembered by the citizens, has become one of the most recognized in the city, and greatly expanded range of buyers.

«NATI» $(17 \%)$. Customers receive a harmonious blend of innovative technical solutions that support European level of service in the «NATI» stores, unique services designed to provide maximum comfort, use and developing eco-friendly elements. The range in the shops «NATI» selected taking into account the recommendations of modern nutritionists and pediatricians: food, cosmetics, accessories for babies, cribs, strollers, high chairs, clothes, shoes, toys, educational games.

Children's world (13\%). For over 50 years there is a Russian brand "Children's World." During this time inside the network have formed the tradition, allowing you to successfully pass through all the trials of the last century. The combination of tradition and the desire to keep up with the growing demands of the time allowed the "Children's World" to enter a new stage of development - the creation of a nationwide network of kids' store of the 21st century.

Kotofey (24\%). Brand "Kotofey" was registered in 2001, one of the oldest Russian manufacturers of children's shoes "Egorievsk-shoes." Every year more and more children's shoes buyers prefer this brand.

The company pays great attention to the issue vpornosti and compliance of its products orthopedic requirements. Together with the Department "Technology of leather products" of the Moscow State University of Design and Technology is constantly conduct research, mass measurements stop Russian children, the results of which are developed evidencebased pads, taking into account the characteristics of children's feet. New pads will produce a really good shoes, which is very important especially for the emerging children's feet.

A wide selection range and constant updating of the range was reserved for the leading position among the manufacturers of children's shoes. In the year it offered more than 1000 different models.

Consider the preferred brand of children's shoes.

\section{Conclusion.}

Antelope (Russia). In the production line there are all kinds of children's shoes: sneakers, shoes, boots, sneakers, shoes, boots, rubber, cast and exaggerated shoes. The company constantly improves the production technology, focusing on innovations in materials, tailoring equipment, and medical requirements. Children's shoes, "Antelope" is made of genuine leather, environmentally friendly synthetic materials permitted for the production of children's shoes Russian Ministry of Health and were certified.

Bagira (Romania). Due to application of the membrane material, the baby's legs in the shoe trade mark "Bagheera" always have a constant temperature (approximately $31^{\circ} \mathrm{C}$ ), without overheating and not subcooled. Gore-Tex is placed between the shoe upper and lining. Apart from the material Gore-Tex, a "Bagheera" winter shoes outsole consists of PVC, and serves as an artificial fur lining material. The entire range is distinguished by bright, vivid colors that are popular with both children and their parents.

Alligasha (Russia). When you create a product by the manufacturer takes into account all the requirements for children's shoes are not flat sole with a heel binding, quality materials and professional manufacturer. On the quality of shoes is the fact that some of the lines of the model given the status of orthopedic. It takes into account the company and fashion trends, constantly updating its range of products and ensuring that it is always fashionable and in demand.

Kotofey (Russia). The product range is very wide: Shoes for the little ones, textile and removable 


\begin{tabular}{|c|c|c|c|c|c|c|}
\hline \multirow{4}{*}{ Impact Factor: } & ISRA (India) & $=3.117$ & SIS (USA) & $=0.912$ & ICV (Poland) & $=6.630$ \\
\hline & ISI (Dubai, UAI & $=0.829$ & РИНЦ (Russia & $=0.156$ & PIF (India) & $=1.940$ \\
\hline & GIF (Australia) & $=0.564$ & ESJI (KZ) & $=8.716$ & IBI (India) & $=4.260$ \\
\hline & JIF & $=1.500$ & SJIF (Morocco & $=5.667$ & OAJI (USA) & $=0.350$ \\
\hline
\end{tabular}

shoes, shoes, shoes, shoes, sneakers, boots and ankle boots, boots, rubber shoes, and even shoes with the membrane. Much attention is paid to a style of shoes top, because it captures the upper leg in position and prevents injury by careless walking. In the smallest size groups of all footwear is made on the ankle. An ideal locking element is also lacing. Special attention is focused on the manufacturer of the features of children's shoes, like flexibility and toughness backs. "Kotofey" is one of the few companies that manufacture shoes with leather soles, the most ecofriendly for children's feet. Presented trademark manufactures products with minimum weight.

Lel (Russia). The factory assortment more than hundreds of models, designed for all seasons: winter, summer and autumn-spring shoes. This includes small children, preschool and school shoes for boys and girls. The main materials used in the process of "Lel" production of children's shoes, are leather, chrome, naplak, suede and nubuck. The lining is preferably used traspira. Another advantageous feature of the company's products "Lel" - is the perfect combination of quality and price. The range: the shoes, boots, boots, sandals, slippers.

Smeshariki (Russia). Children's favorite characters is applied to absolutely all kinds of shoes. Children's boots "Smeshariki" series are available with a molded outsole - something like the bottom of the boots, but modern sample. It is perhaps a little prevents run and jump, but because the child's foot remains dry. In addition, the shoes are made with fortified backdrop instep and the average height that the child correctly set foot and did not get flat feet. A sturdy heel and ankle support saves baby from injuries during games.

Thomas (Russia). Collection series of the brand "Thomas" is constantly updated with new models casual, everyday, festive, school, sports, children's shoes. The range of models is presented with different attachments: glue-bonding, adhesive, glue, stitch, sandalno-adhesive. In the first place it puts the practicality, reliability and comfort.

Shalunishka (Ukraine). Lineup repositioned three types of shoes: moccasins, sandals and shoes for all seasons. Soft and flexible booties - especially for kids. Shoes meets all the requirements of the footwear for children from toddlers and teens. Has good waterproof and heat-shielding properties, is resistant to abrasion. Used orthopedic insoles for prevention of flat feet and a curvature of the foot.

Unichel (Russia). The company "Unichel" positions itself as a manufacturer of inexpensive and high-quality footwear, which could compete with the Italian model. Assortment - boots and shoes, shoes. "Velcro" - for easy donning. "Unichel" features highquality products.

Loew Sunny, Ulot (Russia-China). They are their own exclusive trademarks SCC Monroe. The range consists of more than one hundred models. Presented shoes for all seasons, different age groups. Highlighted line of athletic shoes. A wide range of species: sandals, slippers, shoes, sneakers, boots and shoes, boots, ankle boots and boots. In the process of making shoes using both natural and artificial materials which are distinguished not only by its versatility for a wide variety of styles, but also highly relevant design [4].

Thus, it is represented by the brand of children's shoes meet all the requirements imposed by consumers to the product.

Among the factors influencing the choice of children's shoes, most respondents selected most significant: comfort $(23 \%)$, price $(17 \%)$, natural top material (14\%). The least significant in this case were such factors as the consultation seller in the store $(7 \%)$, the taste and preferences of the child $(8 \%)$, and trademark (8\%) (Figure 14). 


\begin{tabular}{|c|c|c|c|c|c|c|}
\hline \multirow{4}{*}{ Impact Factor: } & ISRA (India) & $=3.117$ & SIS (USA) & $=0.912$ & ICV (Poland) & $=6.630$ \\
\hline & ISI (Dubai, UAE & $=0.829$ & РИНЦ (Russia) & $=0.156$ & PIF (India) & $=1.940$ \\
\hline & GIF (Australia) & $=0.564$ & ESJI (KZ) & $=8.716$ & IBI (India) & $=4.260$ \\
\hline & JIF & $=1.500$ & SJIF (Morocco) & $=5.667$ & OAJI (USA) & $=0.350$ \\
\hline
\end{tabular}

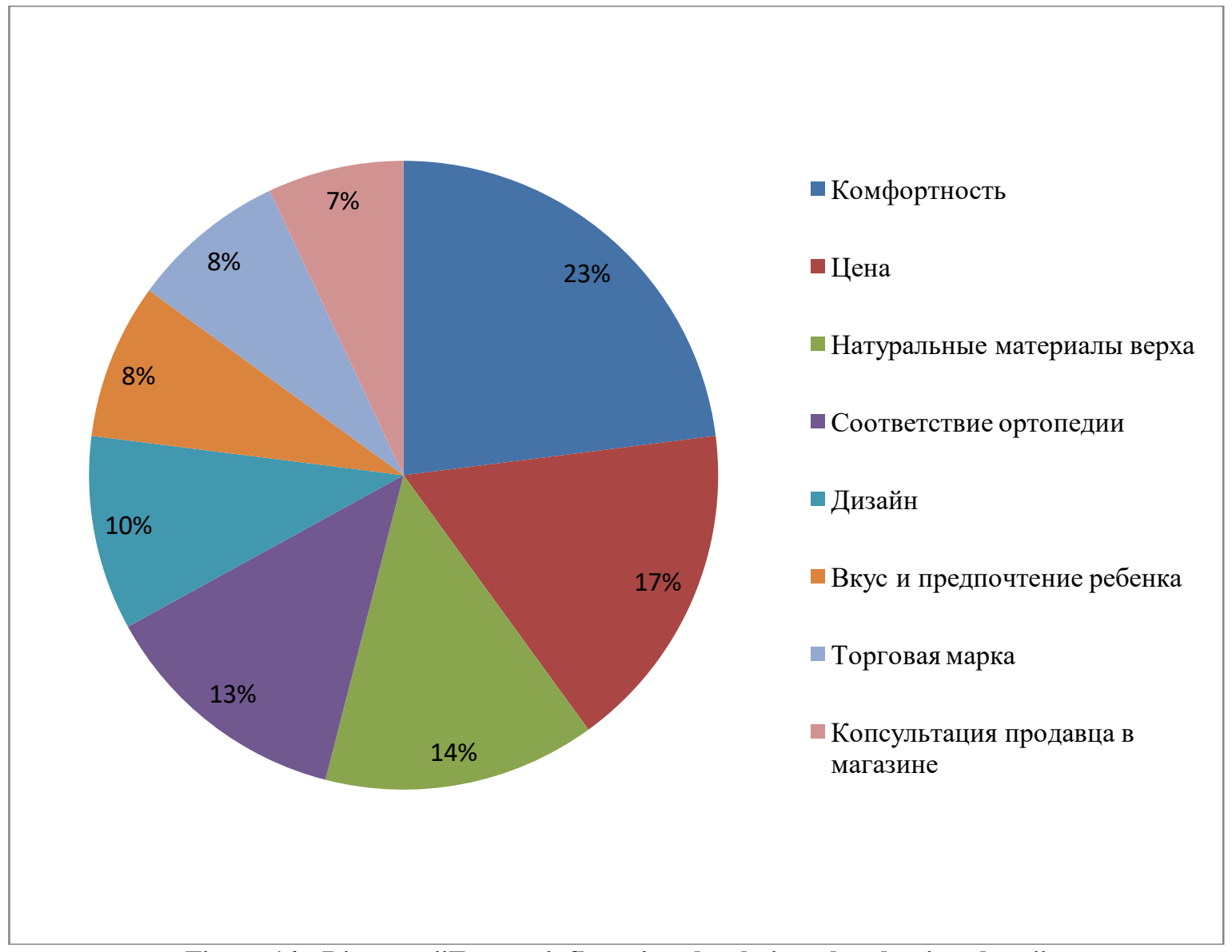

Figure 14 - Diagram "Factors influencing the choice when buying shoes"

Determination of color preferences revealed that consumers prefer to buy shoes of bright colors for children of preschool age, regardless of the season; School demi-season dark colors for both girls and boys. Separately noted that summer footwear prefer color, especially in the sports of sneakers soles.

In the course of our research we found that most are buying shoes at school age 31-37 sizes. When children go to school shoes need more for several reasons: for the summer leg grows; all parents want to September 1st child was dressed and shod in all-new; Also, the school you want to change shoes and shoes for physical education classes; often take to school shoes and shoes for walking.

The number of pairs of shoes purchased for the season, also depends on the age of the child welfare parents. Young children (size 20-25) take a little: sandals (may take 2 pairs per year since the foot is growing rapidly, and the shoes are often even at home on the advice of doctors), a pair of oxfords, a pair of autumn botinochek (or "Pectoral Sandpiper"), one winter . Complement the wardrobe a little secondhand shoes, which is transmitted acquaintances, relatives. Therefore, the actual wardrobe of shoes for children is greater than buying. Sizes 25-30: take a change of shoes to kindergarten, summer shoes, shoes or shoes, demi-season shoes, winter (often still plus boots), rubber boots. Sizes 30-35: Summer shoes, spare shoes to school, school shoes, walking shoes, demi-season shoes, winter shoes, girls more elegant shoes, rubber boots. The girls in this group do not often take one pair of shoes, and 2 or more. It deals more with shoes and summer shoes. Thus, it is revealed that with age, the number of pairs increases.

In the survey respondents said that most children buy shoes because the foot has grown. But the older the child, the more significant are other factors: the boys become more active - shoes deteriorating rapidly; girls start to follow the fashion, to pay more attention to their clothes, shoes - want a new pair of respectively more beautiful and fashionable.

We have found that the ratio of the sizes for 3036 Number girls boots and shoes $60 \%$ to $40 \%$. Preferably bootleg with folds (hides completeness or thinness legs) or with laces, to adjust the width of the ankle. Demand model with knitted inserts on the ankle.

Also during the interview following the preferences of buyers of children's shoes have been identified:

- 23-30 rr without laces, a preferred fastener "Velcro" with the possibility of adjustment; Girls PU Leather (more choice), boys leather (more move, the foot sweats); 


\begin{tabular}{llllll} 
& ISRA (India) $=\mathbf{3 . 1 1 7}$ & SIS (USA) & $=\mathbf{0 . 9 1 2}$ & ICV (Poland) & $=\mathbf{6 . 6 3 0}$ \\
Impact Factor: & ISI (Dubai, UAE) $=\mathbf{0 . 8 2 9}$ & PUHL (Russia) $=\mathbf{0 . 1 5 6}$ & PIF (India) & $=\mathbf{1 . 9 4 0}$ \\
& GIF (Australia) $=\mathbf{0 . 5 6 4}$ & ESJI (KZ) & $=\mathbf{8 . 7 1 6}$ & IBI (India) & $=\mathbf{4 . 2 6 0}$ \\
& JIF & $\mathbf{1 . 5 0 0}$ & SJIF (Morocco) $=\mathbf{5 . 6 6 7}$ & OAJI (USA) & $\mathbf{0 . 3 5 0}$ \\
\hline
\end{tabular}

- 30-36 pp for girls models like adult shoes; without laces, on the clasp "Velcro" pribivnoy heel is not in demand; preferred footwear on a platform sole.

Thus, the analysis of the situation both in Siberia and in the domestic market of shoes in other Russian regions, its footwear saturation showed that in these regions is implemented Russian trend of network trade. Confirmed the growing demand for children's products. These studies suggest that the average price segment of Russian companies compete with foreign manufacturers, as in the production of its products using imported components, technology and, in addition, develop their own sales networks. It identified the most popular brands of children's footwear. The factors influencing the formation range (as the species and size) for different regions of Russia.

\section{References:}

1. Golovko, V. A., et al. (2019). On the possibilities of normative documentation developed within the framework of the system quality management (QMS) for the digital production of defect-free importsubstituting products: monograph / under the General editorship of Dr. of technical Sciences, Professor V. T. Prokhorov (Eds.). the Institute of service sector and entrepreneurship (branch) don state technical University. (p.227). Novocherkassk: Lik.

2. (2018). Managing product quality through motivation behavior of the leader of the group of light industry enterprises: monograph / under the General editorship of Dr. sci. prof. V. T. Prokhorova (Eds.). Institute of service and entrepreneurship (branch) of the don state technical University. (p.336). Novocherkassk: Lik.

3. Prokhorov, T. V., et al. (2013). Synergetics of the formation of a competitive range of domestic footwear: monograph/ under the General editorship of doctor of technical Sciences, Professor V. T. Prokhorov; VoIP (branch) of DSTU. (p.194). Mines: Isoip (branch) DSTU.

4. Surovtseva, O. A., et al. (2018). Management of real product quality and not advertising through the motivation of the leader of the team of the enterprise of the legka industry: monograph / under the General ed. Dr. Techn. prof. V. T. Prokhorova; Institute of service and entrepreneurship (branch) of the don state technical University. (p.384). Novocherkassk: URGU (NPI).

5. Prokhorov, V. T., et al. (2018). Competitiveness of the enterprise and competitiveness of production - guarantee of successful import substitution of the goods demanded by consumers of regions of southern Federal district and skfo. the collective monograph / under the General ed. prof. V. T. Prokhorova (Eds.). Institute of service sector and entrepreneurship (branch) of don state technical University. (p.337). Novocherkassk: Lik.

6. Prokhorov, T. V., et al. (2015). The range and assortment policy: monograph / under the General editorship of Dr. sci. Sciences, Professor V. T. Prokhorov (Eds.). Institute of the service sector and entrepreneurship (Phil.) Fader. state budget. educated. institutions of higher. professional education "don state technical. UN-t" in mines Growth. region (Isoip (branch) of DSTU). (p.503). Novocherkassk: URGU (NPI).

7. Prokhorov, V. T., et al, (2017). The concept of import substitution of products of light industry: background, challenges, and innovations. monograph / under the General editorship of Dr. sci. prof. V. T. Prokhorova (Eds.). Institute of service and entrepreneurship (branch) of the don state technical University. (p.334). Novocherkassk: Lik.

8. Prokhorov, V. T., et al. (2014). Revolution of quality: through the quality of advertising or real quality. the monograph, under the General ed., prof. V. T. Prokhorov (Eds.). Isoip (branch) DSTU. (p.384). Novocherkassk: URGU (NPI).

9. (2012). The impact of cash flow on the efficiency of the cluster, formed on the basis of Shoe enterprises of the southern Federal district and skfo. L. G. Gretskaya [et al.]; under the General ed. (Eds.). (p.354). Mines: FGBOU VPO yurgues.

10. Vdovin, L. P., Prokhorov, V. T., Osin, T. M., Meleshko, E. N., \& Osaca, N. I. (2014). A means of providing sustainable technical 


\begin{tabular}{|c|c|c|c|c|c|c|}
\hline \multirow{4}{*}{ Impact Factor: } & ISRA (India) & $=3.117$ & SIS (USA) & $=0.912$ & ICV (Poland) & $=6.630$ \\
\hline & ISI (Dubai, UAI & $=0.829$ & РИНЦ (Russia) & $=0.156$ & PIF (India) & $=1.940$ \\
\hline & GIF (Australia) & $=0.564$ & ESJI (KZ) & $=8.716$ & IBI (India) & $=4.260$ \\
\hline & JIF & $=1.500$ & SJIF (Morocco) & $=5.667$ & OAJI (USA) & $=0.350$ \\
\hline
\end{tabular}

and economic indicators of the footwear enterprises in the regions of SFD and NCFD. the memory of V. A. Fokina is dedicated to: SB.nauch. articles and memories. Part 3. (pp.32-41). Moscow: MSUDT.

11. (2008). Quality management of competitive and demanded materials and products: Monograph / Yu. D. Mishin [etc.].; under the General editorship of doctor of technical
Sciences, prof. (Eds.). (p.654). Mine: Publishing house GOU VPO yurgues.

12. Prokhorov, V. T., et al. (2012). Managing production of competitive products in demand. under the General editorship of doctor of technical Sciences, Professor V. T. Prokhorov (Eds.). VPO yurgues. (p.280). Novocherkassk: yurgtu (NPI). 